\title{
Recurrent Novae in M31
}

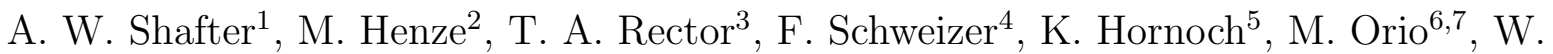 \\ Pietsch $^{8}$, M. J. Darnley ${ }^{9}$, S. C. Williams ${ }^{9}$, M. F. Bode ${ }^{9}$, J. Bryan ${ }^{10}$
}

\begin{abstract}
The reported positions of 964 suspected nova eruptions in M31 recorded through the end of calendar year 2013 have been compared in order to identify recurrent nova candidates. To pass the initial screen and qualify as a recurrent nova candidate two or more eruptions were required to be coincident within $0.1^{\prime}$, although this criterion was relaxed to $0.15^{\prime}$ for novae discovered on early photographic patrols. A total of 118 eruptions from 51 potential recurrent nova systems satisfied the screening criterion. To determine what fraction of these novae are indeed recurrent the original plates and published images of the relevant eruptions have been carefully compared. This procedure has resulted in the elimination of 27 of the 51 progenitor candidates (61 eruptions) from further consideration as recurrent novae, with another 8 systems (17 eruptions) deemed unlikely to be recurrent. Of the remaining 16 systems, 12 candidates (32 eruptions) were judged to be recurrent novae, with an additional 4 systems ( 8 eruptions) being possibly recurrent. It is estimated that $\sim 4 \%$ of the nova eruptions seen in M31 over the past century are associated with recurrent novae. A Monte Carlo analysis shows that the discovery efficiency for recurrent novae may be as low as $10 \%$

\footnotetext{
${ }^{1}$ Department of Astronomy, San Diego State University, San Diego, CA 92182, USA

${ }^{2}$ European Space Astronomy Centre, P.O. Box 78, 28692 Villanueva de la Cañada, Madrid, Spain

${ }^{3}$ Department of Physics and Astronomy, University of Alaska Anchorage, 3211 Providence Dr., Anchorage, AK 99508

${ }^{4}$ Carnegie Observatories, 813 Santa Barbara St., Pasadena, CA 91101, USA

${ }^{5}$ Astronomical Institute, Academy of Sciences, CZ-251 65 Ondrejov, Czech Republic

${ }^{6}$ Astronomical Observatory of Padova (INAF), 35122 Padova, Italy

${ }^{7}$ Department of Astronomy, University of Wisconsin, 475 N. Charter St., Madison, WI 53706, USA

${ }^{8}$ Max Planck Institute for extraterrestrial Physics, PO Box 1312, Giessenbachstr., 85741, Garching, Germany

${ }^{9}$ Astrophysics Research Institute, Liverpool John Moores University, Liverpool L3 5RF, UK

${ }^{10}$ McDonald Observatory, Austin, TX, 78712, USA
} 
that for novae in general, suggesting that as many as one in three nova eruptions observed in M31 arise from progenitor systems having recurrence times $\$ 100 \mathrm{yr}$. For plausible system parameters, it appears unlikely that recurrent novae can provide a significant channel for the production of Type Ia supernovae.

Subject headings: galaxies: stellar content - galaxies: individual (M31) — stars: novae, cataclysmic variables

\section{Introduction}

Classical novae are all semi-detached binary systems consisting of a late-type star that fills its Roche lobe and transfers material to a white dwarf companion (Warner 2008). The transferred material slowly accumulates on the white dwarf's surface until the temperature and density at the base of the accreted layer become sufficiently high for a thermonuclear runaway (TNR) to ensue, leading to a nova eruption (Starrfield et al. 2008, and references therein). The progenitor binary is not disrupted in the process, and following the eruption, the mass transfer process resumes, eventually leading to another eruption. The time interval between successive eruptions varies considerably depending on properties of the progenitor system such as the mass and temperature of the white dwarf component and the rate of accretion onto its surface.

Models of nova eruptions suggest that systems with massive and luminous (hot) white dwarfs can trigger a TNR after accreting a relatively small envelope. Thus, for a given accretion rate, such systems are expected to have the shortest recurrence times (e.g., Yaron et al. 2005: Townsley \& Bildsten 2005; Kato et al. 2014). Systems that have been observed to have more than one eruption (with recurrence times $\$ 100$ yr) have been traditionally referred to as "Recurrent Novae" (RNe), although the distinction with Classical Novae (CNe) is arbitrary given that strictly speaking all CNe are believed to be recurrent.

Given that RNe are believed to harbor the most massive white dwarfs among all nova binaries, they are of particular interest as potential Type Ia supernova (SNe Ia) progenitors (e.g., Maoz \& Mannucci 2012). In particular, in the so called "single degenerate" progenitor model, SNe Ia are produced when a massive white dwarf in a close binary system accretes material from its companion star, pushing it over the Chandrashekhar limit. If the white dwarf has a Carbon-Oxygen (CO) composition, the result is the explosive burning of the CO white dwarf (a deflagration) leading to a Type Ia supernova explosion. If, on the other hand, the white dwarf is a ONeMg core, the result is believed to be an accretion induced collapse leading to the production of a neutron star. 
Della Valle \& Livio (1996) estimated the frequency of RN outbursts relative to CN outbursts in the Galaxy, the LMC and in M31 and concluded that RNe were not a major channel for the production of SNe Ia. Here, we present a comprehensive study of the positions of the 964 nova eruptions reported in M31 from September 1909 to the end of 2013 with the goal of determining the fraction of nova eruptions that are recurrent, and thus associated with the massive white dwarf progenitors potentially capable of producing Type Ia supernovae.

\section{Identification of M31 Recurrent Nova Candidates}

In principle, searching for RN systems should be straightforward. One simply looks for spatial coincidences among the reported positions of nova candidates observed in M31. The database assembled by W. Pietsch ${ }^{1}$ is ideal for this purpose. In practice, however, uncertainties in the reported nova positions significantly complicate the process. The earliest recorded novae in M31 were discovered on photographic plates and reported primarily by Hubble (1929), Arp (1956), Rosino (1964, 1973), and Rosino et al. (1989). The equatorial coordinates were not reported, but instead Cartesian offsets from the nucleus were given in a system with the $\mathrm{X}$-axis oriented along the major axis of the galaxy, with $+\mathrm{X}$ pointing to the North-East. The $\mathrm{Y}$ axis passes through the nucleus, with $+\mathrm{Y}$ pointing to the North West (+Y points South-East in Arp's convention). Most of these early positions were reported to a precision of only $0.1^{\prime}$.

In order to account for differences of precision in the reported positions of novae, we have adopted an initial screening criterion that varies depending on the expected uncertainty of the nova position. In all cases, to minimize the chance of missing a potential RN, loose screening criteria have been adopted in the initial search. For CCD surveys conducted after 1980, where the coordinates are generally well determined, novae with reported positions that differed by as much as $0.1^{\prime}$ were considered to be potential RNe. We have also adopted this screening criterion for novae discovered in the photographic surveys of Rosino (1964, 1973); Rosino et al. (1989) where revised astrometry is now available (Pietsch et al., in preparation). To account for uncertainty in the published positions of novae from earlier photographic patrols (e.g. Hubble 1929, Arp 1956), where the reported nova positions are known to be less reliable, we have expanded our initial screening for RNe to include novae with reported positions that differed by as much as $0.15^{\prime}$.

Clearly, such a coarse screening process will introduce significant numbers of chance positional near-coincidences, or "false positives" in our search for RNe. We can gain some

${ }^{1}$ http://www.mpe.mpg.de/ m31novae/opt/m31/index.php 
insight into how likely chance positional coincidences are to affect our screening process by considering a "nearest neighbor nova" distribution. Figure 1 shows the result of binning the angular separations between each of the nova eruptions reported through the end of calendar year 2013 and their closest neighbor nova. The plot, which has been truncated to a maximum separation of $60^{\prime \prime}$, reveals that there are a total of 55 nova candidates with a neighbor within $0.1^{\prime}$. All of these novae are included within our coarse screen. An additional 8 novae from early photographic surveys have been included in the expanded screen.

In an attempt to quantify the significance of a given positional near-coincidence, we have estimated the probability of its occurrence as a function of position in M31. Specifically, for a given observed angular separation, $s$, the probability of a chance positional near-coincidence, $P_{C}$, can be estimated by considering the observed nova surface density (assumed to follow the background $R$-band light of the galaxy ${ }^{2}$ in the vicinity of the nova, which we estimate by considering the number of novae in an elliptical annulus centered on the nucleus of M31 that contains the position of the nova. The width of the annulus is taken to be $1^{\prime}$, with the inner and outer elliptical annuli located $0.5^{\prime}$ on either side of the nova's position. Our estimate of the surface density is then simply given by the number of novae observed in the annulus, $N$, divided by the area of the annulus, $A$. Since we do not know a priori which novae will be RN candidates, the computation of $P_{C}$ must consider the probability that any nova in the annulus lies within a distance $s$ of any other nova in the annulus. In practice, $P_{C}$, for a given nova pair is simply a function of the observed separation, $s$, and the surface density of novae at the location of interest. Specifically, we have

$$
P_{C} \simeq 1-\prod_{k=1}^{N-1}(1-k x)
$$

where $x=\pi s^{2} / A$ is the overlap area for a chance positional coincidence of separation less than or equal to $s$. Not surprisingly, the probability of a chance positional near-coincidence is particularly high close to the center of M31 where the nova density is the highest (e.g., Ciardullo et al. 1987).

Out of a total of 964 nova eruptions reported in M31 through the end of 2013 3 , a total of 118 RN candidates have survived our initial screening process and are presented in Table 1 .

\footnotetext{
${ }^{2}$ We ignore possible variations in the (recurrent) nova density between bulge and disk populations, which are assumed to be small.

${ }^{3}$ Of these, 25 nova candidates have been deemed to be non-novae. However, we have included all nova candidates in our screen as some of the systems, e.g. M31N 1957-10b, have possibly been misclassified. We discuss the nature of all objects that pass our screen in the following section.
} 
The first column gives the first recorded eruption of a $\mathrm{RN}$ candidate with possible subsequent eruptions given in column 2. The time interval between the initial outburst of a nova and its possible subsequent eruptions $(\Delta t)$, the reported spatial separation $(s)$, the isophotal radius $(a)$ of the nova (i.e., the semimajor axis of an elliptical isophote that passes through the position of the nova), the number of novae $(N)$ observed in the $1^{\prime}$ wide annulus passing through the position of the nova, and the probability of a chance positional coincidence $\left(P_{C}\right)$, computed from Equation (1), are also summarized in Table 1. For systems with multiple recurrences, we also include screening data between all subsequent recurrences.

\section{Individual Systems}

Below we consider the individual RN candidates that have passed our initial screen. For all RN candidates, we attempted to locate the original plate or digital image data in order to make a detailed comparison of the nova positions. We were largely successful, with the exception of some images taken as part of the Ciardullo et al. (1987) survey that are no longer available. Photographic plates, for example from the surveys of Hubble (1929) and $\operatorname{Arp}(1956)$, were scanned and converted into FITS images for comparison with modern CCD images. Images of the eruptions of a given RN candidate have been carefully registered using the geomap and geotran routines in $\mathrm{IRAF}_{4}^{4}$. Once aligned, the relative spatial positions of the novae have been displayed through a comparison image formed by the ratio of the images of the individual eruptions.

In cases where the positions of the original nova eruption appears to coincide within observational errors with the subsequent eruption(s), we have re-computed the astrometry and determined revised coordinates for objects with poorly determined positions (e.g., those systems discovered on photographic plates prior to $\sim 1980$ ). Our revised coordinates are given in Table 2. For RN candidates that erupted in the early part of the 20th century (e.g., novae from the Hubble survey), there can be significant proper motion of the field stars used in the revised astrometry. When known, we have excluded any field stars with measured proper motions in excess of $10 \mathrm{mas} / \mathrm{yr}$ in our astrometric solutions. In most cases we estimate our revised astrometry to produce nova positions accurate to $1^{\prime \prime}$.

Given the limitations of the data (e.g., finite temporal and spatial resolution of the images), it is never possible to establish with absolute certainty that a given candidate is in fact a RN system. For example, there is always the possibility of a chance positional

\footnotetext{
${ }^{4}$ IRAF is distributed by the National Optical Astronomy Observatory, which is operated by the Association for Research in Astronomy, Inc. under cooperative agreement with the National Science Foundation.
} 
coincidence within observational uncertainty of two (or more) unrelated nova eruptions. In other cases, given limited temporal sampling, there is the possibility for long period variable (LPV) stars to masquerade as novae (e.g., Shafter et al. 2008). Despite these challenges, in the discussion to follow we have brought to bear all available evidence in judging the likelihood that a given candidate is a RN. This process has led us to place the RN candidates from our initial screen into one of four categories: (1) Recurrent Novae systems where the weight of the evidence strongly supports the spatial coincidence of the eruptions, and where the probability of a chance positional coincidence is low $\left(P_{C} \lesssim 0.1\right),(2)$ Possible Recurrent Novae - systems where there remains some doubt as to the precise spatial coincidence of the eruptions or where the nature of the object is in doubt (e.g., a possible LPV or foreground Galactic dwarf nova), (3) Unlikely Recurrent Novae - often systems without available finding charts where the probability of chance positional near coincidence is relatively high $\left(P_{C} \gtrsim 0.4\right)$, and (4) Rejected Recurrent Nova Candidates - systems where the archival images clearly establish that the eruptions are not spatially coincident. We begin by discussing the systems judged to be RNe.

\subsection{Recurrent Novae}

\subsubsection{M31N 1919-09a}

M31N 1998-06a was discovered at $m_{H \alpha}=16.3$ on 1998 June 06 UT as part of the Kitt Peak National Observatory's Research-Based Science Education (RBSE) program (Rector et al. 1999). The object lies approximately $10.5^{\prime}$ from the nucleus of M31 and just $1.8^{\prime \prime}$ from the nominal position of M31N 1919-09a (Hubble 1929). The probability of a chance positional coincidence with $s \leq 1.8^{\prime \prime}$ at this location is estimated to be 0.030 , making the object a strong RN candidate. To confirm this possibility we have located the original discovery plate (\#5054) in the Carnegie archives. The plate was taken on 1919 September 21, and shows the nova at $m_{p g}=17.6$. Figure 2 shows a scan of the plate compared with the RBSE image of 1998-06a. The novae clearly appear to be spatially coincident. Revised astrometry of M31N 1919-09a (see Table 2 ) shows that the nova lies $\sim 1.5^{\prime \prime}$ from the measured position of 199806a, dropping the probability of a chance coincidence to 0.016. To within the uncertainty of the measured positions, the novae are consistent with being spatially coincident, and we conclude that M31N 1919-09a is very likely a RN. We note that M31N 1998-06a was detected as a faint supersoft X-ray source (SSS) $1028 \pm 92$ days after the optical outburst (Pietsch et al. 2005). Owing to the low number of detected photons, no estimate of the effective temperature was possible. The SSS disappeared $1773 \pm 463$ days after outburst (Pietsch et al. 2007a). 


\subsubsection{M31N 1923-12c}

M31N 2012-01b was a rapidly-fading, He/N nova that was discovered on 2012 January 21.419 UT by K. Nishiyama and F. Kabashima approximately 6 " from the nominal position of M31N 1923-12c, a nova discovered by Hubble on 1923 December 11. We located Hubble's plate $(\mathrm{H} 348 \mathrm{H})$ in the Carnegie archives and produced a scan of the plate, a portion of which is reproduced in Figure 3 along with a chart of 2012-01b courtesy of K. Nishiyama and F. Kabashima (Miyaki-Argenteus Observatory, Japan). Nova 1923-12c, which is indicated by Hubble's hand-drawn ink marks, appears to lie at the same position as 2012-01b. The revised astrometry of 1923-12c (see Table 2) confirms that the nova lies within $0.45^{\prime \prime}$ of 2012-01b. The probability of a chance coincidence at this location in the galaxy is estimated to be just 0.0033. We conclude, as initially reported in Shafter et al. (2012b), that 1923-12c is a RN, and that 2012-01b is a subsequent eruption of Hubble's 1923 nova.

\subsubsection{M31N 1926-06a}

A possible recurrence of M31N 1926-06a, 1962-11a, was discovered by Rosino (1964) as part of his multi-year nova survey. As pointed out by Henze et al. (2008a), the recurrence was discovered independently by Börngen (1968). Our expanded screen shows that the published position of M31N 1962-11a lies 6.3" from the reported position of 1926-06a (nova \#62 in Hubble (1929)). We were successful in locating the original discovery plates (H304D and H309D) in the Carnegie archives. Figure 4 shows a comparison of the position of M31N 192606a and that of 1962-11a from Henze et al. (2008a). The novae appear coincident. Revised astrometry of M31N 1926-06a (see Table 2 reveals that Hubble's nova lies within 1.40" of the position of 1962-11a. The probability of a chance coincidence at this location in the galaxy is just 0.010 , and we conclude that M31N 1962-11a is almost certainly a recurrence of 1926-06a.

\subsubsection{M31N 1926-0\%c}

Our coarse screen indicated that M31N 1926-07c, nova \#65 in Hubble (1929), might be coincident with a nova that erupted 54 years later, M31N 1980-09d (Rosino et al. 1989). Examination of the finding charts for the two novae shown in Figure 5, clearly demonstrate that the novae are not spatially coincident. However, revised astrometry for M31N 1926-07c (see Table 2) and further analysis of it's precise position unexpectedly revealed that the nova was coincident with both M31N 1997-10f and 2008-08b, a nova pair also identified as a RN 
candidate in our coarse screen.

M31N 1997-10f was discovered by Shafter \& Irby (2001), and confirmed by Lee at al. (2012). A possible recurrence was discovered by Henze et al. (2008a), who noted that the position of M31N 2008-08b was nearly coincident with 1997-10f ( $\left.P_{C} \simeq 0.045\right)$, making the latter object likely a RN. We have located the original image from Shafter \& Irby (2001) and re-measured the coordinates of M31N 1997-10f (see Table 2), confirming the association with 2008-08b.

The positions of M31N 1926-07c, 1997-10f and 2008-08b are compared in Figure 6 , which shows that all 3 novae are indeed spatially coincident to within the resolution of the images $\left(P_{C} \simeq 0.024\right)$. We also note that M31N 2008-08b was spectroscopically classified as a possible $\mathrm{He} / \mathrm{N}$ nova (with a quite narrow $\mathrm{H} \alpha \mathrm{FWHM}$ ) by di Mille et al. (2008). We conclude that M31N 1926-07c is a RN with 1997-10f and 2008-08b representing subsequent outbursts.

\subsubsection{M31N 1945-09c}

M31N 1975-11a was observed to erupt in the outskirts of M31 at an isophotal radius, $a \sim 29^{\prime}$, and less than $1^{\prime \prime}$ from the reported position of 1945-09c (Baade \& Arp 1964). At this position in the galaxy, the probability of a chance positional coincidence is negligible $(\sim 0.0004)$. We conclude, as did Henze et al. (2008a), that M31N 1945-09c is a RN. Finding charts for the novae are compared in Figure 7 , and confirm that the novae are indeed spatially coincident. The revised coordinates for M31N 1945-09c are given in Table 2 lie only $0.41^{\prime \prime}$ from the position of 1975-11a leading to an even smaller probability of a chance positional near coincidence, $P_{C} \simeq 0.0001$.

\subsubsection{M31N 1960-12a}

M31N 1960-12a erupted relatively close to the nucleus of M31 $\left(a \sim 4.4^{\prime}\right)$ and our initial screen revealed two possible recurrences: $1962-11 \mathrm{~b}$ and 2013-05b. Figure 8 shows the position of M31N 1960-12a compared with 2013-05b. Revised astrometry of M31N 1960-12a yields the coordinates given in Table 2, and shows that the nova is coincident with the published position of 2013-05b to within $\sim 0.9^{\prime \prime}$. We estimate the probability of a chance coincidence at this location in M31 is $\sim 0.091$, and thus we consider it very likely that M31N 2013-05b is a recurrence of 1960-12a.

As will be discussed further below, M31N 1960-12a, 1962-11b, and 2013-05b were also flagged as a possible recurrences of 1953-11a. Of these, M31N 1962-11b was the most likely 
recurrence with a reported separation $s \sim 2.9^{\prime \prime}$. A comparison of the finding charts shown in both Figures 8 and later in 27 reveals that none of these novae are coincident with M31N 1953-11a.

\subsubsection{M31N 1963-09c}

M31N 1963-09c, which was discovered by Rosino (1973), has been observed to have three possible recurrences. 1968-09a (Rosino 1973), 2001-07b (Lee at al. 2012), and 2010-10e (Hornoch et al. 2010) have all erupted within 1.32" of the reported position of 1963-09c. Rosino (1973) was first to note that M31N 1963-09c and 1968-09a appeared to be spatially coincident. Given that the novae are located rather far $\left(a \sim 17.6^{\prime}\right)$ from the nucleus of M31, the probability of a chance positional coincidence with $s<1.32^{\prime \prime}$ is $\sim 0.01$. Finding charts for the eruptions of M31N 1963-09c and 1968-09a from the survey of Rosino (1973) are compared with the chart of 2010-10e (Hornoch et al. 2010) in Figure 9. The novae are clearly coincident, and we conclude, as did Shafter et al. (2010), that M31N 1963-09c is a RN in M31. Consistent with this finding, spectroscopic observations of the most recent eruption (2010-10e) by Shafter et al. (2010) revealed that the nova was a member of the He/N class. In addition, M31N 2010-10e was first detected as a bright SSS only $14 \pm 1$ days after outburst by Pietsch et al. (2010). Henze et al. (2014a) described an X-ray spectrum characterized by a relatively high effective (blackbody) temperature of $61_{-3}^{+6} \mathrm{eV}$ and reported that the SSS had disappeared on day $92 \pm 5$ after the optical outburst. Additionally, during one of the observations of Henze et al. (2014a) the X-ray light curve of M31N 2010-10e showed strong, aperiodic variability on time scales of hours.

This RN is noteworthy in that the first two recorded eruptions occurred within a time span of just 5 years. This was the shortest recurrence time measured for any RN until the discovery that M31N 2008-12a has a recurrence time of just 1 year (see below). Williams et al. (2014) has studied the field of M31N 2010-10e in search of a potential red giant companion for the nova. Archival Hubble Space Telescope (HST) images reveal a resolved source $0.80 \sigma$ away from the nominal position of the nova. According to Williams et al. (2014), the density of stars at this location suggests there is a $15.6 \%$ probability of such an alignment occurring by chance. 


\subsubsection{M31N 1966-09e}

M31N 1966-09e, discovered as nova \#71 by Henze et al. (2008a) on archival Tautenburg Schmidt plates, is noteworthy in that it was observed to erupt in the outskirts of M31 (almost a full degree from the nucleus of the galaxy). A recurrence was observed as M31N 2007-08d (Pietsch et al. 2007b). The reported positions differ by just $0.36^{\prime \prime}$, and at this position in the galaxy the probability of a chance coincidence is negligible. Astrometry of M31N 1966-09e yields the coordinates given in Table 2. Figure 10 confirms that the two eruptions are indeed spatially coincident, and we conclude that M31N 1966-09e is a RN. The spectroscopic type and light curve properties of M31N 2007-08d was measured by Shafter et al. (2011b) as a relatively slow $\left(t_{2} \sim 81 \pm 11 \mathrm{~d}\right)$ Fe II system. The recurrence was also observed in the infrared by the Spitzer Space Telescope but did not reveal an infrared excess that could be attributed to dust formation (Shafter et al. 2011a).

\subsubsection{M31N 1982-08b}

M31N 1982-08b was discovered in the outskirts of M31 as nova \#27 in the survey of Sharov \& Alksnis (1992). A likely recurrence (nominally located $\sim 3^{\prime \prime}$ away) was discovered by Shafter \& Irby (2001), who reported a nova discovered on 1996 August 12. Subsequently, the nova acquired the designation M31N 1996-08c. In reviewing the original data to produce a finding chart, we have determined that the nova was actually discovered a year later, on 1997 August 01 UT. Revised astrometry given in Table 2 shows that the novae are spatially coincident to less than $1^{\prime \prime}$, which is less than the uncertainty in the absolute positions. Finding charts for the images are shown in Figure11, confirming that the novae are spatially coincident. The probability of a chance positional coincidence is negligible $\left(P_{C} \simeq 0.0001\right)$, and we conclude that M31N 1982-08b is recurrent.

\subsubsection{M31N 1984-07a}

M31N 1984-07a was discovered by Rosino et al. (1989) very close to the nucleus of M31. There have been several subsequent novae seen to erupt within 2" of M31N 1984-07a, including 2001-10c, 2004-02a, 2004-11f, and 2012-09a. We have remeasured the position of M31N 1984-07a using the image from the survey of Rosino et al. (1989), and determined the revised coordinates given in Table 2. The revised coordinates are within 1.1", 2.3", 0.3", and $0.6^{\prime \prime}$ of M31N 2001-10c, 2004-02a, 2004-11f, and 2012-09a, respectively. Thus, it appears likely that M31N 2004-11f and 2012-09a are recurrences, with 2001-10c and 2004-02a being 
less likely to be recurrences. Pietsch et al. (2007a) have studied the positions of M31N 2001-10c, 2004-02a, and 2004-11f, and concluded that these three novae are not spatially coincident. They were not able, however, to rule out the possibility that M31N 2004-11f was a recurrence of 1984-07a. The positions of M31N 1984-07a, 2004-11f, and 2012-09a are shown in Figure 12. It appears very likely that both M31N 2004-11f and 2012-09a are recurrences of 1984-07a.

M31N 2004-11f was detected as a bright and fast SSS by Pietsch et al. (2007a). The X-ray emission was already present 34 days after the optical outburst and had started to decline in luminosity soon afterwards, by day 55 after outburst. Pietsch et al. (2007a) reported strong indications that the X-ray emission was supersoft, but unfortunately did not detect sufficient photons to estimate the effective SSS temperature. Notably, 10 months prior to the discovery of M31N 2004-11f, Pietsch et al. (2007a) identified what they believed to be the "pre-nova" in archival HST images. If so, and if M31N 2012-09a is also a recurrence of 1984-07a, it is noteworthy that archival HST images of the field of 2012-09a were analyzed by Williams et al. (2014) who found no compelling evidence for a red giant companion.

\subsubsection{M31N 199\%-11k}

M31N 1997-11k was discovered as part of the RBSE program (Rector et al. 1999) and confirmed by Lee at al. (2012). Two likely recurrences have been observed: M31N 2001-12b (Lee at al. 2012) and 2009-11b (Henze et al. 2009b). Henze et al. (2009b) discussed the possibility that M31N 1997-11k might either be a RN in M31 or a dwarf nova in the Galaxy, and urged spectroscopic observations of 2009-11b to resolve the issue. Kasliwal et al. (2009) obtained a spectrum clearly establishing that M31N 2009-11b was an Fe II class nova in M31. Finding charts for the three objects from the RBSE program are shown in Figure 13. We conclude, in agreement with earlier suggestions, that M31N 1997-11k is a RN. Given the relatively short recurrence time, it would appear that the mass accretion rate in this system must be relatively high. Williams et al. (2014) ruled out the presence of a luminous red giant secondary in the progenitor system of M31N 2009-11b.

\subsubsection{2. $M 31 N 2008-12 a$}

M31N 2008-12a, which was discovered by K. Nishiyama and F. Kabashima (MiyakiArgenteus Observatory, Japan), was first seen to erupt in the outskirts of M31 $\left(a \simeq 49^{\prime}\right)$. The nova has subsequently been seen to have had four additional outbursts: M31N 2009- 
12b (Tang et al. 2014), 2011-10e (Barsukova et al. 2011), 2012-10a (Shafter 2012a), and 2013-11f (Tang et al. 2013), all 4 occurring within $1^{\prime \prime}$ of the measured position of 2008$122^{5}$. At this relatively large galactocentric radius, the probability of a chance positional coincidence is negligible, and it seems clear that the novae represent brightenings of the same progenitor system (see Fig. 14). It would appear that M31N 2008-12a must be either a RN with an extremely short interval between eruptions ( 1 yr), or that the 2009, 2011, 2012 and 2013 events are simply rebrightenings of an unusually slow nova. Recent observations have shown that the latter possibility is untenable. In particular, the He/N (Shafter 2012a) spectroscopic type along with the super-soft X-ray behavior strongly suggests that the object is in fact a short recurrence time RN (Darnley et al. 2014a; Henze et al. 2014b; Tang et al. 2014). Further, as shown by Darnley et al. (2014a), the spectral energy distribution of the quiescent counterpart of M31N 2008-12a is consistent with a bright accretion disk (and thus a high accretion rate) in this system.

A literature search has revealed three additional X-ray detections of M31N 2008-12a in February 1992 and January 1993 (White et al. 1995) as well as in September 2001 (Williams et al. 2004). The super-soft X-ray properties of the two earlier outbursts (short duration, relatively high effective temperature) are in agreement with the 2013 X-ray detection (Henze et al. 2014b). These authors reported a very short SSS phase, appearing on day $6 \pm 1$ and disappearing on day $19 \pm 1$ after the optical outburst, with an exceptionally high effective (blackbody) temperature of $97_{-4}^{+5} \mathrm{eV}$. With eight recorded outbursts, M31N 2008-12a is a very unusual object that merits continued attention. An archival study on potential previous outbursts is in preparation (Henze et al. 2015).

\subsection{Possible Recurrent Novae}

\subsubsection{M31N 1953-09b}

M31N 1953-09b was discovered by Arp (1956) as part of his classic M31 nova study (Arp \#3). A subsequent nova, M31N 2004-08a, discovered by K. Hornoch (Pietsch et al. 2007a), was seen to erupt $\sim 4.8^{\prime \prime}$ away from the reported position of 1953-09b. At the location of the nova $\left(a \sim 5.8^{\prime}\right)$, the probability of a chance coincidence is quite high $\left(P_{C} \simeq 0.640\right)$. Nevertheless, we have located the original plate from Arp's survey (S967A) and compared the position of M31N 1953-09b with that of 2004-08a in Figure 15. A careful inspection of

5 While this paper was under review another outburst of M31N 2008-12a, designated 2014-10c, was discovered on 2014 Oct 02.903 UT (Darnley et al. 2014b). 
the charts shows that M31N 1953-09b may be just slightly south of the position of 2004-08a, but to within the limits of the seeing disks $\left(\sim 1.5^{\prime \prime}\right)$, it is possible that the novae could be

spatially coincident. Revised coordinates for M31N 1953-09b are given in Table 2, lowering the probability of a chance positional coincidence to $P_{C} \simeq 0.131$.

It is worth noting that Lee at al. (2012) judge the light curve of M31N 2004-08a to be questionable for a nova, so there remains some doubt regarding the nature of this object. That said, M31N 2004-08a was detected as a SSS by Pietsch et al. (2007a). The nova showed a very short SSS phase and was only detected around day 60 after outburst without being visible about 30 days before and after. Pietsch et al. (2007a) also estimated a high effective (blackbody) temperature of around $80 \mathrm{eV}$. In view of the available evidence, the nature of M31N 1953-09b remains uncertain, with the possibility that the object is a $\mathrm{RN}$ remaining viable.

\subsubsection{M31N 1961-11a}

M31N 1961-11a erupted near the nucleus of M31 $\left(a \sim 3^{\prime}\right)$ and was discovered as nova \#35 in the survey of Rosino (1973). A possible recurrence, M31N 2005-06c, discovered by K. Hornoch (Pietsch et al. 2007a), erupted $\sim 2.4^{\prime \prime}$ from the reported position of 1961-11a and thus passed our initial RN screening. Given the nominal separation of the two novae, and their close proximity to the nucleus, the probability of a chance positional coincidence is quite high $\left(P_{C} \simeq 0.691\right)$. However, a careful comparison of the finding charts for the two novae shown in Figure 16 reveals that they are in fact spatially coincident to within measurement uncertainties. Revised astrometry of M31N 1961-11a based on the published chart (see Table 2) shows that the two novae appear to be separated by just $0.45^{\prime \prime}$, with a probability of a chance coincidence, $P_{C} \simeq 0.042$. Despite the spatial coincidence, Lee at al. (2012) have identified a variable star near the position of M31N 2005-06c, and have called into question the nature of the object. In view of their findings, we hesitate to definitively classify the object as a RN, and have instead included it in our list of uncertain RNe.

\subsection{3. $M 31 N 1966-08 a$}

M31N 1966-08a and 1968-10c were discovered as novae \#66 and \#81 in the survey of Rosino (1973), who gives the coordinates of the novae as being identical. The object is located at a relatively large galactocentric radius $\left(a \simeq 31.2^{\prime}\right)$ and there is very little chance that the objects could be distinct systems. The positions of the two novae are shown in 
Figure 17, confirming that the objects are in fact spatially coincident. Sharov \& Alksnis (1989) argue that given the short interval between eruptions, the system is likely a Galactic foreground U Gem star (dwarf nova). That said, the possibility that the object is a RN with a very short recurrence time cannot be ruled out, and we have classified the object as an uncertain RN. Whether or not the system is a short recurrence time RN or a Galactic dwarf nova, it is quite surprising that more eruptions of the star have not been observed!

\subsubsection{M31N 1990-10a}

M31N 1990-10a was discovered on 1990 October 13.16 UT during a routine photographic patrol of M31 (Bryan 1990). Our initial screen reveals two possible recurrences. The first, M31N 1997-10b, was a faint nova candidate reported by Shafter \& Irby (2001). A reexamination of the original image shows that the object was likely a CCD artifact, and not a nova, as noted by Lee at al. (2012). Another possible recurrence was M31N 200707a (Hatzidimitriou et al. 2007a), which erupted $\sim 0.8^{\prime \prime}$ away from the reported position of 1990-10a. Revised astrometry of M31N 1990-10a (see Table 2) reveals that the two novae are spatially coincident to within $0.59^{\prime \prime}\left(P_{C} \simeq 0.041\right)$. However, as can be seen in Figure 18, it appears that M31N 2007-07a may lie just slightly to the NW of 1990-10a. The relatively poor seeing of the M31N 1990-10a discovery image makes it impossible to establish conclusively whether or not the two novae are in fact coincident. We conclude that M31N 1990-10a is a possible RN.

\subsection{Unlikely Recurrent Novae}

\subsubsection{M31N 1932-09d}

M31N 1932-09d was discovered by Stratton (1936) and lies $\sim 3.6^{\prime \prime}$ from the position of a more recent nova, 2001-07d, which was discovered by Li (2001) and independently by the Wendelstein Calar Alto Pixellensing Project (Lee at al. 2012) and in data from the POINTAGAPE survey (Darnley et al. 2004). Unfortunately, we have not been able to locate a finding chart for M31N 1932-09d, so we are unable to definitely establish whether or not the object is a RN. We note that eruptions with a nominal separation of $3.6^{\prime \prime}$ or less at a projected distance of $\sim 3^{\prime}$ from the nucleus of M31 have probability of a chance coincidence that is quite high $\left(P_{C} \simeq 0.939\right)$. We conclude that M31N 2001-07d is unlikely to be a recurrence of 1932-09d. 


\subsubsection{M31N 195\%-10b}

As noted by Alksnis \& Zharova (2000), M31N 1957-10b (PT And) has exhibited several recurrences $(1983,1986,1988$, and 1998) over the past 30 years in addition to its most recent outburst in 2010, M31N 2010-12a (Ruan \& Gao 2010a; Zheng et al. 1997). Sharov \& Alksnis (1989) and Alksnis \& Zharova (2000) have argued based on its light curve properties that PT And is a Galactic dwarf nova system, while Cao et al. (2012) suggest based on an optical spectrum that the object may indeed be a RN in M31. We have not shown finding charts for the outbursts of PT And because it is clear that they are spatially coincident and the light curves are consistent between eruptions (Alksnis \& Zharova 2000). The only question is whether the object is a RN in M31 or a Galactic dwarf nova. After reviewing available data, including a spectrum published online by $\mathrm{Ka}^{6}$, which shows a blue continuum with no prominent emission features, we conclude that the object is most likely a foreground Galactic dwarf nova and not a RN.

\subsubsection{M31N 1982-09a}

M31N 1982-09a is the first of 6 novae to be discussed below that were all discovered as part of the Ciardullo et al. (1987) $\mathrm{H} \alpha$ survey for novae in M31. Unfortunately, the original CCD data for these novae have been lost, making it impossible to create the finding charts necessary to definitively test whether these novae are recurrent. Most of these novae erupted quite close to the nucleus of M31 and their putative recurrences have high probabilities of chance positional coincidence.

M31N 1982-09a was one of the Ciardullo et al. (1987) novae that erupted very close $\left(a \sim 1.08^{\prime}\right)$ to the nucleus of M31. A possible recurrence, M31N 2011-02b, was seen to erupt $\sim 5.46^{\prime \prime}$ from the reported position of 1982-09a. The estimated probability of a chance positional coincidence is extremely high, with $P_{C} \simeq 0.998$. Despite the fact that a direct comparison of the finding charts is not possible, the coordinates are unlikely to be in error by as much as 5 " making it is extremely unlikely that M31N 2011-02b is a recurrence of 1982-09a.

6 http://www.cc.kyoto-su.ac.jp/ kao/blog/index.php/view/20 


\subsubsection{M31N 1983-09c}

M31N 1983-09c was also discovered as part of the Ciardullo et al. (1987) nova survey. A possible recurrence, M31N 1997-11c (Rector et al. 1999), erupted $\sim 6^{\prime \prime}$ from the reported position of 1983-09c, and thus just passed our RN screening criterion. The novae erupted with $a \sim 8^{\prime}$ from the nucleus, and probability of a chance positional coincidence is estimated to be $\sim 0.5$. Since a finding chart for M31N 1983-09c is not available, we cannot test whether these novae might be spatially coincident. However, given that both novae were registered with CCD detectors and their positions computed directly from the images, it is highly unlikely that a positional error of $\sim 6^{\prime \prime}$ would be possible if M31N 1997-11c was a recurrence of 1983-09c.

\subsubsection{M31N 1984-096}

M31N 1984-09b was another nova found in the survey by Ciardullo et al. (1987), and like 1982-09a quite close to the nucleus of the galaxy $\left(a \simeq 1.27^{\prime}\right)$. A possible recurrence, M31N 2012-12a, was flagged by our screen. The latter nova was discovered by Hornoch \& Galad (2012), and found to be a member of the Fe II spectroscopic class by Shafter et al. (2012b). Despite the fact that M31N 2012-12a has a reported position that is only $1.5^{\prime \prime}$ from that of 1984-09b, the probability of a chance positional coincidence so close to the nucleus of M31 is relatively high $\left(P_{C} \simeq 0.450\right)$. Unfortunately, since a finding chart for M31N 1984-09b no longer exists, once again we cannot evaluate whether the objects are spatially coincident. Williams et al. (2014) studied the field of M31N 2012-12a and found that there is a source within $3 \sigma\left(1.532 \mathrm{ACS} / \mathrm{WFC}\right.$ pixels or $\left.0.077^{\prime \prime}\right)$ of the nova's position. According to Williams

et al. (2014), the local stellar density suggests a probability of chance coincidence with this separation is $19.9 \%$, and it is unclear whether the source might be associated with the nova progenitor.

\subsubsection{M31N 1985-09d}

As with two of the three the previous Ciardullo et al. (1987) novae, M31N 1985-09d was discovered close to the nucleus of M31 $\left(a \simeq 0.81^{\prime}\right)$. A possible recurrence, M31N 2009-08d, located $\sim 4.12^{\prime \prime}$ away was discovered by K. Hornoch (Henze et al. 2009a). Despite the fact that without a finding chart we cannot definitively rule out the possibility that M31N 198509d is recurrent, it seems highly unlikely given that the coordinates differ by more than $4^{\prime \prime}$ and the probability of a chance positional coincidence with this separation at this location in 
the galaxy is very high $\left(P_{C} \simeq 0.993\right)$. We note that another nova, M31N 2005-05b was also flagged by our screen, and lies $4.6^{\prime \prime}$ away from the nominal position of 2009-08d. As shown below M31N 2005-05b and 2009-08d are not spatially coincident. Given that the reported position of M31N 1985-09d is even further from 2005-05b than it is from 2009-08d, it is even less likely that 2005-05b could be a recurrence of 1985-09d. Finally, we note that M31N 2009-08d was determined to be an Fe II nova by di Mille et al. (2009), and no evidence for a red giant companion often associated with RNe was found in the search for M31 nova progenitors by Williams et al. (2014).

\subsubsection{M31N 1985-10c}

M31N 1985-10c, another nova discovered by Ciardullo et al. (1987), was again found extremely close to the nucleus of M31 $\left(a \simeq 0.30^{\prime}\right)$. Two possible recurrences have been subsequently noted, M31N 1995-12a and 2003-10b. In addition to lacking a finding chart for M31N 1985-10c, we were also unable to locate a chart for M31N 1995-12a despite reexamining an image from the survey of Shafter \& Irby (2001) taken on 1996 January 14, 27 days after the nova was discovered by Ansari et al. (2004). A chart does exits for M31N 2003-10b (Fiaschi et al. 2003), but given the extremely close proximity to the nucleus, it is unlikely that finding charts, even if available for all three novae, would be useful in definitively

establishing the spatial coincidence of the objects. Based on the published coordinates, M31N 1995-12a and 2003-10b have probabilities of chance coincidence with 1985-10c of $P_{C} \simeq 0.708$ and $P_{C} \simeq 0.422$, respectively, while 1995-12a and 2003-10b have a probability of chance coincidence with respect to each other of $P_{C} \simeq 0.953$. We conclude that there is no compelling evidence that M31N 1985-10c is a RN.

\subsubsection{M31N 1986-09a}

M31N 1986-09a, the final nova from the Ciardullo et al. (1987) survey to be discussed, was also discovered relatively close to the nucleus of M31 $\left(a \simeq 1.63^{\prime}\right)$. A possible recurrence is M31N 2006-09b, which was seen to erupt $\sim 4.8^{\prime \prime}$ away. Despite the fact that a comparison of the finding charts for these novae is not possible, as with the previous two systems, it is unlikely that the coordinates would be in error by as much as $4.8^{\prime \prime}$ if the system was a RN. At this separation, the probability of a chance positional near coincidence, $P_{C} \simeq 0.994$. 


\subsection{Rejected Recurrent Nova Candidates}

\subsubsection{M31N 1909-09b}

The relatively recent nova M31N 2009-02b (Pietsch et al. 2009a) erupted $\sim 4.2^{\prime \prime}$ SSW of the nominal position of M31N 1909-09b. M31N 1909-09b is the second nova listed by Hubble (1929), and was discovered on the rise on 1909 September 12 on a plate taken by Ritchey using the Mount Wilson 60-in reflector (Ritchey 1917). The nova reached a maximum brightness of $m_{p g}=16.7$ on September 15, and faded slowly to $m_{p g}=18.0$ on November 07 . The available photometry places a lower limit on the $t_{2}$ time of at least 55 days, making M31N 1909-09b a very slow nova. A comparison of the position of M31N 1909-09b from plate S19-Ri (1.5 hr exposure) taken by Ritchey on 13 September 1909 and the position of 2009-02b from the SuperLOTIS project clearly shows that the two novae are in fact distinct objects separated by 10.5" (see Fig. 19). Astrometry of M31N 1909-09b based on a scan of the Ritchey plate yields the revised coordinates given in Table 2 .

\subsubsection{M31N 1918-02b}

Our screen has revealed that the recent Fe II nova M31N 2013-10g, which reached a peak brightness of $R=17.5$ (Fabrika et al. 2013), erupted within $\sim 12^{\prime \prime}$ of nova \#9 in Hubble's survey (Hubble 1929). The latter nova was discovered on 1918 February 10 UT (plate S162-Ri), and reached a similar peak brightness $\left(m_{p g}=17.5\right)$. Despite the relatively large nominal separation, given the uncertainty in the coordinates derived from these early photographic surveys, we took a closer look at the positions of these two novae. As can be seen in Figure 20, the novae clearly represent eruptions from distinct progenitors, and we conclude that M31N 2013-10g is not a recurrence of 1918-02b. Updated astrometry for M31N 1918-02b yields the revised coordinates given in Table 2.

\subsubsection{M31N 1923-02a}

M31N 1923-02a, nova \#22 in the survey of Hubble (1929), erupted close to the nucleus of M31 $\left(a \simeq 1.74^{\prime}\right)$. Since then, there have been three potential recurrences M31N 196712a (Rosino 1973), 1993-11c (Shafter \& Irby 2001) and 2013-08b (Hornoch \& Vrastil 2013). Finding charts for the novae are shown in Figure 21. It is clear that M31N 1923-02a, 1967$12 \mathrm{a}$ and $1993-11 \mathrm{c}$ are different novae. It is less obvious that M31N 2013-08b is distinct from 1967-12a, but the comparison image clearly shows that the novae are not spatially coincident. 
Revised astrometry of M31N 1923-02a and 1967-12a are given in Table 2, confirming that all four novae are distinct objects.

\subsubsection{M31N 1924-02b}

M31N 1924-02b was flagged as a potential RN candidate because nova 1995-09d was observed to lie $5.2^{\prime \prime}$ from its reported position. M31N 1924-02b was a faint $\left(m_{p g}=19\right)$ transient discovered on 1924 February 03 UT by Hubble (1929) who noted that the object varied between $m_{p g}=19.0$ and 19.5 for several years. Given its long-term photometric behavior, it seems clear that M31N 1924-02b is not a nova in M31, but likely a fainter long-period variable.

\subsubsection{M31N 1924-08a}

M31N 1987-12a was discovered at $m_{p g}=16.8$ on 1987 December 20.13 UT (Bryan 1987). Our screen revealed that the nova erupted 3.1" from the reported position of M31N 1924-08a. At an isophotal radius of just $a \sim 2.61^{\prime}$, the probability of a chance coincidence is quite high $\left(P_{C} \simeq 0.900\right)$. Fortunately, we were able to recover both the original 35-mm negative from the 1987 observation as well as the original Mt Wilson plates, H241D and H246D, from 1924 August 26 and 28, respectively. Figure 22 shows the field of M31N 1924-08a taken from plate H246D compared with the position of $1987-12$ a from a $35 \mathrm{~mm}$ photographic negative taken on 1987 December 20.13 UT. The two novae are clearly not spatially coincident. Updated astrometry has been performed, and revised coordinates measured, for both M31N 1924-08a and 1987-12a, and are given in Table 2 .

\subsubsection{M31N 1925-07c}

M31N 2011-12b (PNV J00435583+4121265), which was discovered by K. Nishiyama and F. Kabashima on 2011 December 27.448 UT at $m=17.4$ (unfiltered), was found to lie $\sim 7.5^{\prime \prime}$

from the position of Nova \#49 in Hubble's survey (Hubble 1929). Despite the relatively large reported separation, our expanded initial screen for early photographic surveys flagged this pair of novae for closer inspection. Figure 23 shows a comparison of the positions of M31N 1925-07c and 2011-12b, revealing that the novae to be distinct objects. Revised coordinates for M31N 1925-07c are given in Table 2 . 


\subsubsection{M31N 1925-09a}

M31N 1976-12a was discovered at $B=18.3$ by Rosino et al. (1989) as the 115th nova in his survey. Our coarse screen reveals that Rosino \#115 lies within 9.7" of a very bright

nova $\left(m_{p g}=15.3\right)$, which is nova \#54 in the survey of Hubble (1929). Given the disparity in peak brightness, and the relatively large apparent separation, we considered it unlikely that the 1976 eruption was a recurrence of Hubble \#54. Nevertheless, given that the novae are relatively far from the nucleus of M31 $\left(a \simeq 20^{\prime}\right)$, the probability for a chance coincidence is relatively low $\left(P_{C} \simeq 0.155\right)$, and we decided to take a closer look at the positions of these two novae. The positions are compared in Figure 24, confirming that the novae are in fact distinct objects. Revised coordinates for M31N 1925-07c are given in Table 2.

\subsection{8. $M 31 N 1927-08 a$}

M31N 1927-08a was discovered as nova \#76 in the survey of Hubble (1929), reaching a magnitude of $m_{p g}=17.1$ on 1927 August 23 UT. A possible, but unlikely recurrence with a reported position $\sim 8^{\prime \prime}$ from 1927-08a, the $R=17.4$ nova M31N 2009-11e (Pietsch et al. 2009b), was flagged by our screen. Figure 25 shows finding charts for the two novae, and clearly establishes that they are in fact distinct objects.

\subsubsection{M31N 1930-06b}

M31N 1930-06b was reported by Mayall (1931). The object was detected on Mount Wilson plates taken on 1929 Nov 30 and 1930 June 19. Both times the apparent nova was seen at $m \sim 18$. A possible recurrence, M31N 2008-08e, was discovered by D. Balam on 2008 August 31. Unpublished observations by one of us (KH) at the Ondrejov Observatory, establish that the object is visible on multiple images taken over a two-year period from March 2007 to August 2008. Given its photometric behavior, the object appears to be a long period variable star and not a nova in M31.

\subsubsection{M31N 1930-06c}

M31N 1996-08a (Shafter \& Irby 2001) was observed to erupt within $s \sim 7.2^{\prime \prime}$ of the reported position of M31N 1930-06c (nova \#92 in Mayall 1931) and made it through our coarse screen for RN candidates. A review of plate $\mathrm{H} 1155 \mathrm{H}$ in the Carnegie archives taken 
on 1930 June 28 fails to reveal a source at the reported position of M31N 1930-06c. Instead, an object labeled "nova 92" is found far from the reported position of M31N 1930-06c on the opposite (South) side of the nucleus of M31. A comparison of the position of Hubble's nova \#92 with that of M31N 1996-08a is shown in Figure 26. We conclude that an error was made in the conversion of Hubble's original $\mathrm{X}$ and $\mathrm{Y}$ position of the nova to the equatorial coordinates for M31N 1930-06c given in the online M31 nova catalog. Revised coordinates for both novae are given in Table 2 .

\subsubsection{M31N 1953-11a}

M31N 1953-11a was discovered by Arp (1956) as nova \#19 in his survey. Three subsequent novae were seen to erupt nearby and passed our coarse screen: M31N 1960-12a, 1962-11b, and 2013-05b. Of these, M31N 1962-11b (Rosino 1964) was the closest to 1953$11 \mathrm{a}$, with a reported position lying just $\sim 2.9^{\prime \prime}$ away. These novae are all relatively close to the nucleus of M31 $\left(a \sim 4.4^{\prime}\right)$, making the probability of a chance positional coincidence between M31N 1953-11a and 1962-11b relatively high $\left(P_{C} \simeq 0.622\right)$. As with M31N 1953-09b, we were able to locate the original Arp plate (S1137A) in the Carnegie archives. A reproduction of the nova field, along with that of M31N 1962-11b, is shown in Figure 27. The novae are clearly not spatially coincident. Furthermore, as expected, a comparison of Figure 27 with Figure 8 shows that both M31N 1953-11a and 1962-11a are also not coincident with either M31N 1960-12a or 2013-05b. However, as discussed earlier and shown in Figure 8 , the latter two novae are in fact spatially coincident to within observational uncertainties, strongly suggesting that M31N 1960-12a is a RN. Revised coordinates for M31N 1953-11a have been included, along with those of 1960-12a and 1961-11b, in Table 2.

\subsubsection{2. $M 31 N 1954-06 c$}

M31N 2010-01b was discovered by Nishiyama et al. (2010) on 2010 January 17.438 UT at $m=18.1$. The position of the nova lies $\sim 8.8^{\prime \prime}$ (just inside our coarse screen) from the position of M31N 1954-06c, which is Nova \#28 in Arp's nova survey (Arp 1956). The light curve of M31N 1954-06c is very slow, with Arp (1956) finding a "duration" (the number of days where the nova is brighter than $m=20$ ) of 115 days. In contrast, light curve information on 2010-01b is much more sparse; however, photometry by Pietsch \& Henze (2010) shows that the nova remained near $m=17.7$ between 2010 January 25 and February 02. As with

the previous two novae from Arp's survey, we located the discovery plate (S1335A) in the Carnegie archives, and re-measured the coordinates of the nova (see Table 2). The field of 
M31N 1954-06c is compared with that of 2010-01b in Figure 28, clearly showing that the two novae are not spatially coincident.

\subsubsection{M31N 1955-09b}

M31N 2012-03b erupted 2.5" from the reported position of M31N 1955-09b, nova \#1 in the extensive survey of Rosino (1964). The pair of novae are located at an isophotal radius of $a \sim 10.5^{\prime}$ from the center of M31, resulting in a relatively low probability of chance

coincidence, $P_{C} \simeq 0.032$. Finding charts for the two novae are shown in Figure 29. The two novae are clearly not spatially coincident, and thus M31N 2012-03b is not a recurrence of 1955-09b.

\subsubsection{M31N 1964-12b}

M31N 1964-12b (Rosino 1973) erupted very close to the nucleus of M31 $\left(a \simeq 0.71^{\prime}\right)$. A possible recurrence, M31N 1998-07b (Rector et al. 1999), lying 3.74" from that of 1964-12b was flagged by our screen. After re-analyzing the original data from the RBSE project, we have determined that M31N 1998-07b was a spurious detection, and the nova does not exist.

\subsubsection{5. $M 31 N 196 \%-11 a$}

M31N 1967-11a was discovered on archival plates by Henze et al. (2008a). A possible recurrence, M31N 2006-02a, was later discovered by K. Hornoch (Pietsch et al. 2007a). Charts for the two novae are shown in Figure 30. Although the positions of the novae appear close, a careful inspection reveals that the positions differ, with M31N 2006-02a located $\sim 4^{\prime \prime}$ WSW of the position of 1967-11a, in agreement with their reported positions.

\subsubsection{M31N 1967-12b}

M31N 1967-12b is another nova discovered by Rosino (1973). Its reported position is close to that of M31N 1999-06b discovered later as part of the RBSE program (Rector et al. 1999). Charts for the two novae are shown in Figure 31. The novae are clearly not spatially coincident, with M31N 1999-06b lying 6" ESE of 1967-12b. Refined coordinates for M31N 1967-12b are given in Table 2 . 


\subsubsection{M31N 1969-08a}

M31N 2007-12b erupted very close to the reported position of M31N 1969-08a. Nevertheless, as discussed by Bode et al. (2009), the objects have been shown to be distinct novae. Interestingly however, Bode et al. (2009) were able to show that M31N 2007-12b has a red giant companion star. This discovery, combined with its He/N spectroscopic type, makes it likely that M31N 2007-12b has a short recurrence time. Thus, it is possible that it will be revealed to be a $\mathrm{RN}$ in the not too distant future. We also note that Pietsch et al. (2011) found a 1110 s coherent periodicity in the X-ray light curve of M31N 2007-12b, which they interpreted as the rotation period of a magnetized white dwarf in the system, and suggested that the nova erupted from a intermediate polar progenitor.

\subsubsection{M31N 1975-09a}

M31N 1975-09a was discovered by Henze et al. (2008a) from an analysis of the Tautenburg Schmidt plates. Henze et al. (2008a) explored the possibility that M31N 1999-01a was a recurrence of 1975-09a and determined that the novae were not spatially coincident. We confirm their conclusion as can be seen in Figure 32.

\subsubsection{M31N 197r-12a}

M31N 1977-12a is nova \#119 from Rosino et al. (1989). A possible recurrence is M31N 1998-08a, which was reported to erupt only $2.1^{\prime \prime}$ from the nominal position of 1977-12a. Given that the novae were observed at a relatively large galactocentric radius $\left(a=10.8^{\prime}\right)$, the probability of a chance positional coincidence is small $\left(P_{C} \simeq 0.054\right)$. Despite the likelihood that the novae are related, comparison of the finding charts (Fig. 33) shows that the novae are in fact distinct. Revised astrometry of M31N 1977-12a (see Table 2) reveals that the separation between the eruptions is slightly larger, with 1977-12a lying 2.9" ESE of 199808 .

\subsubsection{0. $M 31 N$ 1992-12b}

M31N 1992-12b was discovered by Shafter \& Irby (2001) at an isophotal radius of $a \simeq 12.91^{\prime}$ from the center of M31. A possible recurrence, M31N 2001-10f was seen to erupt $\sim 5.31^{\prime \prime}$ from the position of 1992-12b. Given this large separation, it is unlikely that 
M31N 2001-10f is related to 1992-12b, despite the modest probability of a chance positional coincidence $\left(P_{C} \simeq 0.256\right)$. Revised astrometry of M31N 1992-12b given in Table 2 shows that the position is within $\sim 0.8^{\prime \prime}$ of that given in Shafter \& Irby (2001). The positions of M31N 1992-12b and 2001-10f are shown in Figure 34, making it clear that the two novae are distinct objects.

\subsubsection{M31N 1993-09b}

M31N 1993-09b was another nova discovered in the H $\alpha$ survey of Shafter \& Irby (2001). Approximately 3 years later, a possible recurrence, M31N 1996-08g (Sharov \& Alksnis 1997; Henze et al. 2008a), was observed $\sim 5.47^{\prime \prime}$ away from the position of 1993-09b. The probability of a chance positional coincidence is relatively high with $P_{C} \simeq 0.843$. Figure 35 clearly establishes that the two novae are distinct objects. Revised coordinates for M31N 1993-09b are given in Table 2 .

\subsubsection{2. $M 31 N$ 2001-08d}

M31N 2001-08d was discovered in the bulge of M31 $\left(a \simeq 4.34^{\prime}\right)$ by Fiaschi et al. (2001) on an $\mathrm{H} \alpha$ image of M31 taken on 2001 September 02.93 UT. A possible recurrence, M31N 2008-07a (Henze et al. |2008b), was observed within $\sim 3.5^{\prime \prime}$ of the reported position of 2001$08 \mathrm{~d}$ with an estimated probability of change positional coincidence given by $P_{C} \simeq 0.774$. A comparison of finding charts for the two novae (Fig. 36) shows that the two novae are not positionally coincident.

\subsubsection{3. $M 31 N 2004-11 b$}

M31N 2004-11b (Lee at al. 2012) and 2010-07b (Hornoch et al. 2010c) represent two nova outbursts in the bulge of M31 $\left(a \simeq 5^{\prime}\right)$ with reported positions within $\sim 5.9^{\prime \prime}$ of one another. Not surprisingly, given that the coordinates are unlikely to be in error by more than an arcsec, Figure 37 confirms that the two novae are not spatially coincident. 


\subsubsection{4. $M 31 N$ 2005-05b}

M31N 2005-05b (Lee at al. 2012) and M31N 2009-08d (Henze et al. 2009a) erupted within $\sim 5^{\prime \prime}$ of one another, and very close to the nucleus $\left(a \sim 0.8^{\prime}\right)$, resulting in a relatively high probability of a chance positional coincidence $\left(P_{C} \simeq 0.998\right)$. The finding charts from the RBSE data shown in Figure 38 reveal, as expected, that the objects are distinct systems.

\subsubsection{M31N 2006-11b}

M31N 2006-11b and M31N 2006-12d (Lee at al. 2012) are two cataloged novae observed near the nucleus of M31 with reported positions within $0.34^{\prime \prime}$ of one another. The probability of a chance coincidence is small $\left(P_{C} \simeq 0.029\right)$. The objects appear to be spatially coincident, but it seems clear that M31N 2006-12d, observed just 38 days later, is a re-brightening of 2006-11b, and not a distinct eruption.

\subsubsection{6. $M 31 N 2006-12 c$}

M31N 2006-12c and M31N 2007-07e (Lee at al. 2012) erupted close to the nucleus $\left(a \sim 2^{\prime}\right)$, and within $\sim 4.4^{\prime \prime}$ of one another resulting in a high probability of a chance positional coincidence. Once again, finding charts for the objects (see Fig. 39) reveal that they are distinct systems.

\subsubsection{M31N 2010-01a}

M31N 2010-01a (Burwitz et al. 2010) was discovered close to the nucleus of M31 $\left(a \simeq 2.7^{\prime}\right)$, and observed to have a possible recurrence less than a year later, M31N 2010-12c (Hornoch et al. 2010a; Ruan \& Gao 2010b), which was reported just 0.82" away. Despite a probability of a chance positional coincidence of just $P_{C} \simeq 0.141$, a careful inspection of the images for each nova shows that they are in fact distinct objects (see Figure 40). This conclusion is supported by the work of Hornoch et al. (2010a), who performed careful astrometry of both novae and also came to the conclusion that they are in fact distinct systems. Revised coordinates based on astrometry performed on the charts shown in Figure 40 are given in Table 2. We conclude that M31N 2010-01a is not a RN. 


\section{The Recurrent Nova Sample in M31}

Based on the analysis of the RN candidates from Table 1 as described in the preceding section, we have determined that there are a total of at least $12 \mathrm{RNe}$ that have been observed in M31 over approximately the past century. Four additional systems, M31N 1953-09b, 196111a, 1966-08a, and 1990-10a are considered possible RNe. These 16 RN systems, representing

39 independent nova eruptions, are summarized in Table 3 , where we have recalculated the observed separation, $s$, and updated the probabilities for chance positional coincidence, $P_{C}$, to reflect the revised coordinates given in Table 2. We note that since not all of the RNe identified above have been spectroscopically confirmed, we cannot completely eliminate the possibility that one or more LPVs could be lurking in our RN sample.

\subsection{Spatial Distribution}

Figure 41 shows the spatial distribution of the $12 \mathrm{RNe}$ and the 4 possible RNe compared with all novae in our sample. There is no obvious difference in the overall spatial position of the RNe compared with that of novae in general. To explore the distributions more quantitatively, we have plotted the cumulative distributions of the RNe and the remaining nova sample as a function of isophotal radius in Figure 42. For comparison, we have included the cumulative $B$-band light for M31 as well as an estimate of the cumulative bulge light. The cumulative $B$-band light was computed from the surface photometry of de Vaucouleurs (1958), with the cumulative bulge light computed from a standard $r^{1 / 4}$ law

$$
\mu(r)=\mu_{e}+8.33\left[\left(r / r_{e}\right)^{1 / 4}-1\right]
$$

parameterized by $\mu_{e}=22.9 \mathrm{mag} \operatorname{arcsec}^{-2}$ and $r_{e}=18^{\prime}$ for $B$-band light as found by Ciardullo et al. (1987).

It is clear from Figure 42 that there is no discernible difference between the cumulative distributions for $\mathrm{RNe}$ and novae in general $(\mathrm{KS}=0.95)$, however both distributions differ appreciably from the integrated bulge and (especially) total light. The poor agreement between the nova distributions and the light distributions is not unexpected, and can be explained by the fact that the combined nova sample is far from being spatially complete. The Andromeda galaxy covers a relatively large angular area with the major axis extending more than 3 degrees on the sky. Most M31 nova surveys over the years have concentrated on the inner (bulge dominated) regions of the galaxy, with extended coverage being far more sporadic. As a result, the cumulative nova samples are observed to fall off faster than the 
cumulative background light, with the bulge light providing a better fit than the total (bulge + disk) light. In cases where the nova distribution from a given spatially-defined survey is compared with the cumulative light included in that particular survey, the nova distributions have been shown to agree quite well with the integrated bulge (but not the total) light (e.g., see Ciardullo et al. 1987; Shafter \& Irby 2001; Darnley et al. 2006).

\subsection{Optical and X-Ray Outburst Properties}

The optical and X-ray outburst properties of the full sample of RNe are summarized in Table 4. The optical properties include the outburst discovery magnitude, $m_{\text {dis }}$, and when known, the time for the nova to fade by two magnitudes $\left(t_{2}\right)$ from maximum light. X-ray properties have been measured for five RNe, including the turn-on and turn-off times $\left(t_{\mathrm{on}}\right.$, $\left.t_{\text {off }}\right)$ for the SSS phase of the eruption, and the best-fit blackbody temperature, $T_{\mathrm{bb}}$, for three of the systems. When known, we also have included the spectroscopic class of the nova. Unfortunately, very little photometric or spectroscopic data are available for most of the initial RN outbursts, mostly because the first recorded outbursts occurred prior to the time that routine imaging and spectroscopic observations became feasible. In no cases do we have spectroscopic classes available for the initial outburst, and in only two cases (M31N 1963-09c and 1997-10f) do we have estimates of the rate of decline from maximum light. The $t_{2}$ times for M31N 1963-09c and 1997-10f are given by $t_{2} \gtrsim 17 \mathrm{~d}$ and $t_{2} \sim 10 \mathrm{~d}$,

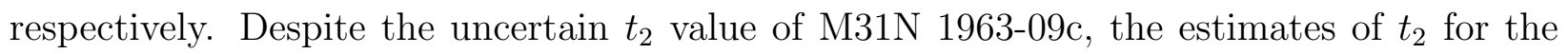
three recurrences are consistent with one another, and establish that the nova declined rapidly. In addition, Shafter et al. (2010) established that 2010-10e belonged to the He/N spectroscopic class. Thus, both the photometric and spectroscopic properties are consistent with the identification of M31N 1963-09c as a RN. In the case of M31N 1997-10f and its likely recurrence 2008-08b, we have no estimate of the $t_{2}$ value of the latter eruption, however an optical spectrum by di Mille et al. (2010) shows that the object is a likely member of the $\mathrm{He} / \mathrm{N}$ class.

The remarkable RN system M31N 2008-12a displayed 4 observed recurrences through the end of 2013: 2009-12b, 2011-10e, 2012-10a, and 2013-11f (e.g., see Darnley et al. 2014a; Henze et al. 2014b; Tang et al. 2014). Available photometry for two of the outbursts (201110e and 2013-11f) is sufficient to establish that the nova faded extremely rapidly $\left(t_{2} \simeq 2 \mathrm{~d}\right)$, while spectroscopic observations of the last two eruptions establish that the nova is a member of the He/N spectroscopic class. Such systems comprise approximately $15 \%$ of novae observed in M31, with the remaining 85\% mostly belonging to the Fe II class (Shafter et al. 2011b). The former novae are characterized by relatively high ejection velocities (FWHM of $\mathrm{H} \alpha$ 
typically $\gtrsim 2500 \mathrm{~km} \mathrm{~s}^{-1}$ ) and rapid photometric development as expected from a nova arising from a massive white dwarf. As explained in Henze et al. (2014b), the relatively modest peak optical absolute magnitude of $M_{\max } \sim-6$ is consistent with the extremely short recurrence period of this RN.

Of the remaining RN systems in M31, a total of 4 more have spectroscopic classifications. Two of these 4 have been classified as Fe II systems (M31N 2007-08d and 2009-11b), with the other two belonging to the He/N (2012-01b, Shafter et al. 2012c) or broad-lined Fe II (201209a, Shafter et al. 2012e) classes. Thus, among the $7 \mathrm{RN}$ systems with known spectroscopic types, 5 are classified as either $\mathrm{He} / \mathrm{N}$ or Fe IIb.

Of the 12 RNe and the 4 possible RNe systems, five were detected in X-rays as SSS: M31N 1919-09a (as M31N 1998-06a), M31N 1953-09b (as M31N 2004-08a), M31N 1963-09c (as M31N 2010-10e), M31N 1984-07a (as M31N 2004-11f), and M31N 2008-12a (as M31N 2013-11f). Four of these sources displayed very short SSS phases $(<100$ days) with high effective X-ray temperatures (only measured for three of them). Short SSS time scales and high effective temperatures have been found to be correlated in M31 novae (Henze et al. 2014a) and are believed to indicate a high white dwarf mass (Hachisu \& Kato 2006). Therefore, their X-ray properties are consistent with these objects being RNe, which require a high white dwarf mass and a high accretion rate to produce frequent eruptions. The notable exception from this picture is M31N 1919-09a, which was visible as a SSS between 1000 and 2000 days after its 1998 outburst. Although this property points towards a significantly lower white dwarf mass than for the other RNe, the available X-ray data is insufficient to challenge seriously the classification of M31N 1919-09a as a RN.

\section{The Recurrent Nova Fraction in M31}

The 12 highly probable RN systems identified above have produced a total of $n_{\text {out }}(\mathrm{RNe})=$ 32 nova outbursts out of the total of 964 outbursts observed in M31 up to the end of calendar year 2013. From these data we find that the observed ratio of RN to CN outbursts in M31 is given by:

$$
n_{\text {out }}(\mathrm{RNe}) / n_{\text {out }}(\mathrm{CNe})=n_{\text {out }}(\mathrm{RNe}) /\left[964-n_{\text {out }}(\mathrm{RNe})\right]=0.0343 \text {. }
$$

If we consider all plausible RNe, including the 4 systems ( 8 outbursts) classified as uncertain, $n_{\text {out }}(\mathrm{RNe})=40$, and we have: 


$$
n_{\text {out }}(\mathrm{RNe}) / n_{\text {out }}(\mathrm{CNe})=0.0433 \text {. }
$$

Given the uncertainties, in the analysis to follow we adopt a mean value of

$$
n_{\text {out }}(\mathrm{RNe}) / n_{\text {out }}(\mathrm{CNe}) \simeq 0.04
$$

(one in 25) as a reasonable estimate of the ratio of recurrent to classical nova outbursts observed in M31. It is clear, however, that this ratio must be a lower limit to the true ratio of recurrent-to-classical nova outbursts in the galaxy given that the sporadic temporal and uneven spatial coverage of M31 surveys over the past century has impacted the discovery efficiency for RNe, where at least two outbursts must be observed, considerably more than it has the discovery of systems classified as CNe.

\subsection{Estimating the Discovery Efficiencies for $\mathrm{CNe}$ and $\mathrm{RNe}$}

In order to accurately estimate how the discovery efficiencies of $\mathrm{CNe}$ and $\mathrm{RNe}$ differ in M31, it would be necessary to know the exact temporal and spatial coverage as well as the limiting magnitudes of the many M31 surveys that have taken place over the past century. In addition, the typical recurrence times and light curve properties of both $\mathrm{RNe}$ and CNe would need to be known. Obviously, the precise values for all of these parameters are not available. However, we can estimate the expected ratio of RN to CN outbursts from numerical simulations that are based on plausible values for these unknowns. Before describing the simulations, we first turn to a discussion of the important biases that will affect the analysis.

\subsubsection{Temporal and Spatial Biases of M31 Nova Surveys}

The history of nova discoveries in M31 is shown in Figure 43. A few notable features are apparent. The first clump of discoveries centered in the 1920s is due largely to Hubble's

pioneering survey (Hubble 1929). Nova discoveries tailed off precipitously in the 1930s and essentially dropped off completely during World War II. Nova discoveries picked up significantly in the 1950s and 1960s as a result of the Arp (Arp 1956) and Rosino (Rosino 1964 , 1973 photographic surveys. Then, after a short lull in the 1970s, nova discoveries began a steady increase due primarily to the CCD surveys of Ciardullo et al. (1987), Shafter \& Irby (2001), Darnley et al. (2006), and others. In recent years the number of novae discovered in 
M31 has exploded thanks to the contributions from automated surveys such as the Palomar Transient Factory (Law at al. 2009), and amateur astronomers throughout the world.

In addition to the overall number of novae discovered over the past century, Figure 43 also shows the distribution of RN outbursts observed over time. As expected, the number of outbursts of RN systems has increased roughly in proportion to the number of nova discoveries. However, despite the dramatic increase in the overall number of nova discoveries in recent years, the first recorded eruptions of our $12 \mathrm{RNe}$ and our 4 possible RNe are spread relatively evenly over time. This result is not surprising. If surveys had been uniform over time, with the discovery of novae increasing at a steady rate, we would have expected the percentage of novae identified as recurrent to be higher for systems discovered early on, where more would be available for the discovery of subsequent outbursts. The fact that the density of observations has increased over time has blunted this effect and resulted in what we see, namely that the identification of new RN systems is approximately evenly distributed over the past century.

In addition to the sporadic temporal coverage, the uneven spatial coverage of the various surveys can be expected to impact the relative discovery efficiencies of RNe and CNe. In particular, the discovery efficiency of RNe should be biased towards the bulge of the galaxy where the temporal coverage is more extensive. From a theoretical standpoint, it is unclear whether the recurrence times of novae (and hence the relative populations of $\mathrm{RNe}$ and $\mathrm{CNe}$ ) should vary with spatial position in M31. Population synthesis models predict that younger stellar populations should produce nova progenitors that have on average more massive white dwarfs than do older stellar populations (e.g., de Kool 1992 Politano 1996). Since the recurrence times of novae are strongly dependent on the mass of the white dwarf (e.g., Yaron et al. 2005: Wolf et al. 2013), younger stellar populations should not only produce a higher observed nova rate (Yungelson et al. 1997), but also a higher fraction of systems classified as RNe. Despite these predictions, observations have consistently shown that novae are more prominent in older bulge populations (e.g., Ciardullo et al. 1987; Shafter \& Irby 2001; Darnley et al. 2006). The fact that the observed spatial distribution of RNe does not differ significantly from that of novae generally (see Figure 42) suggests that an unbiased survey (with more complete disk coverage) may find that RNe are more spatially extended than novae in general. If so, such a finding would be consistent with the predictions of the population synthesis models. 


\subsubsection{Limiting Magnitudes of M31 Nova Surveys}

Aside from the temporal and spatial uncertainties, we must also consider how variations in the limiting magnitudes of the observations will impact the discovery of novae in M31. Limiting magnitudes for most of the M31 surveys vary significantly, both between surveys and within the surveys themselves. Thus, it is not possible to determine any single limiting magnitude representative of the full M31 nova sample. Nevertheless, we can get an idea of the limiting magnitude of the "average" survey by referring to Figure 44, which shows the distribution of discovery magnitudes for the 964 M31 nova candidates in the Pietsch et al. (2007a) online database. Here we assume that the average survey reaches a limiting magnitude down to the point where the discovery magnitude distribution turns over and begins to drop precipitously. Based on the distribution of discovery magnitudes, it appears that a limiting magnitude of $m_{\lim } \sim 18$ is representative of the typical M31 nova survey. Given the considerable uncertainty in $m_{\mathrm{lim}}$, in the simulations described below we have also considered how our results are affected by adopting limiting magnitudes of $m_{\lim }=17$ and 19.

\subsubsection{Monte Carlo Simulations}

We have performed a series of Monte Carlo simulations to predict the discovery efficiencies of both $\mathrm{CN}$ and RN outbursts in M31 under a variety of scenarios. In the simulations, we produce a total of 6500 synthetic novae erupting at random times over a 100 year time span ${ }^{7}$. Model nova light curves are based on the the maximum magnitudes and rates of decline from the "high quality" M31 sample of Capaccioli et al. (1989) and from the Galactic RN sample from Schaefer (2010) for CNe and RNe, respectively. Table 5 gives the values of $m_{B}(\max )$ and $\nu_{B}\left(\operatorname{mag} \mathrm{d}^{-1}\right)$ assuming a distance and foreground reddening for M31 of $(m-M)_{o}=24.38$ and $A_{B}=0.25$ (Freedman et al. 2001; Schlegel et al. 1998). For CNe, an eruption reaches a maximum brightness and then fades at a rate that is chosen at random from the CN light curve parameters given in Table 5. A model nova is considered "detected" if it remains brighter than the adopted limiting magnitude $\left(m_{\lim }=17,18\right.$, or 19$)$ on the nearest survey date immediately following the date of the simulated eruption. The fraction of the 6500 novae that are detected is reported as the discovery efficiency for CNe.

As a lower limit on the temporal coverage of M31 we have simply taken the discovery

\footnotetext{
7 We have chosen 6500 eruptions based on an estimated annual nova rate of $65 \mathrm{yr}^{-1}$ (Darnley et al. 2006), although the predicted ratio of RNe to CNe outbursts is not sensitive to the assumed nova rate.
} 
dates of the nova candidates that have been discovered in M31 over the past century. Then, to explore the sensitivity of our simulations to the temporal coverage, we have also considered the possibility that the actual survey coverage extended one or two days on either side of each of the dates were a nova was reported to be discovered. We refer to the temporal coverage estimates either as "Clump 1" (discovery dates only), "Clump 3" (discovery dates plus the day immediately preceding and following each discovery date), or "Clump 5" (discovery date plus the 2 days preceding and the 2 days following all discovery days). It is also possible that there were more extended intervals of coverage where no novae were discovered, but we have no way of accurately accounting for this possibility.

In the case of RNe, our simulations must produce multiple outbursts, and the RN discovery efficiencies will clearly depend on the assumed recurrence time, or the distribution of recurrence times, adopted in the simulations. To explore this dependence, we have initially considered several trial recurrence times ranging from as short as 1 year (the shortest observed RN recurrence time) to a maximum of 90 years (longer recurrence times have a negligible chance of being detected in our simulations that span just 100 years). As for CNe, the light curve parameters for RNe have also been chosen randomly, this time from the RN sample given given in Schaefer (2010) and summarized here in Table 5.

To qualify as a RN outburst in our simulations, a particular system must be detected at least twice over the 100 year time span of the simulation. The RN discovery efficiency is computed as the ratio of the total number of multiple outbursts detected to the total number of outbursts generated in the RN simulation (i.e., 6500 simulated nova outbursts plus all recurrences in the 100 year interval). The entire simulation for both $\mathrm{CNe}$ and $\mathrm{RNe}$ has been repeated a total of 100,000 times, with the CN outburst fraction, the RN outburst fraction recorded as the respective discovery efficiencies.

The results of our Monte Carlo simulations of the RN outburst discovery efficiencies and their ratio to that of $\mathrm{CNe}$ are shown in Figure 45. Not surprisingly, the discovery efficiencies are strongly dependent on the assumed limiting magnitude of the surveys, with the deeper surveys resulting in higher $\mathrm{RN}$ discovery efficiencies. The assumed density of the temporal coverage is also important, with the discovery efficiencies increasing with increasing temporal coverage, as expected. It is interesting to note that at the shortest recurrence times, the discovery efficiencies for RNe relative to CNe for $m_{\lim }=18$ slightly exceeds that for $m_{\lim }=19$ for the "Clump 3" and "Clump 5" temporal distributions. This behavior results from the fact that the CN light curves have generally slower rates of decline when compared with the RN light curve sample. A fainter limiting magnitude thus increases the CN discovery efficiency more than it increases the RN discovery efficiency. This effect is most pronounced for the shorter recurrence times and denser temporal coverage where the $\mathrm{RN}$ discovery efficiency is 
already quite high.

Regardless of the limiting survey magnitude or the temporal coverage, these simulations confirm that the observed RN recurrence time distribution for known RNe should strongly favor the discovery of RNe having shorter recurrence times. To verify this prediction, in Figure 46 we have plotted the observed outburst distribution for Galactic and M31 RNe, and compared it with the $m_{\lim }=18$, "Clump 3" discovery efficiencies from Figure 45. Since we simulated the same number of RNe across all recurrence times, the qualitative agreement shown in Figure 46 is consistent with the hypothesis that the observed bias toward the discovery of short recurrence time RNe can be explained solely by selection bias from an intrinsically flat distribution of RN recurrence times.

Under the assumption that the intrinsic $\mathrm{RN}$ recurrence time distribution is flat over the range of $1-100$ years, we have conducted two composite recurrence time RN simulations. In the first composite simulation we choose recurrence times at random from the full range of $t_{\text {rec }}=1-100$ years. Then, we consider a restricted range with recurrence times ranging from $t_{\text {rec }}=5-100$ years that would be more appropriate for a population of RNe where ultra-short recurrence time RNe such as M31N 2008-12a are extremely rare.

The results of the Monte Carlo simulation are reported in Table 6 and shown in Figures 47 and 48 for the various input parameters. Columns 3 and 4 of Table 6 give the fractions of $\mathrm{CN}$ and RN outbursts that are detected in the simulation, with column 5 giving the principal result of our simulation, the ratio of RN to CN outbursts detected (see Figs. 47, 48). Column 6 gives our estimate of the true ratio of RN to CN outbursts, $N_{\text {out }}(\mathrm{RNe}) / N_{\text {out }}(\mathrm{CNe})$, after the observed outburst ratio given in Equation (5) is corrected for the relative outburst discovery efficiencies. Columns 7 and 8 then give our estimates of the expected numbers of RN and CN outbursts produced in M31 each year assuming a combined annual nova rate of $65 \mathrm{yr}^{-1}$ (Darnley et al. 2006).

As a check on our simulations, we note that the CN discovery efficiency predicted by our Monte Carlo simulations is in qualitative agreement with observations. Given an annual nova rate of $65 \mathrm{yr}^{-1}$, we estimate that approximately 6500 novae have erupted in M31 over the past century. Our standard model $\left(m_{\lim }=18\right.$, "Clump 3") produces a CN discovery fraction of 0.15 and predicts that approximately 975 novae will have been discovered over the past century, in very good agreement with the number of novae discovered in M31. It is also worth noting that our values for the true ratio of RN to CN outbursts, $N_{\text {out }}(\mathrm{RNe}) / N_{\text {out }}(\mathrm{CNe})$, for our $m_{\lim }=18,19$ models are consistent with the ratio estimated by Della Valle \& Livio (1996) in their study of the RN populations in the Galaxy, the LMC, and M31.

Under the assumption that the recurrence time distribution is flat in the range of $1-100$ 
years, we predict that $\mathrm{RN}$ outbursts make up $\sim 10 \%$ of nova eruptions generally. For an annual nova rate of $65 \mathrm{yr}^{-1}$, as many as $5-10$ outbursts per year are expected to arise from RN progenitors. If ultra-short recurrence time $\left(t_{\text {rec }}<5 \mathrm{yr}\right) \mathrm{RNe}$ are relatively rare, our $5<t_{\text {rec }} /$ yr $<100$ models suggest that as many as one out of three $(\sim 20)$ nova eruptions observed annually in M31 could arise from RN systems. This result is consistent with that found recently by Pagnotta \& Schaefer (2014) for that fraction of Galactic novae that are recurrent, and Williams et al. $(2014,2015)$ for the fraction of M31 novae with evolved secondary stars.

\section{RNe as a Channel for the Production of Type Ia Supernovae}

SNe Ia are thought to arise from the deflagration of an accreting white dwarf in a binary system (Maoz \& Mannucci 2012). There are two primary models for the progenitor systems (1) a single degenerate channel, where the white dwarf accretes from a companion star in a semi-detached binary, and (2) a double-degenerate channel, where a pair of orbiting white dwarfs merge as a result of the loss of orbital energy and angular momentum from the emission of gravitational waves. RNe are sometimes proposed as potential contributors to the single degenerate channel. However, the consensus has generally been that RNe do not exist in sufficient numbers to explain the observed rate of SNe Ia production in a galaxy like M31.

Based on our estimates for the RN rate in M31, we can explore whether these systems might make a significant contribution to the production of SNe Ia in a galaxy like M31. If we assume RNe have a mean mass accretion rate, $\dot{M}=10^{-7} \mathrm{M}_{\odot} \mathrm{yr}^{-1}$, which is typical for Galactic RN systems (Schaefer 2010), and that each RN system must accrete a total of 0.3 $\mathrm{M}_{\odot}$ of material before reaching the Chandrashakhar limit, it would take approximately 3 million years on average for a given $\mathrm{RN}$ to become a SNe Ia. Assuming a RN rate of as many as 20 per year and a mean recurrence time of 30 years between outbursts, we estimate that there could be as many as 600 active RN systems in M31. This number likely represents an upper limit to the number of active RN systems in M31 given that most models predict fewer than 20 RN outbursts per year and the mean recurrence time is likely shorter than 30 years. Thus, we arrive at an estimated upper limit to the RN "death rate" of $\sim 2 \times 10^{-4} \mathrm{yr}^{-1}$, which could produce perhaps as many as one SNe Ia every 5000 yr in this galaxy.

Mannucci (2005) has estimated that for typical S0a/b and Sbc/d spirals, we can expect SNe Ia birthrates of $\sim 0.04$ and 0.08 SNe Ia per century per $10^{10} L_{\odot, K}$, respectively. Adopting $L_{K} \simeq 16 \times 10^{10} L_{\odot, K}$ for M31 (Shafter et al. 2013), gives expected SNe Ia rates of $\sim 0.64$ and $\sim 1.3$ per century depending on Hubble type. In the case of M31, with an intermediate 
Hubble type of Sbc, we estimate a rate of $\sim 1.0 \pm 0.4$ SNe 1a per century. Thus, it appears that RNe might be able to supply only $\sim 2 \%$ of the SNe Ia in a galaxy like M31. Given the uncertainties in the assumptions, this estimate clearly constitutes only a very rough estimate of the contribution that RNe might make to the production of SNe Ia in M31. Nevertheless, based on our analysis of the RN population in M31, it appears very unlikely that RNe could play a major role as SNe Ia progenitors.

\section{Conclusions}

We have undertaken a program to identify the fraction of RNe in the nearby spiral galaxy, M31. We have identified RN candidates by cross-correlating the published coordinates for 964 nova candidates recorded in M31 from 1909 September through the end of 2013. Allowing for a positional uncertainties $s<6^{\prime \prime}$ ( $9^{\prime \prime}$ for early photographic plate surveys), we have identified a total of 51 potential RN systems responsible for 118 observed eruptions. In order to determine which of these potential RNe are in fact RNe, we have made an attempt to locate and analyze original plates, films, CCD images, and finding charts for each of the 118 eruptions. We were successful in locating a number of images that have allowed us to identify a total of $12 \mathrm{RN}$ systems, with another 4 likely RNe, representing a total of 40 eruptions. In addition, we have been able to rule out a total of 27 potential RN systems representing a total of 61 individual eruptions. The remaining 8 systems, representing 17 eruptions, are unlikely to be RNe, but we have not been able to definitively rule out their RN nature.

Taking the 12 RN systems representing 32 eruptions, and assuming that 2 of the 4 possible RNe representing 4 eruptions are in fact recurrent, we find that approximately 1 in 25 of the observed nova outbursts in M31 arise from RN progenitors. To correct for observational bias against the detection of RN systems, we conducted a simple Monte Carlo simulation to estimate the relative discovery probability for $\mathrm{CN}$ and $\mathrm{RN}$ outbursts. For plausible estimates of the M31 observational coverage, we estimate that as many as one in three nova outbursts observed in M31 could arise from a population of RN progenitors having recurrence times less than a century. Despite the rudimentary nature of our simulation, which is unable to accurately account for the inhomogeneous spatial and temporal coverage of the M31 data set, this number is consistent with the estimate for Galactic RNe found by Pagnotta \& Schaefer (2014), and with the relatively high fraction of M31 novae found to harbor evolved secondary stars (Williams et al. 2014, 2015). Finally, based on our rough estimates for the number of RNe active in M31 and their expected white dwarf masses and accretion rates, we conclude that it appears unlikely that such systems can provide a 
significant channel for the production of Type Ia supernovae.

We would like to thank an anonymous referee for valuable comments and suggestions that have led to an improved presentation. We also thank S. M. Lauber for her assistance in searching for RN candidates in the RBSE data and for proof reading the manuscript. A.W.S. is grateful to C.T. Daub for discussions regarding the probabilities of chance positional coincidences of novae, and to J. Rice for discussions during the early stages of related work on RN in M31. This work is based in part on observations made with the Isaac Newton Telescope operated on the island of La Palma by the Isaac Newton Group in the Spanish Observatorio del Roque de los Muchachos of the Instituto de Astrofísica de Canarias. M.H. acknowledges support from an ESA fellowship. T.A.R. was funded for research on this project through NSF grants DUE-0920293 and DUE-0618849. K.H. was supported by the project RVO:67985815. A.W.S. acknowledges financial support through NSF grant AST-1009566. 


\section{REFERENCES}

Alksnis, A., \& Zharova, A. V. 2000, IBVS, 4909, 1

Alksnis, A., Smirnova, \& Zharova, A. V. 2008, Ast. Lett., 34, 563

Ansari, R. et al. 2004, A\&A, 421, 509

Arp, H. C. 1956. AJ, 61, 15.

Baade, W. \& Arp, H. 1964 ApJ, 139, 1027

Barsukova, E., Fabrika, S., Hornoch, K., Fatkhullin, T., Sholukhova, O., Pietsch, W. 2011, ATel \#3725

Barsukova, E. A., Fabrika, S., Vinokurov, A., Hornoch, K., Henze, M., Shafter, A. W. 2013, ATel \#5145

Bode, M. F., Darnley, M. J., Shafter, A. W., Page, K. L., Smirnova, O., Anupama, G. C., Hilton, T. 2009, ApJ, 705, 1056

Börngen, F. 1968, AN, 291, 19

Bryan, J. 1987, IAU Circ. \#4516, 2

Bryan, J. 1990, IAU Circ. \#5121

Burwitz, V. 2010, ATel \#2383

Cao, Y. et al. 2012, ApJ, 752, 133

Capaccioli, M., della Valle, M., Rosino, L., D’Onofrio, M. 1989, AJ, 97, 1622

Ciardullo, R., Ford, H. C., Neill, J. D., Jacoby, G. H., \& Shafter, A. W. 1987, ApJ, 318, 520

Darnley, M. J. et al. 2004, MNRAS, 353, 571

Darnley, M. J. et al. 2006, MNRAS, 365, 1099

Darnley, M. J. et al. 2014a, A\&A, 563, 9

Darnley, M. J. et al. 2014b, ATel \#6527

Darnley, M. J., Ribeiro, V. A. R. M., Bode, M. F., Hounsell, R. A., Williams, R. P 2012, ApJ, 746, 61 
Della Valle, M. \& Livio, M. 1996, ApJ, 473, 240

de Kool, M. 1992, A\&A, 261, 188

de Vaucouleurs, G. 1958, ApJ, 128, 465

Di Mille, F., Ciroi, S., Orio, M., Rafanelli, P., Bianchini, A., Nelson, T., Andreuzzi, G. 2008, ATel \#1703

Di Mille, F., Ciroi, S., Navasardyan, H., Orio, M., Rafanelli, P., Bianchini, A. 2009, ATel \#2171

Di Mille, F.; Orio, M.; Ciroi, S.; Bianchini, A.; Rafanelli, P.; Nelson, T. 2010, AN, 331, 197

Fabrika, S., Barsukova, E. A., Valeev, A. F., Sholukhova, O., Henze, M., Shafter, A. W., \& Hornoch, K. 2013, ATel \#5543

Fiaschi, M., Di Mille, F., Cariolato, R. 2001, IAU Circ. 7709

Fiaschi, M., Di Mille, F., Tiveron, D. 2003, IAU Circ. 8238

Freedman, W. L. et al. 2001, ApJ, 553, 47

Hachisu, I. \& Kato, M. 2006, ApJS, 167, 59

Hatzidimitriou, D., Burwitz, V., Duscha, S., Kanbach, G., Pietsch, W., Reig, P., Papamastorakis, G. 2007a, ATel \#1131

Hatzidimitriou, D., Burwitz, V., Steinle, H., Pietsch, W., Slowikowska, A., Reig, P., Papamastorakis, G. 2007b, ATel \#1156

Henze, M., Meusinger, H., \& Pietsch, W. 2008a A\&A, 477, 67

Henze, M. et al. 2008b, ATel \#1609

Henze, M. et al. 2008c, ATel \#1790

Henze, M. et al. 2009a, ATel \#2165

Henze, M. et al. 2009b, ATel \#2286

Henze, M., Ness, J.-U., Bode, M. F., Darnley, M. J., Williams, S. C., \& Shafter, A. W. 2013, ATel \#5633

Henze, M. et al. 2014a, A\&A, 563, 2 
Henze, M. et al. 2014b, A\&A, 563, 8

Hornoch, K., Prieto, J., Khan, R., Hornochova, P. 2010a, CBET, \# 2610

Hornochova, P., Hornoch, K., Wolf, M., Kusnirak, P., \& Pejcha, O. 2010b, CBET, \# 2573

Hornoch, K. et al. 2010c, CBET, \#2411

Hornoch, K. \& Galad, A. 2012, ATel \#4653

Hornoch, K., \& Vrastil, J. 2012a, ATel \#4364

Hornoch, K., \& Vrastil, J. 2012b, ATel \#3976

Hornoch, K., \& Vrastil, J. 2013, ATel \#5265

Hornoch, K., Kucakova, H., Gorosabel, J., Kubanek, P 2013, ATel \#5092

Hubble, E. P. 1929, ApJ, 69, 103

Kasliwal, M. M. 2009, ATel \#2290

Kato, M., Saio, H., Hachisu, I., Nomoto, K. 2014, arXiv 1404.0582

Law, N. M. et al. 2009, PASP, 121, 1395

Lee, C.-H., Riffeser, A., Seitz, S., Bender, R., Fliri, J., Hopp, U., Ries, C., Bärnbantner, O., Gössl, C. 2012, A\&A, 537, 43

Li, W. D. 2001, IAU Circ. 7674

Mannucci, F. 2005, ASP Conference Series, 342, 140

Mayall, N. U. 1931, PASP, 43, 217

Maoz, D.; Mannucci, F. 2012, PASA, 29, 447

Massey, P., Olsen, K. A. G., Hodge, P. W., Strong, S. B., Jacoby, G. H., Schlingman, W., Smith, R. C. 2006, AJ, 131, 2478

Nishiyama, K., Kabashima, F., Yusa, T. 2010, CBET \#2135

Pagnotta, A. \& Schaefer, B. E. 2014, ApJ, 788, 164

Pietsch, W., Fliri, J., Freyberg, M. J., Greiner, J., Haberl, F., Riffeser, A., Sala, G. 2005, A\&A, 442, 879 
Pietsch, W. et al. 2007a, A\&A, 465, 375

Pietsch, W. et al. 2007b, ATel \#201

Pietsch, W., Henze, M., Burwitz, V., Updike, A., Milne, P., Williams, G., Hartmann, D. H. 2009a, ATel \#1935

Pietsch, W., Podigachoski, P., Haberl, F., Henze, M., Burwitz, V., Updike, A., Milne, P., Williams, G., Hartmann, D. H. 2009b, ATel \#2308

Pietsch, W. \& Henze, M. 2010, ATel \#2435

Pietsch, W.; Henze, M.; Haberl, F.; Burwitz, V. 2010, ATel \#3038

Pietsch, W., Henze, M., Haberl, F., Hernanz, M., Sala, G., Hartmann, D. H., Della Valle, M. 2011, A\&A, 531, 22

Politano, M. 1996, ApJ, 456, 338

Rector, T. A. et al. 1999, BAAS, 195.3608

Ritchey, G. W. 1917, PASP, 29, 210

Rosino, L. 1964, AnAp, 27, 498.

Rosino, L. 1973, A\&AS, 9, 347.

Rosino, L., Capaccioli, M., D’Onofrio, M., della Valle, M. 1989, AJ, 87, 93.

Ruan, J. \& Gao, X. 2010a, CBET, \#2574

Ruan, J. \& Gao, X. 2010b, CBET, \#2594

Schaefer, B. E. 2010, ApJS, 187, 275

Schlegel, D. J., Finkbeiner, D. P., Davis, M. 1998, ApJ, 500, 525

Shafter, A. W. \& Irby, B. K. 2001, ApJ, 563, 749

Shafter, A. W. et al. 2008, ATel \#1851

Shafter, A. W., Bode, M. F., Darnley, M. J., Ciardullo, R., \& Misselt, K. A. 2010, ATel \#3006

Shafter, A. W., Bode, M. F., Darnley, M. J., Misselt, K. A., Rubin, M., Hornoch, K. 2011a, ApJ, 727, 50 
Shafter, A. W. et al. 2011b, ApJ, 734, 12

Shafter, A. W.; Hornoch, K.; Ciardullo, J. Vrastil R.; Darnley, M. J.; Bode, M. F. 2012a, ATel \#4503

Shafter, A. W., Hornoch, K., Ciardullo, R., Darnley, M. J., \& Bode, M. F. 2012b, ATel \#4658

Shafter, A. W., Darnley, M. J., Bode, M. F., Ciardullo, R. 2012c, ATel \#3877

Shafter, A. W., Darnley, M. J., Bode, M. F., Ciardullo, R., Schweizer, F. 2012d, ATel \#3914

Shafter, A. W., Hornoch, K., Ciardullo, R., Darnley, M. J., Bode, M. F. 2012e, ATel \#4368

Shafter, A. W., Curtin, C., Pritchet, C. J., Bode, M. F., Darnley, M. J. 2013, arXiv:1307.2296

Sharov, A. S. \& Alksnis, A. K. 1989, SvAL, 15, 382

Sharov, A. S. \& Alksnis, A. K. 1992, Ap\&SS, 190, 119

Sharov, A. S. \& Alksnis, A. 1997, AstL, 23, 540

Stratton, F. G. M. 1936, Handbuch der Astrophysik, 7, 671

Starrfield, S., Iliadis, C., \& Hix, R. 2008, in Classical Novae, 2nd ed., edited by M. Bode and A. Evans, Cambridge University Press, p. 77

Tang, S., Cao, Y., Kasliwal, M. M. 2013, ATel \#5607

Tang, S. 2014, ApJ, 786, 61

Townsley, D. M., Bildsten, L. 2005, ApJ, 628, 395

Warner, B. 2008, in Classical Novae, 2nd ed., edited by M. Bode and A. Evans, Cambridge University Press, p. 16

White, N. E., Giommi, P., Heise, J., Angelini, L., Fantasia, S. 1995, ApJ, 445, 125

Williams, R. E. 1992, AJ, 104, 725

Williams, B. F., Garcia, M. R., Kong, A. K. H., Primini, F. A., King, A. R., Di Stefano, R., Murray, S. S. 2004, ApJ, 609, 735

Williams, S. C., Darnley, M. J., Bode, M. F., Keen, A., \& Shafter, A. W. 2014, ApJS, 213, 10 
Williams, S. C. et al. 2015, in preparation

Wolf, William M., Bildsten, Lars, Brooks, Jared, Paxton, Bill 2013, ApJ, 777, 136

Yaron, O., Prialnik, D., Shara, M. M. \& Kovetz, A. 2005, ApJ, 623, 398

Yungelson, L., Livio, M., \& Tutukov, A. 1997, ApJ, 481, 127

Zheng, W. et al. 2010, CBET, 2574 


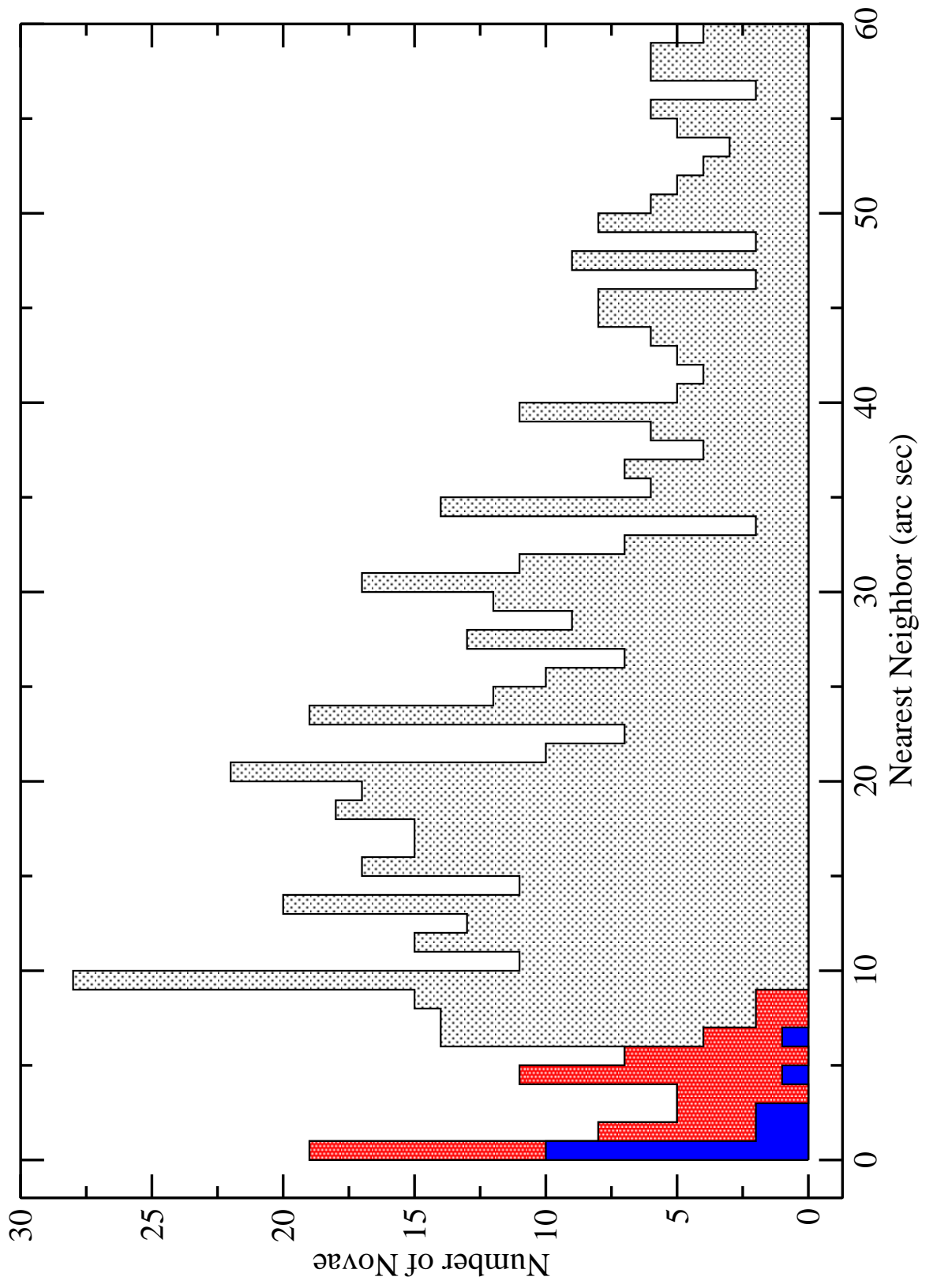

Fig. 1. - The nearest neighbor distribution for novae in M31. The angular separation between each of the 964 nova candidates in M31 and their nearest neighbor have been divided into $1^{\prime \prime}$ bins, and the total number of novae in each bin plotted as a function of angular separation. The shaded red region shows the novae identified in our initial screen for RNe, while the dark blue region represents systems that we have subsequently identified as RNe or likely RN systems. 

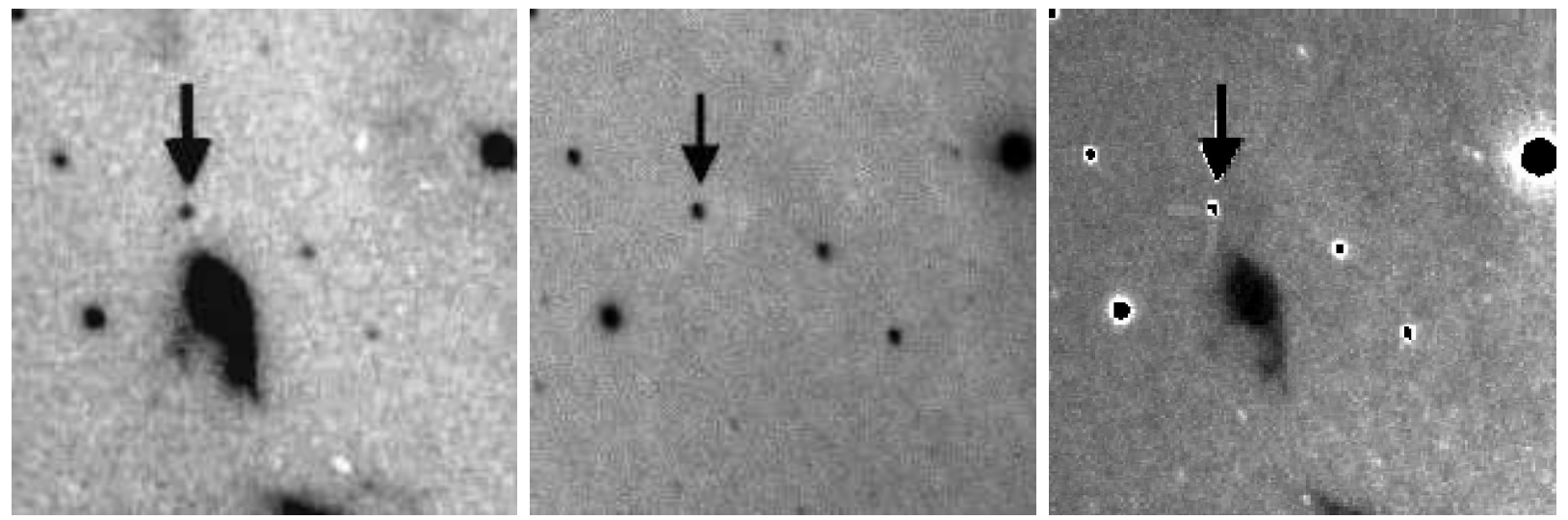

Fig. 2.- Images of M31N 1919-09a, 1998-06a, and their comparison (left, center, and right, respectively). The image for M31N 1919-09a is from plate \#5054 in the Carnegie archives, while that for 1998-06a is from the RBSE program at Kitt Peak National Observatory (Rector et al. 1999). The comparison image reveals that the two novae are spatially coincident to within the resolution afforded by the images $\left(\sim 1^{\prime \prime}\right)$. The black "smear" is part of ink marks on the glass side of the plate. North is up and East to the left, with a scale of $\sim 2^{\prime}$ on a side.
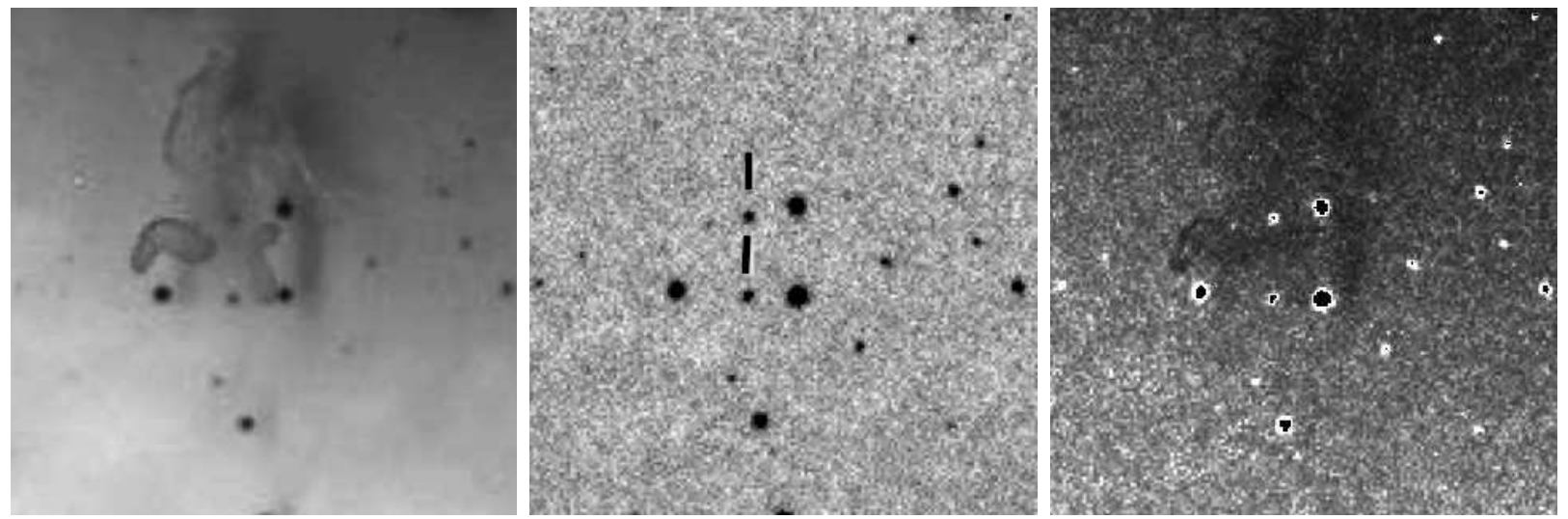

Fig. 3.- Images of M31N 1923-12c, 2012-01b, and their comparison (left, center, and right, respectively). The image for $\mathrm{M} 31 \mathrm{~N} 1923-12 \mathrm{c}$ is from plate $\mathrm{H} 348 \mathrm{H}$ in the Carnegie archives, while the chart of 2012-01b is courtesy of K. Nishiyama and F. Kabashima (Miyaki-Argenteus Observatory, Japan). As revealed by the comparison image, the novae are spatially coincident to within measurement uncertainties $\left(\sim 1^{\prime \prime}\right)$. North is up and East to the left, with a scale of $\sim 3^{\prime}$ on a side. 


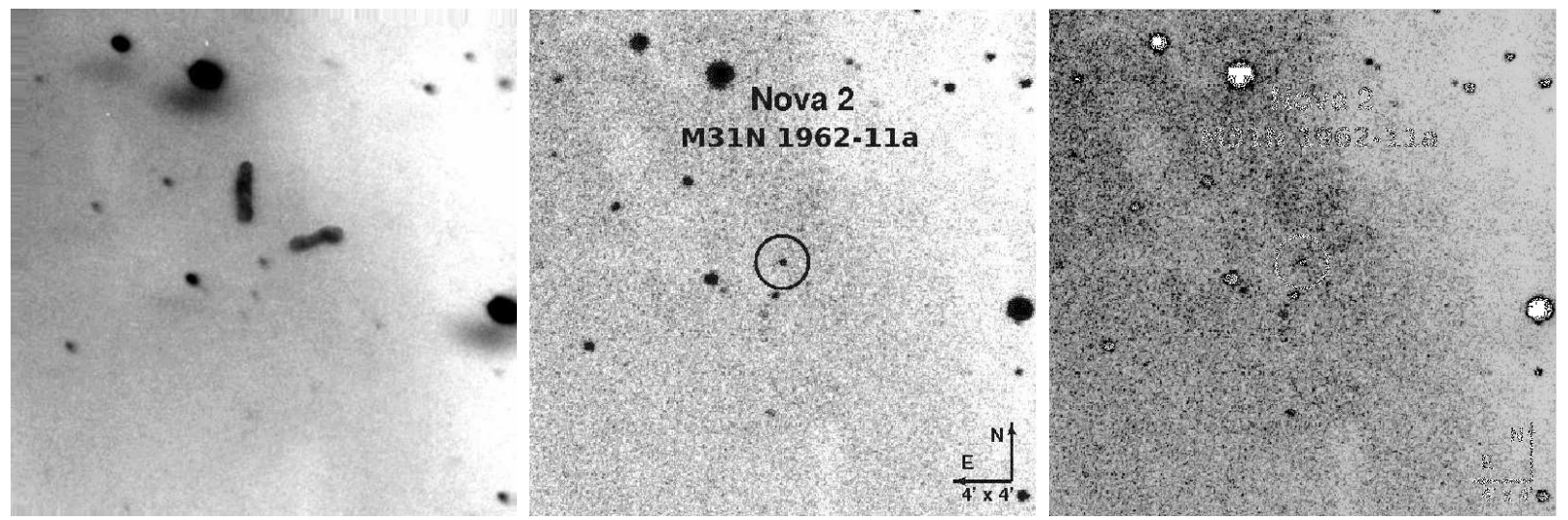

Fig. 4.- Images of M31N 1926-06a, 1962-11a, and their comparison (left, center, and right, respectively). The image for M31N 1926-06a was produced from plate H304D in the Carnegie archives, while the chart for 1962-11a is from Henze et al. (2008a). A comparison of the images reveals that the novae are spatially coincident to within measurement uncertainties $\left(\sim 1^{\prime \prime}\right)$. North is up and East to the left, with a scale of $\sim 4^{\prime}$ on a side.
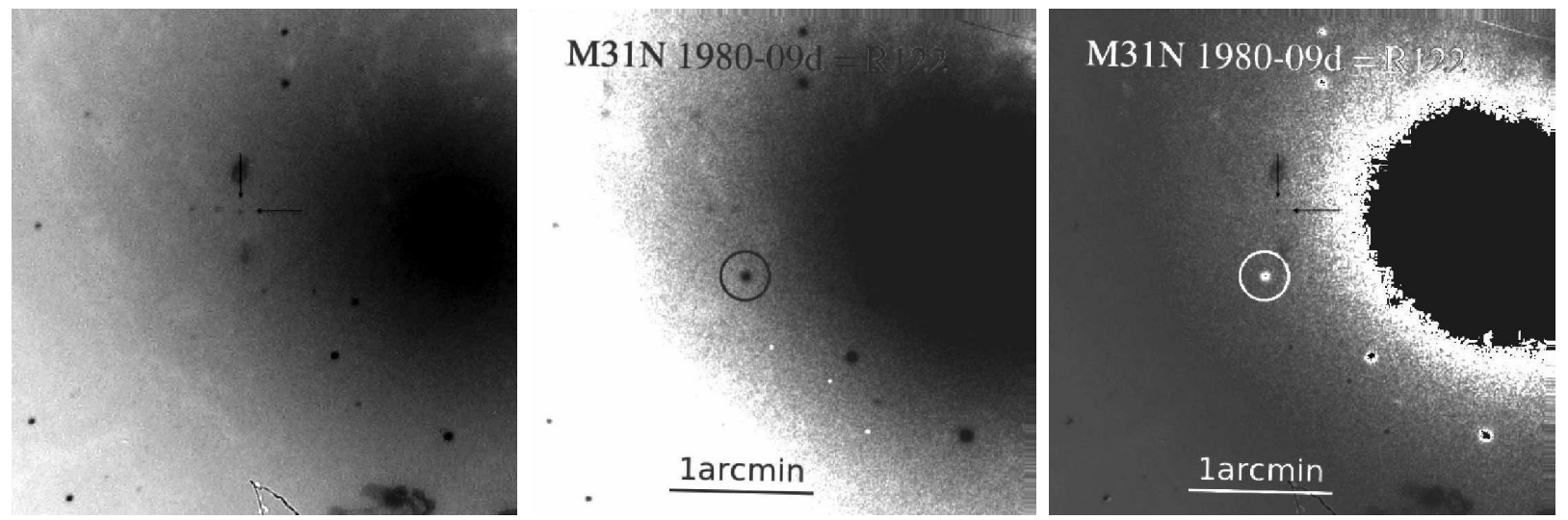

Fig. 5.- Images of M31N 1926-07c, 1980-09d and their comparison (left, center, and right, respectively). The comparison image shows that M31N 1980-09d (shown in white) is clearly not a recurrence of $1926-07 \mathrm{c}$, with the former nova being located $\sim 27^{\prime \prime}$ to the $\mathrm{S}$ of $1926-07 \mathrm{c}$. However, as seen in Figure6, M31N 1926-07c turns out to be coincident with M31N 1997-10f and 2008-08b, and thus is in fact a RN. The finding chart for M31N 1926-07c was reproduced from plate $\mathrm{H} 668 \mathrm{H}$ from the Carnegie archives, while the chart for 1980-09d is taken from the survey of Rosino et al. (1989). North is up and East to the left, with a scale of $\sim 3.5^{\prime}$ on a side. 

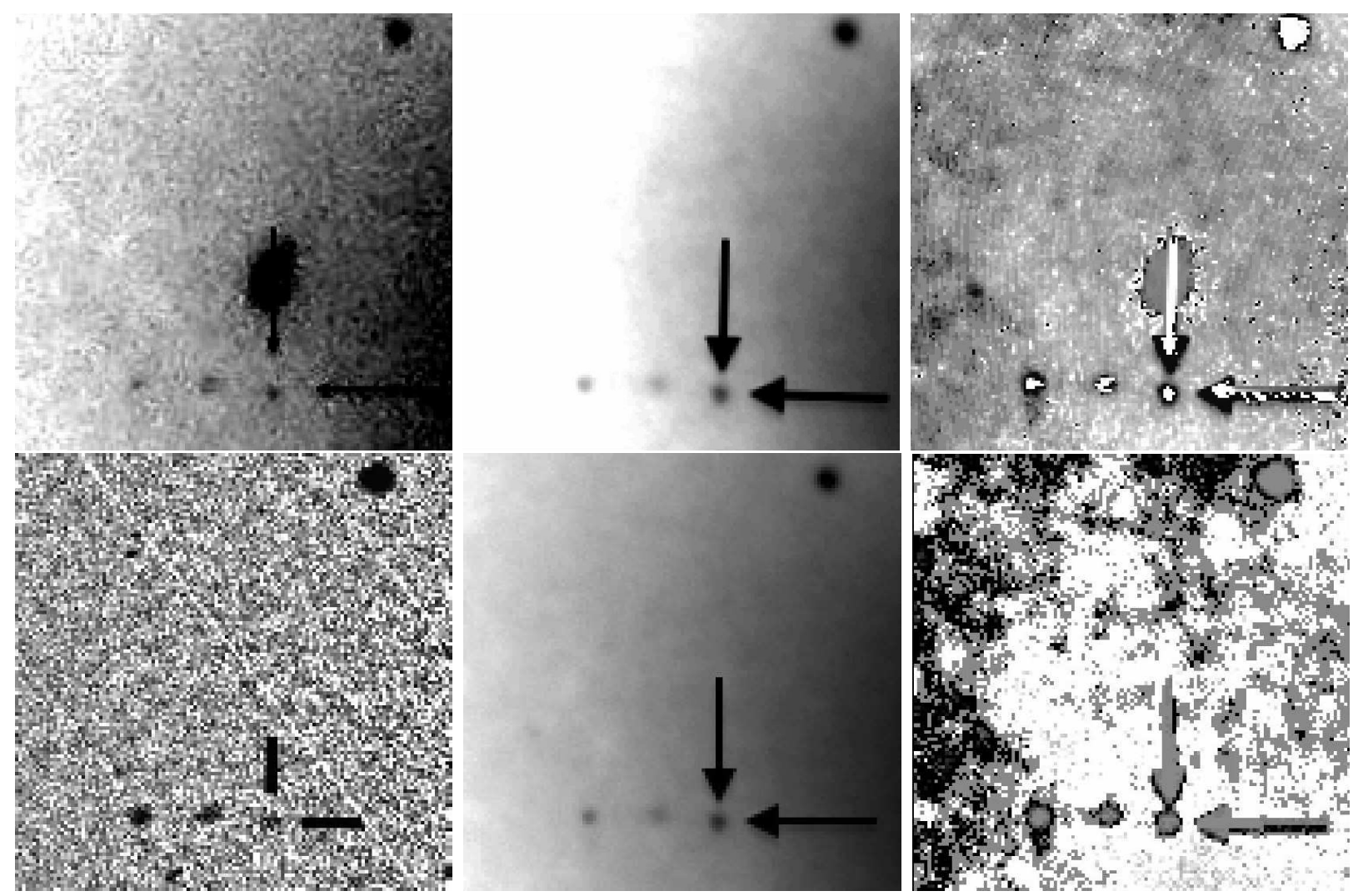

Fig. 6.- Images of M31N 1926-07c, 2008-08b, and their comparison (top left, center, and right), and M31N 1997-10f, 2008-08b, and their comparison (bottom left, center, and right, respectively). Although the novae erupted relatively close to the nucleus of M31, the comparison images reveal that the novae are all coincident to within measurement uncertainties estimated to be $\sim 1^{\prime \prime}$. Thus, M31N 1926-07c, although not coincident with 1980-09d (see Fig. 5), appears to be a RN clearly associated with both 1997-10f and 2008-08b. The chart for 1997-10f was produced from data taken in the survey of Shafter \& Irby (2001), while that for 2008-08b is taken from data reported in Henze et al. (2008a). North is up and East to the left, with a scale of $\sim 1^{\prime}$ on a side. 

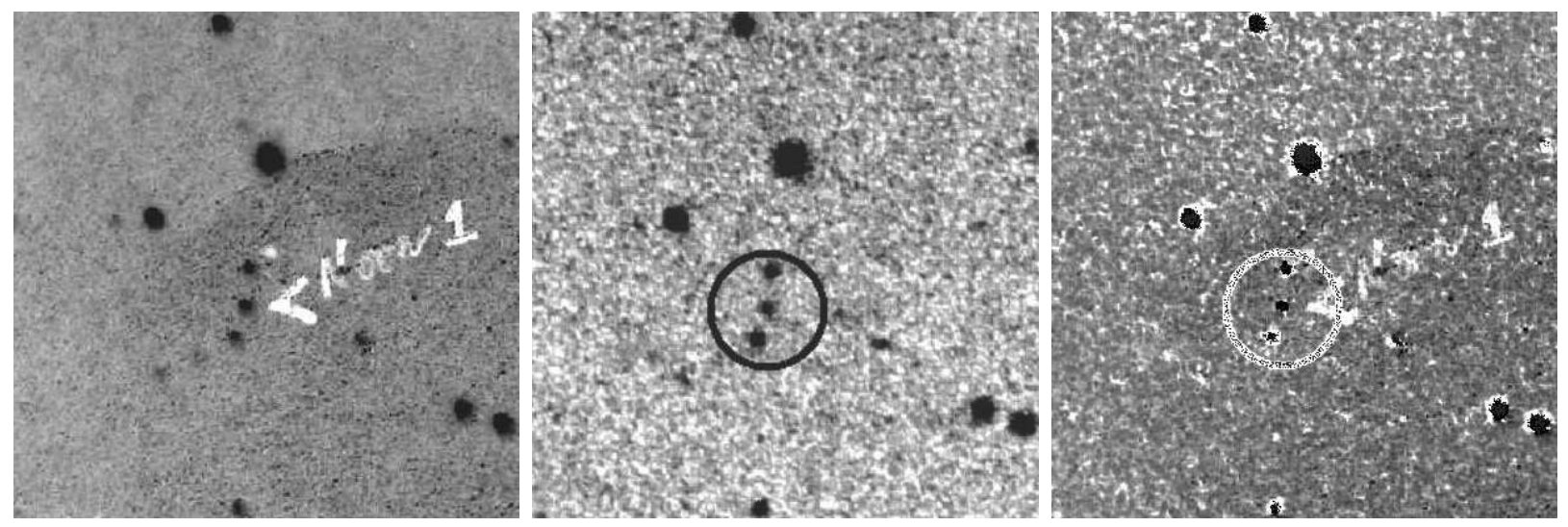

Fig. 7.- Images of M31N 1945-09c, 1975-11a, and their comparison (left, center, and right, respectively). The image for M31N 1945-09c is reproduced from print B1677B in the Huntington Library's Baade collection, while the chart for 1975-11a is taken from Henze et al. (2008a). The comparison image clearly establishes that the novae are spatially coincident to within less than an arcsec. North is up and East to the left, with a scale of $\sim 2^{\prime}$ on a side.
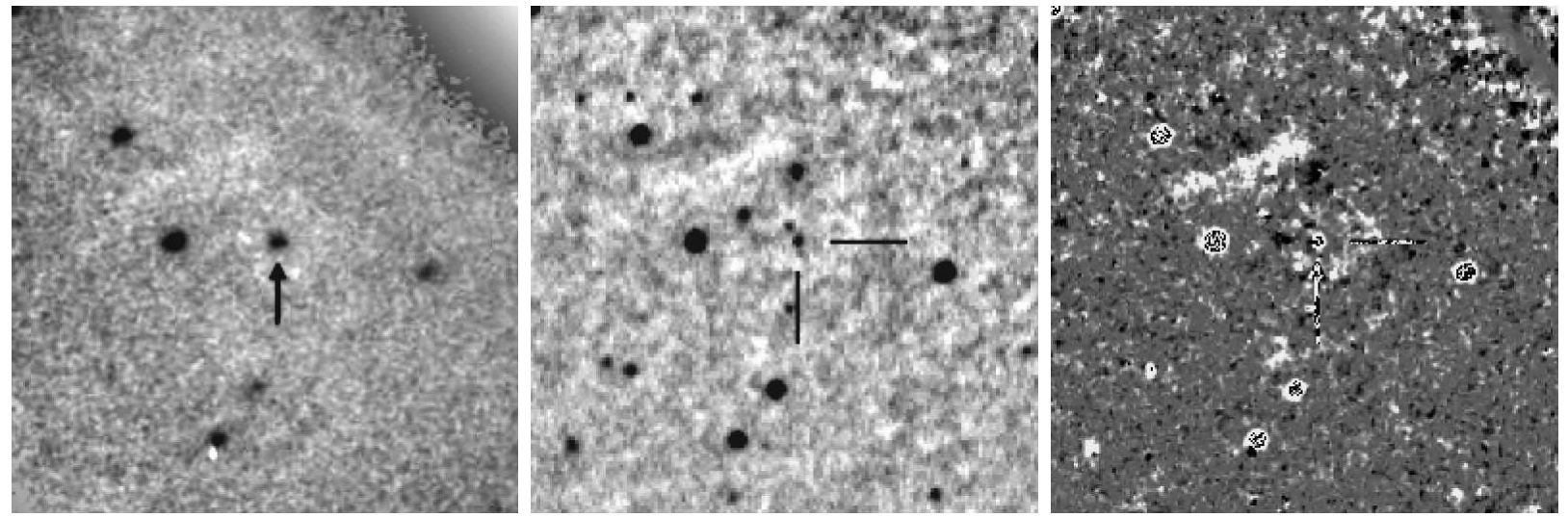

Fig. 8.- Images of M31N 1960-12a, 2013-05b, and their comparison (left, center, and right, respectively). The image for M31N 1960-12a was produced from data taken as part of the Rosino (1964) survey, while the chart for 2013-05b is from Hornoch et al. (2013). The comparison image establishes that the novae are spatially coincident to within measurement uncertainties $\left(\sim 1^{\prime \prime}\right)$. North is up and East to the left, with a scale of $\sim 2.5^{\prime}$ on a side. 

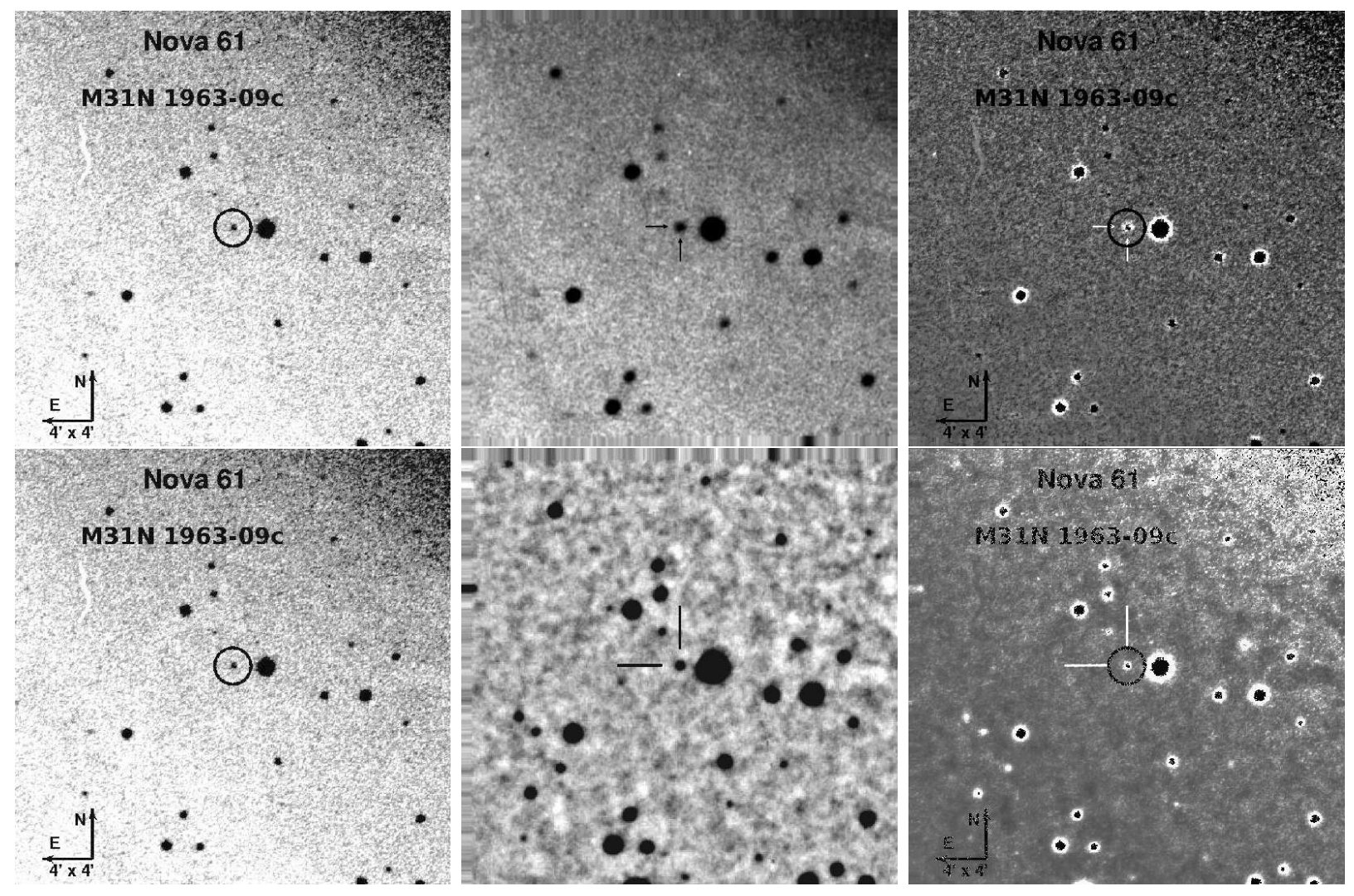

Fig. 9.- Images of M31N 1963-09c, 1968-09a, and their comparison (top left, center, and right), and M31N 1963-09c, 2010-10e, and their comparison (bottom left, center, and right, respectively). The images for M31N 1963-09c, 1968-09a and 2010-10e are from Henze et al. (2008a), Rosino (1973) and Hornoch et al. (2010), respectively. As is clear from the comparison images M31N 1963-09c, 1968-09a and 2010-10e are spatially coincident to within measurement uncertainties $\left(\sim 1^{\prime \prime}\right)$. North is up and East to the left, with a scale of $\sim 4^{\prime}$ on a side. 

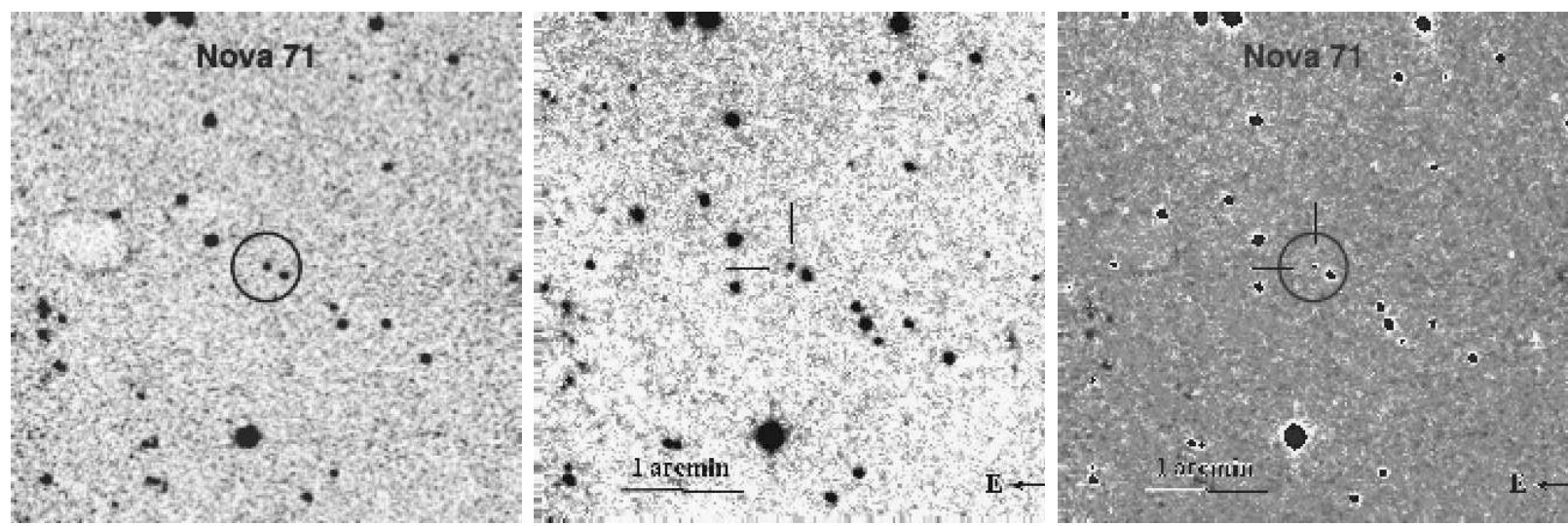

Fig. 10.- Images of M31N 1966-09e, 2007-08d, and their comparison (left, center, and right, respectively). The chart for M31N 1966-09e is from Henze et al. (2008a), while that for 2007-08d is from Pietsch et al. (2007b). The comparison image established that the novae are spatially coincident to within $\sim 1^{\prime \prime}$. North is up and East to the left, with a scale of $\sim 4^{\prime}$ on a side.
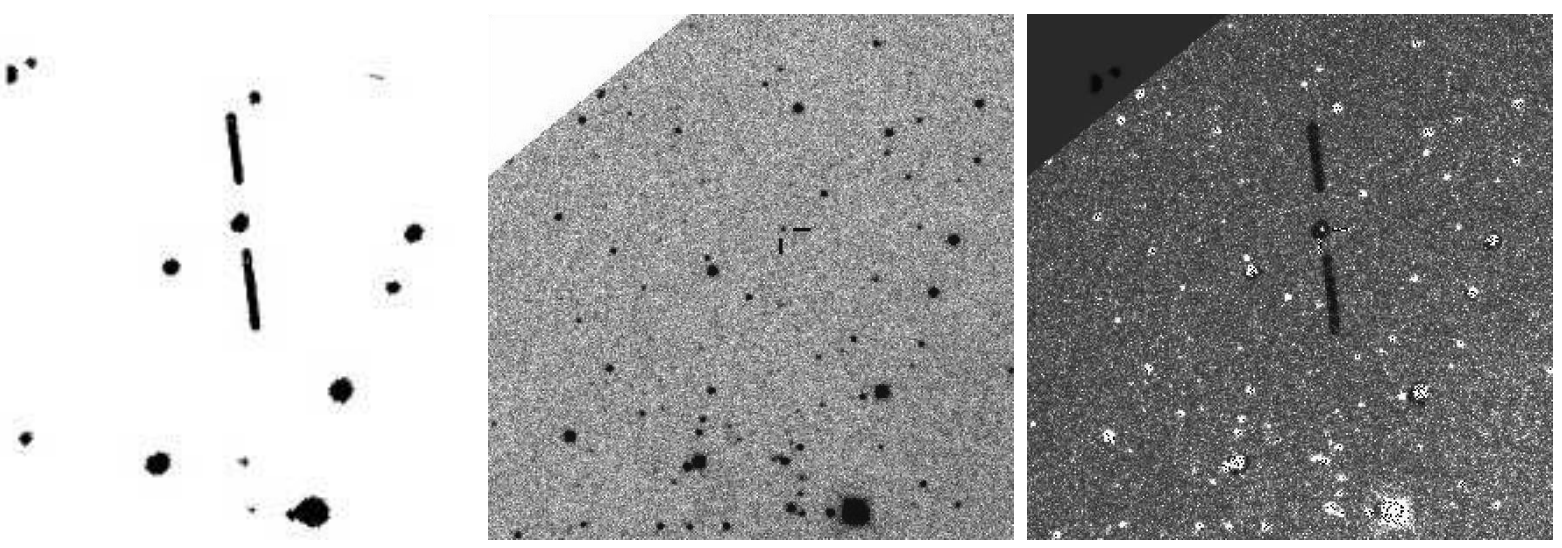

Fig. 11.- Images of M31N 1982-08b, 1996-08c, and their comparison (left, center, and right, respectively). The chart for M31N 1982-08b is taken from Sharov \& Alksnis (1992), with that for 1996-08c taken from the survey of Shafter \& Irby (2001). Although the finding chart for M31N 1982-08b has poor spatial resolution, the novae appear spatially coincident to within measurement uncertainties $\left(\sim 2^{\prime \prime}\right)$. At the location of the novae in the outskirts of M31 $\left(a \sim 60^{\prime}\right)$, the probability of a chance positional coincidence is negligible. North is up and East to the left, with a scale of $\sim 5^{\prime}$ on a side. 

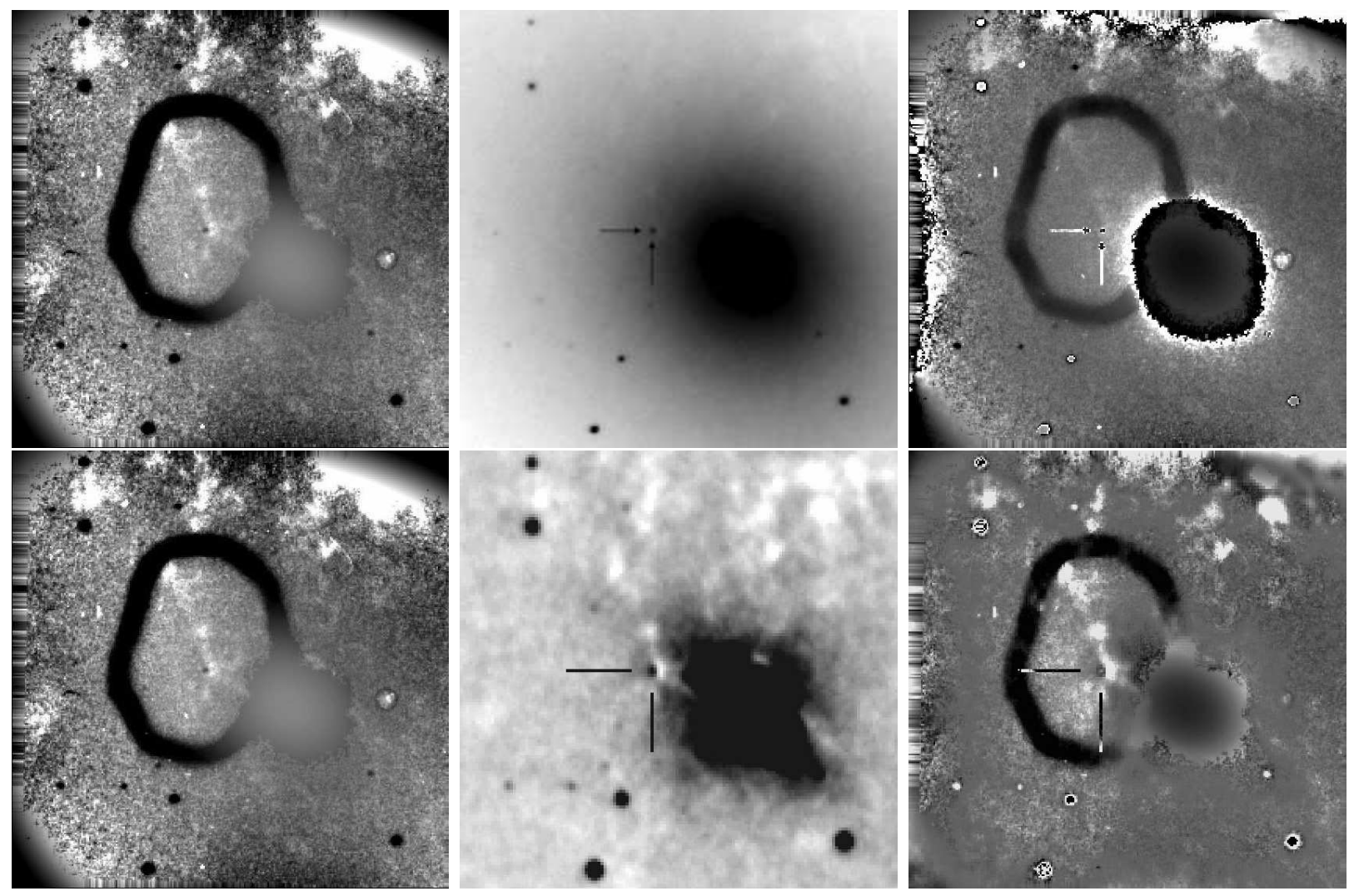

Fig. 12.- Images of M31N 1984-07a, 2004-11f and their comparison (top left, center, and right), and M31N 1984-07a, 2012-09a, and their comparison (bottom left, center, and right, respectively). The chart for M31N 1984-07a is from the survey of Rosino et al. (1989), that for 2004-11f is from the RBSE project (Rector et al. 1999), and that for 2012-09a is from the data reported in Hornoch \& Vrastil (2012a). Although the novae erupted very close to the nucleus of M31 making a detailed comparison of the positions challenging, the comparison images show that the novae appear to be spatially coincident to less than 1 arcsec. North is up and East to the left, with a scale of $\sim 2.5^{\prime}$ on a side. 

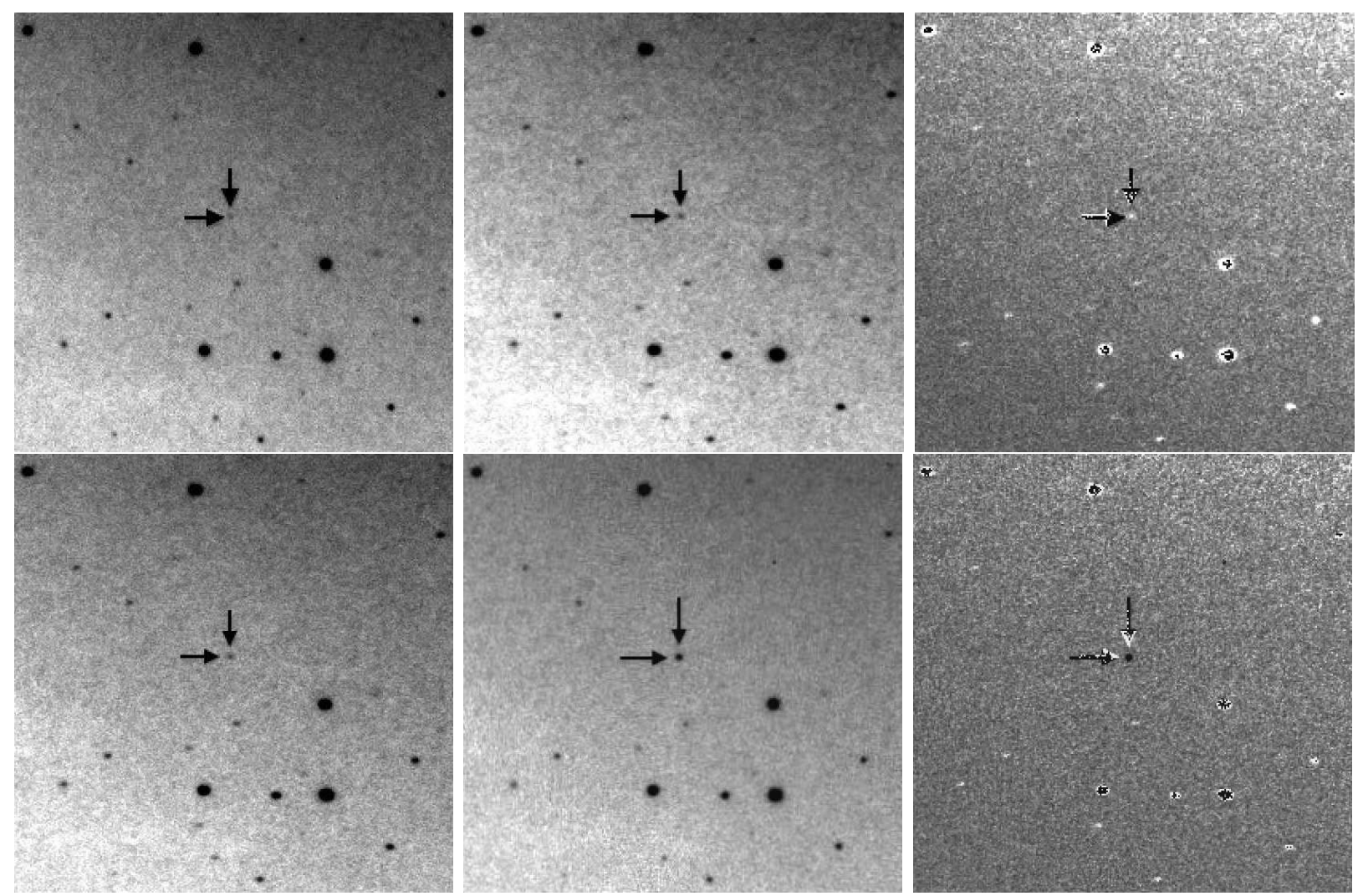

Fig. 13.- Images of M31N 1997-11k, 2001-12b and their comparison (top left, center, and right), and M31N 2001-12b, 2009-11b and their comparison (bottom left, center, and right, respectively). Charts for all three novae are taken from images obtained as part of the RBSE program at KPNO (Rector et al. 1999). Although, not shown clearly in the comparison image (due to the dissimilar brightness of the novae at the time the images were obtained), a careful inspection of the positions reveal that all three novae are spatially coincident to within the estimated measurement uncertainties of $\sim 1^{\prime \prime}$. North is up and East to the left, with a scale of $\sim 2.8^{\prime}$ on a side. 

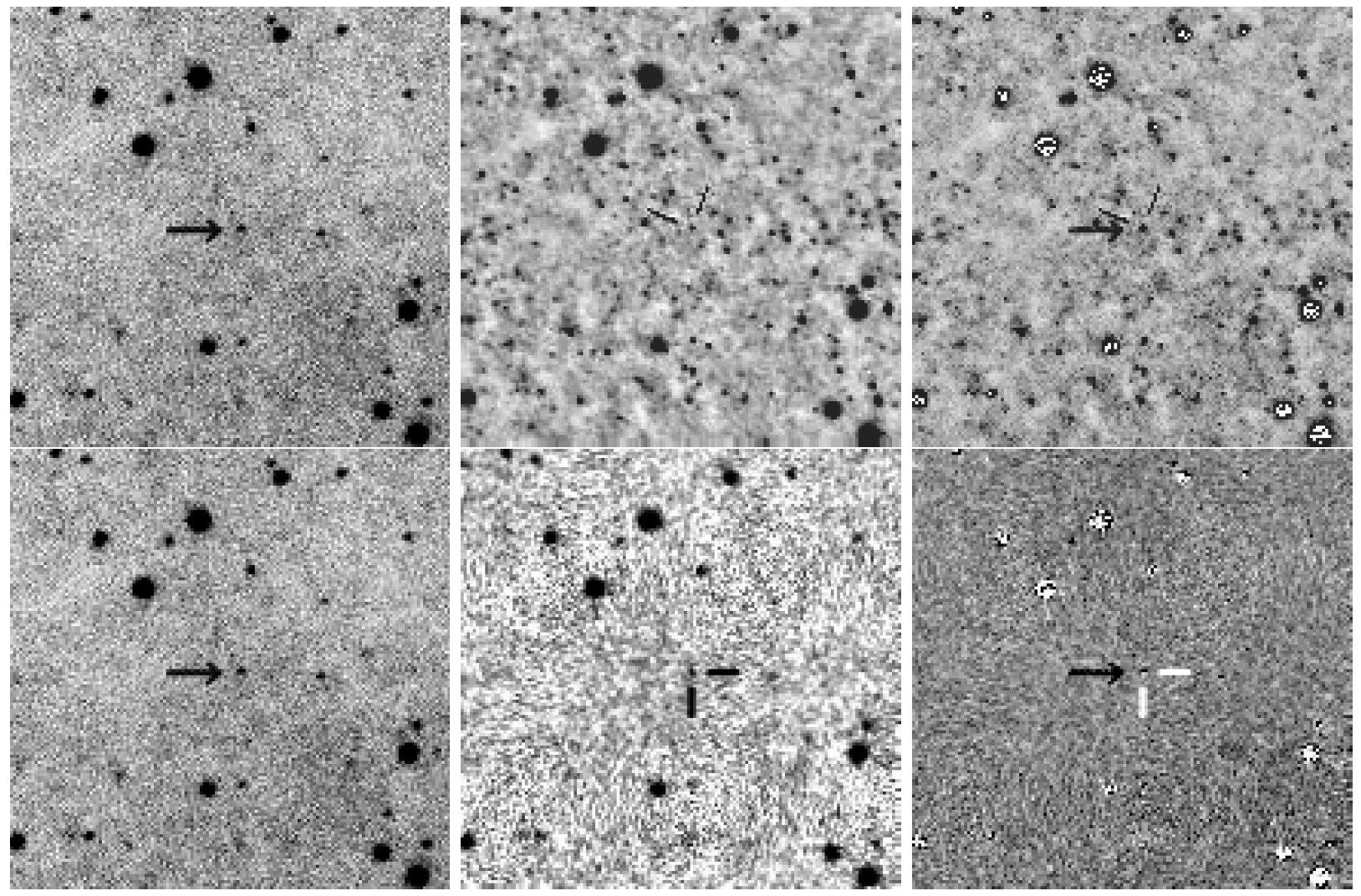

Fig. 14.- Images of M31N 2008-12a, 2011-10e and their comparison (top left, center, and right), and M31N 2008-12a, M31N 2012-10a, and their comparison (bottom left, center, and right, respectively). The charts for 2008-12a and 2012-10a are courtesy of K. Nishiyama and F. Kabashima (Miyaki-Argenteus Observatory, Japan), while that for 2011-10e is from (Barsukova et al. 2011). Although not obvious from the comparison images, a careful inspection of the positions of the novae show that all three are in fact spatially coincident to within measurement uncertainties of $\sim 1^{\prime \prime}$. At the nova's position in the outskirts of M31, the probability of a chance positional coincidence is negligible. North is up and East to the left, with a scale of $\sim 3^{\prime}$ on a side. 

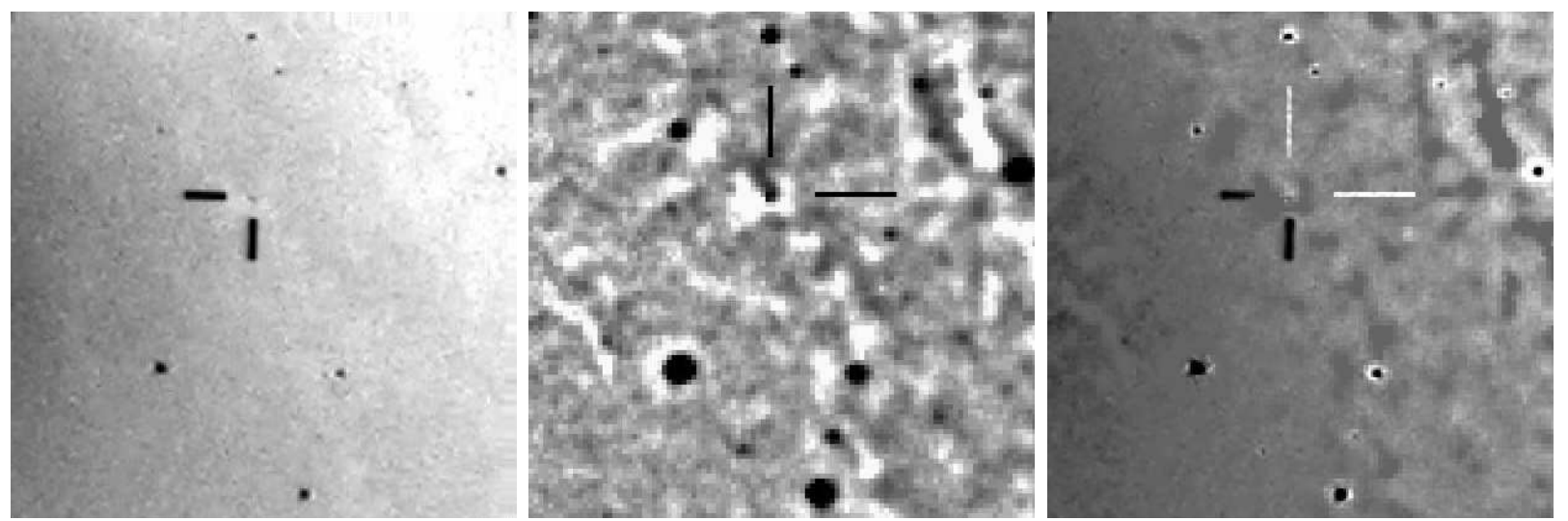

Fig. 15.- Images of M31N 1953-09b, 2004-08a, and their comparison (left, center, and right, respectively). The image of M31N 1953-09b is a scan of the plate S967A from Arp (1956), while that of 2004-08a is from K. Hornoch (2004, unpublished). The comparison image suggests that M31N 1953-09b may be slightly south of the position of 2004-08a, but given the measurement uncertainties, the objects are possibly coincident. North is up and East to the left, with a scale of $\sim 3.5^{\prime}$ on a side.
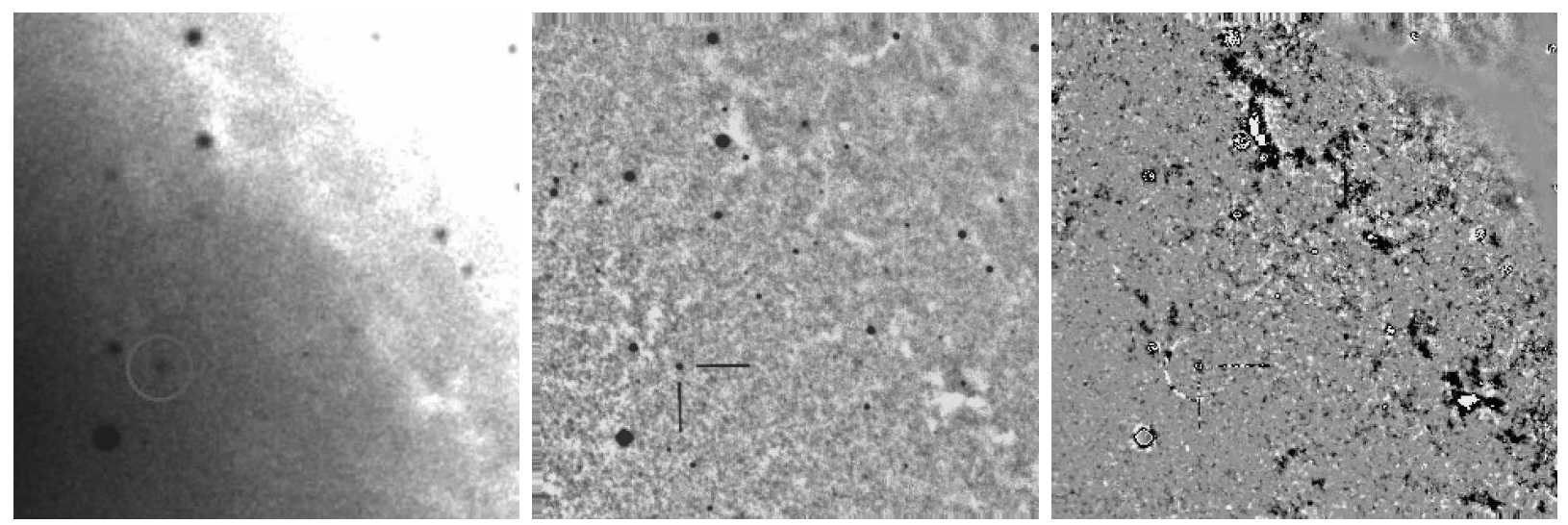

Fig. 16.- Images of M31N 1961-11a, 2005-06c, and their comparison (left, center, and right, respectively). The image of M31N 1961-11a has been produced from data taken as part of the Rosino (1964, 1973) survey, while that of 2005-06c is from K. Hornoch (previously unpublished). The comparison image confirms that the novae are coincident to within measurement uncertainties $\left(\sim 1^{\prime \prime}\right)$. North is up and East to the left, with a scale of $\sim 3.5^{\prime}$ on a side. 

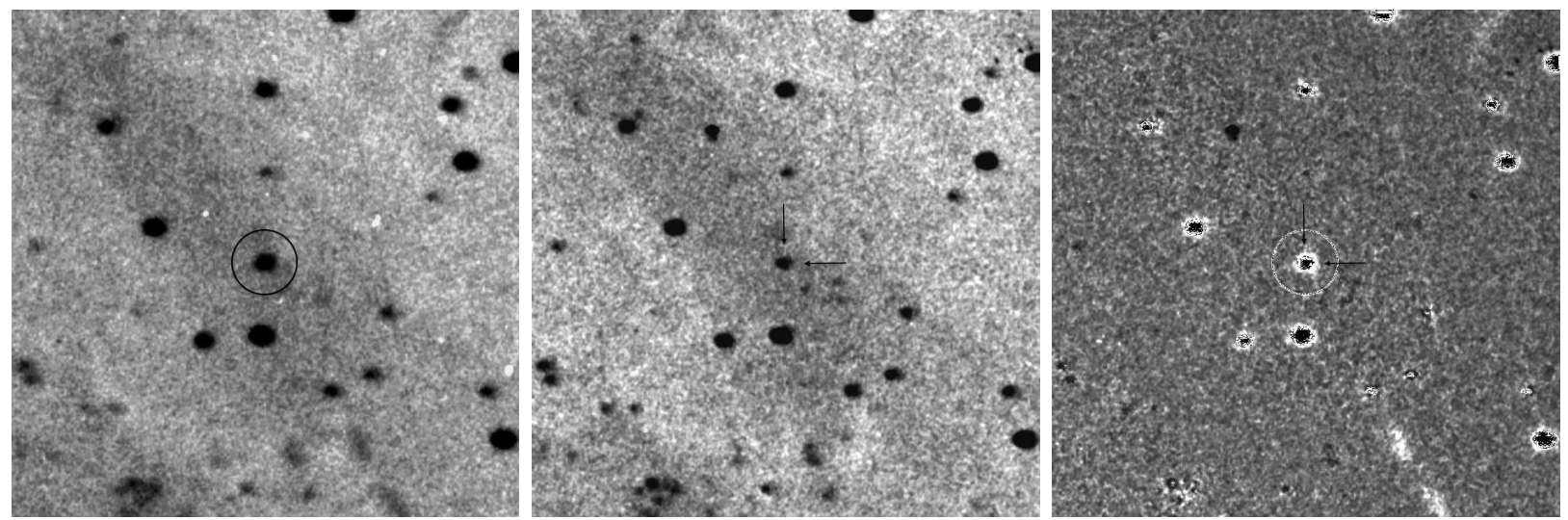

Fig. 17.- Images of M31N 1966-08a, 1968-10c, and their comparison (left, center, and right, respectively). Both images are from the survey of Rosino (1973). The comparison image confirms that both novae are spatially coincident. North is up and East to the left, with a scale of $\sim 4^{\prime}$ on a side.
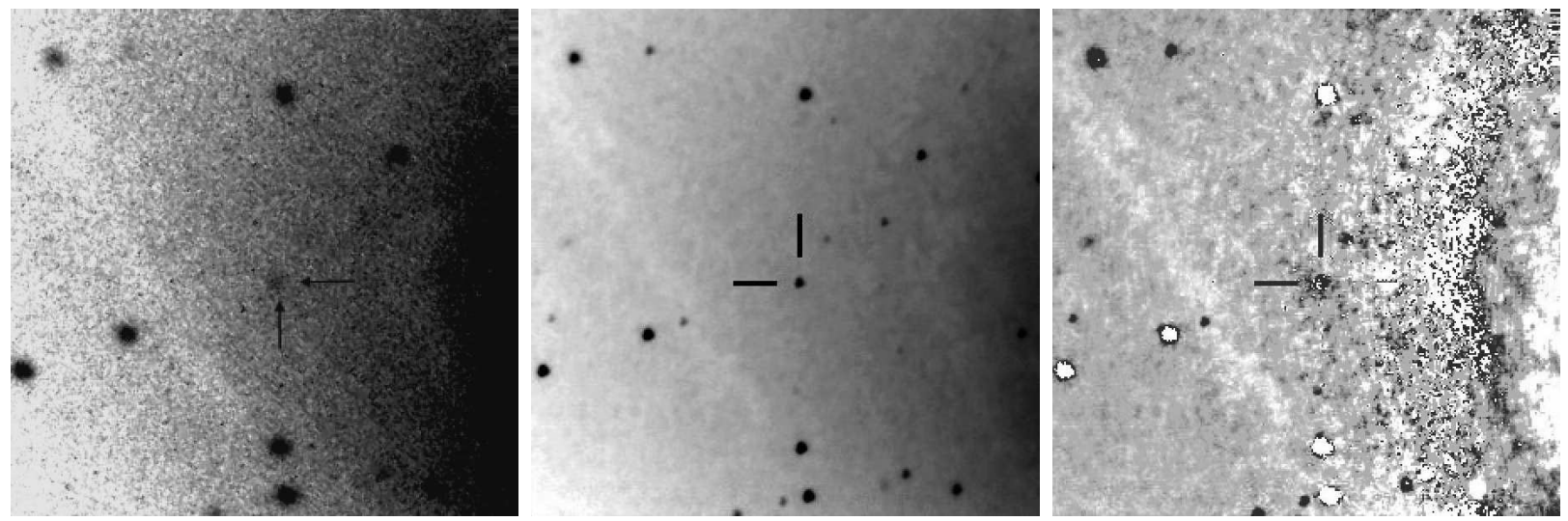

Fig. 18. - Images of M31N 1990-10a, 2007-07a, and their comparison (left, center, and right, respectively). The chart for M31N 1990-10a is based on a 35-mm photographic negative obtained by one of us (J. B.) on 1990 Oct 13.16, while that for 2007-07a is taken from observations at the Skinakas Observatory (Hatzidimitriou et al. 2007a). M31N 2007-07a is coincident to within $\sim 1^{\prime \prime}$ of the position of M31N 1990-10a, although it appears that the former nova may be just slightly NW of 1990-10a, and the relatively poor image quality of the photographic negative does not allow us to make a definitive judgment as to whether the two novae are in fact spatially coincident. North is up and East to the left, with a scale of $\sim 2.5^{\prime}$ on a side. 

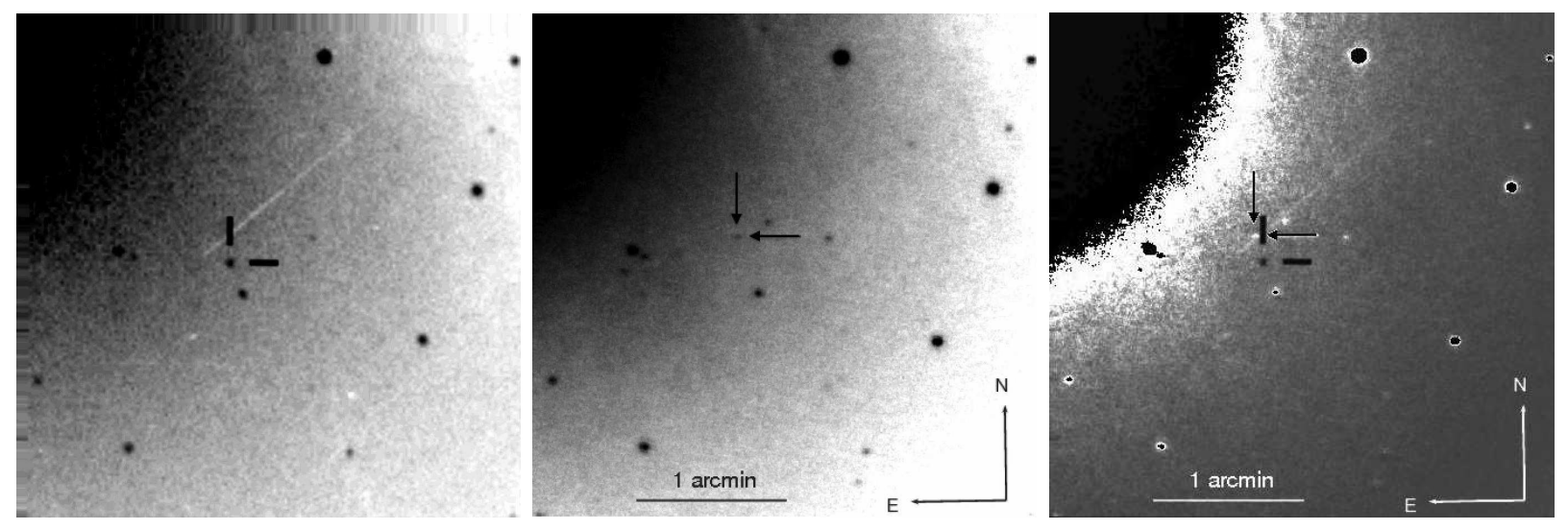

Fig. 19.- Images of M31N 1909-09b, M31N 2009-02b, and their comparison (left, center, and right, respectively). The comparison image shows that the positions of the two novae are clearly not coincident, with M31N 2009-02b (in white) being located 10.5" NNE of the position of 1909-09b. The image for M31N 1909-09b is a reproduction of plate S19-Ri from the Carnegie archives, while that for 2009-02b is from the SuperLOTIS project (Pietsch et al. 2009a). North is up and East to the left, with a scale of $\sim 3.4^{\prime}$ on a side.
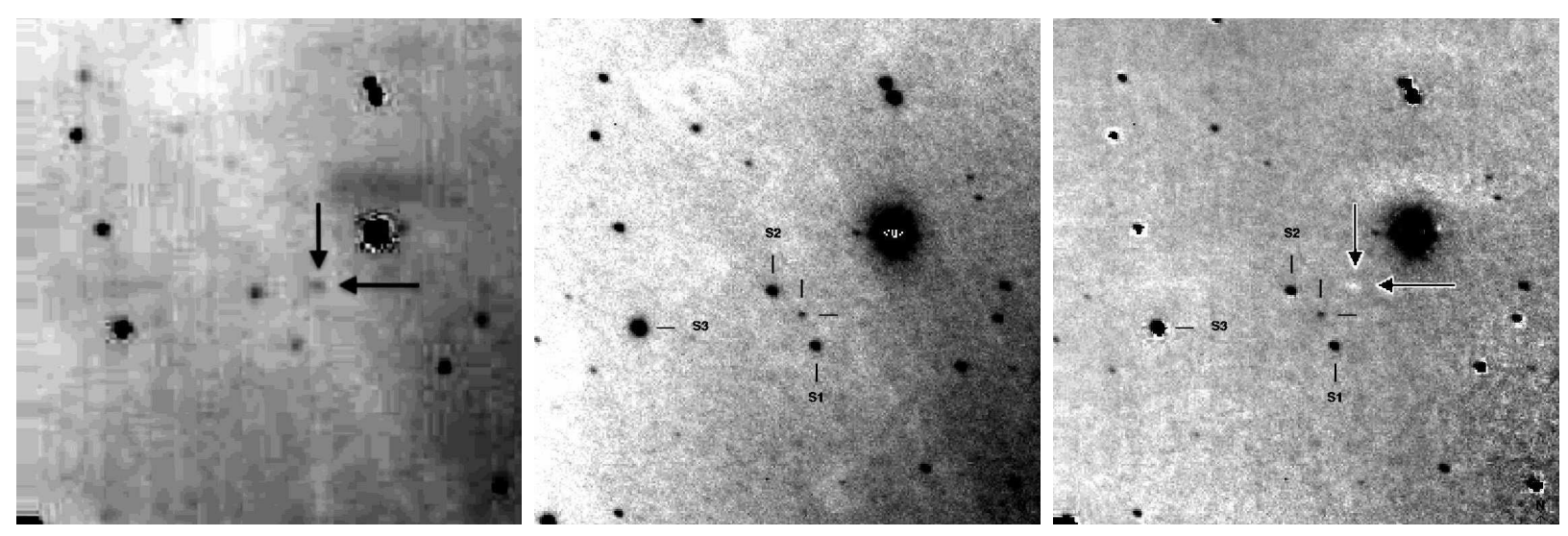

Fig. 20.- Images of M31N 1918-02b, M31N 2013-10g, and their comparison (left, center, and right, respectively). The comparison image reveals that the two novae are clearly not spatially coincident, with M31N 1918-02b (in white) being located $\sim 18^{\prime \prime}$ NW of the position of 2013-10g. The image for M31N 1918-02b is a reproduction of plate S162-Ri from the Carnegie archives, while that of 2013-10g is from the Lick Observatory Supernova Search. North is up and East to the left, with a scale of $\sim 3.5^{\prime}$ on a side. 

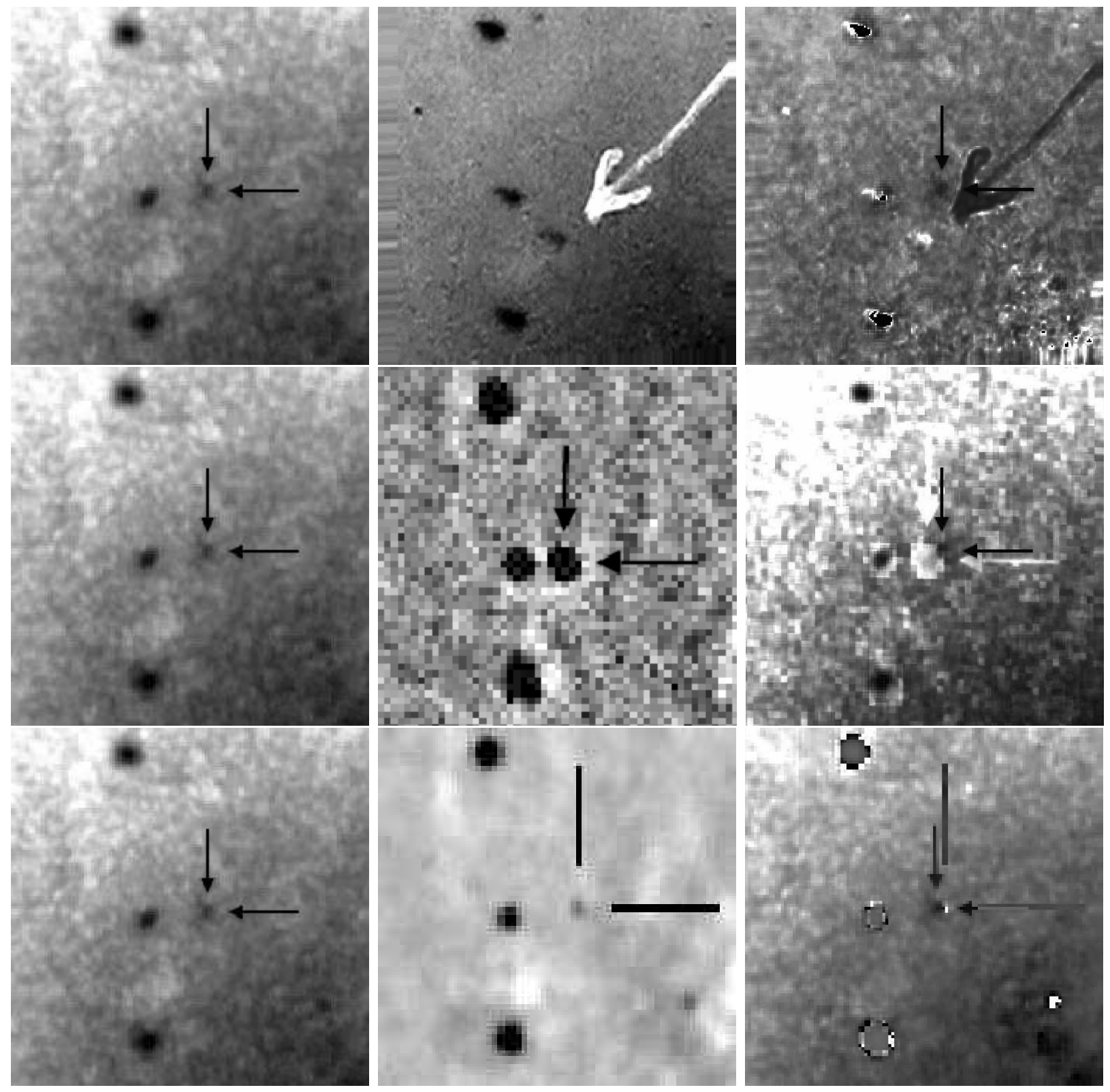

Fig. 21. - Images of M31N 1967-12a, 1923-02a, and their comparison (top left, center, and right), M31N 1967-12a, 1993-11c and their comparison (middle left, center, and right), and M31N 1967-12a, 2013-08b, and their comparison (bottom left, center, and right, respectively). M31N 1923-02a, 1967-12a, 1993-11c and 2013-08b are clearly not coincident with one another; however, M31N 1967-12a and 2013-08b appear quite close. Despite their proximity, precise astrometry and the comparison image reveal that the novae are not coincident, with M31N 2013-08b (in white) being 2" West of the position of 1967-12a. The image for M31N1923-02a was produced from a photograph of plate A47 in the Carnegie archives taken on 1923 Feb 15. The charts for M31N 1967-12a and 1993-11c are taken from unpublished data from the surveys of Rosino (1973) and Shafter \& Irby (2001), with the chart for 201308b taken from Hornoch \& Vrastil (2013). North is up and East to the left, with a scale of $\sim 1^{\prime}$ on a side. 

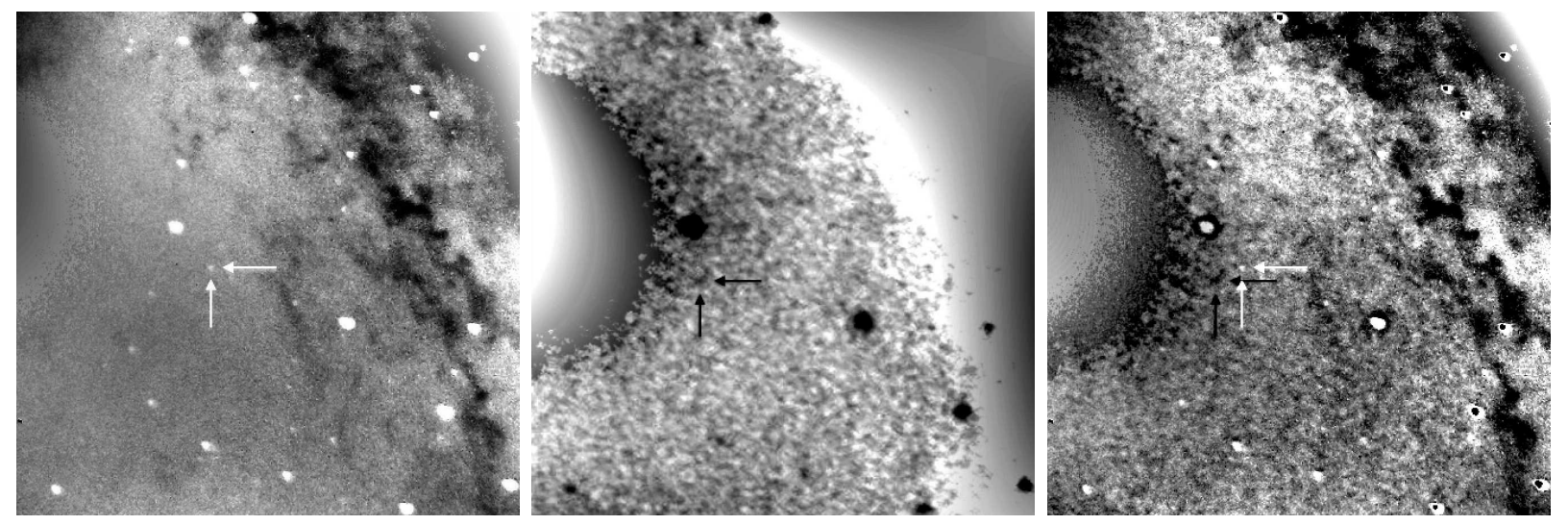

Fig. 22.- Images of M31N 1924-08a, M31N 1987-12a, and their comparison (left, center, and right, respectively). The comparison image reveals that the two novae are clearly not spatially coincident, with M31N 1987-12a (in black) being 20" SE of the position of 192408a. The image for M31N 1924-08a is a reproduction from plate H246D from the Carnegie archives, while that of 1987-12a is from a 35mm negative obtained by one of us (JB) on 1987 Dec. 20.13 UT (Bryan 1987). North is up and East to the left, with a scale of $\sim 5^{\prime}$ on a side.
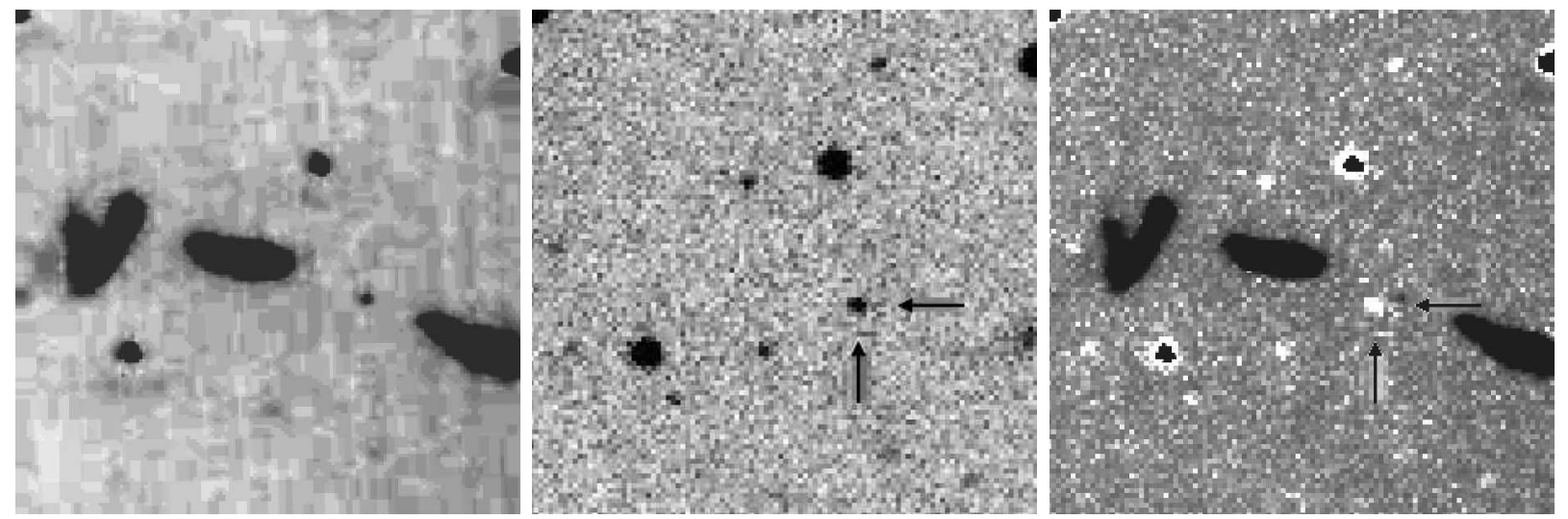

Fig. 23.- Images of M31N 1925-07c, 2011-12b, and their comparison (left, center, and right, respectively). As is clear from the comparison image, the recent outburst M31N 2011-12b (in white) erupted $\sim 6.6^{\prime \prime}$ ESE of the position of 1925-07c, and thus is not a recurrence. The image of M31N 1925-07c has been reproduced from plate $\mathrm{H} 580 \mathrm{H}$ in the Carnegie archives, while the image for 2011-12b is courtesy of K. Nishiyama of the MiyakiArgenteus Observatory. North is up and East to the left, with a scale of $\sim 2.2^{\prime}$ on a side. 

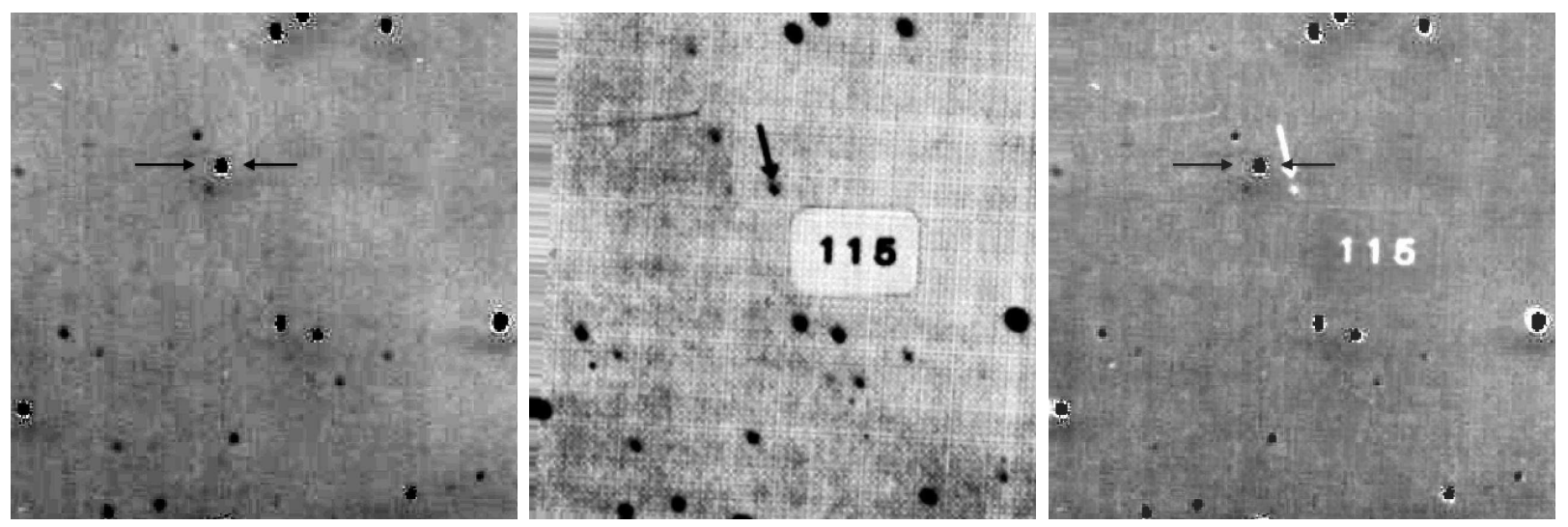

Fig. 24.- Images of M31N 1925-09a, 1976-12a and their comparison (left, center, and right, respectively). The comparison image shows that M31N 1976-12a is clearly not a recurrence of 1925-09a (shown in white), with the former nova being located $\sim 15^{\prime \prime}$ to the SW of 192509a. The finding chart for M31N 1925-09a has been reproduced from plate H609H from the Carnegie archives, while the chart for 1976-12a is taken from Rosino et al. (1989). North is up and East to the left, with a scale of $\sim 3.2^{\prime}$ on a side.
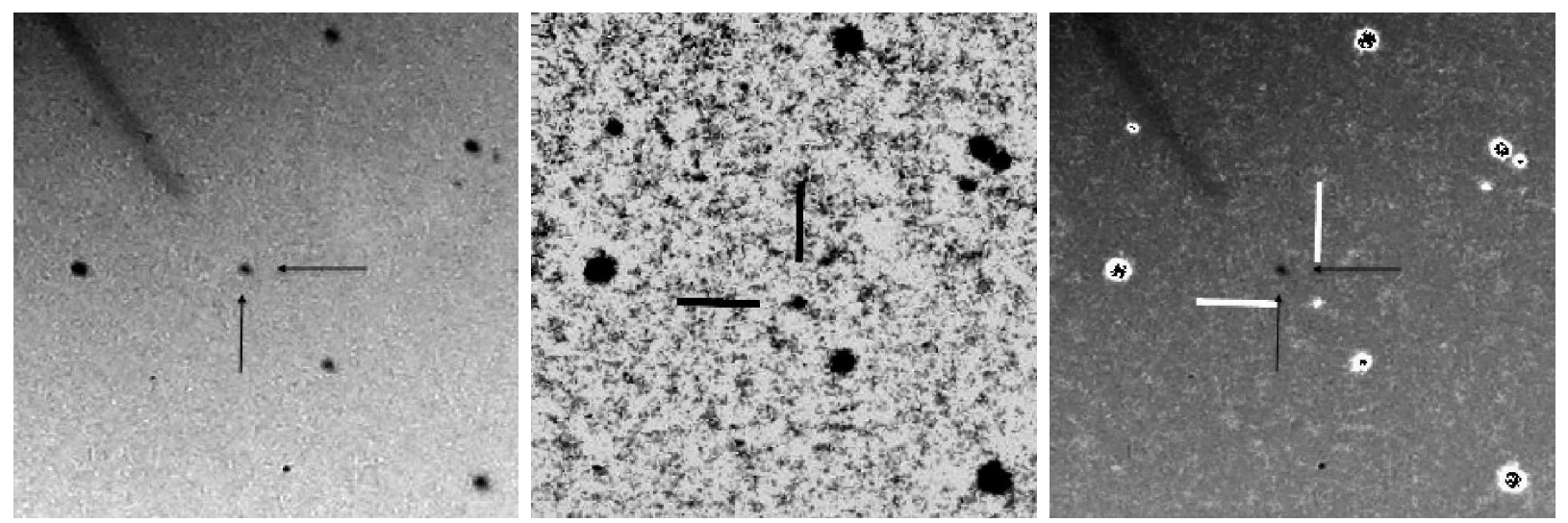

Fig. 25.- Images of M31N 1927-08a, 2009-11e and their comparison (left, center, and right, respectively). The comparison image shows that M31N 2009-11e (shown in white) is clearly not a recurrence of 1927-08a, with the former nova being located $\sim 20^{\prime \prime}$ to the SW of 192708a. The finding chart for M31N 1927-08a was reproduced from plate H828H from the Carnegie archives, while the chart for 2009-11e is taken from SuperLOTIS project (Burwitz et al. 2010). North is up and East to the left, with a scale of $\sim 2.3^{\prime}$ on a side. 

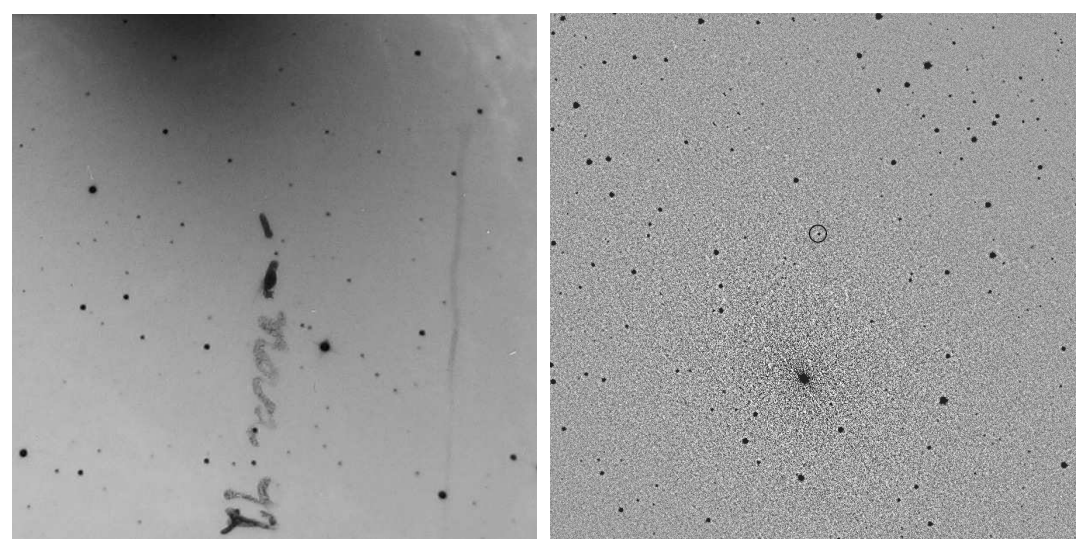

Fig. 26. - Images of M31N 1930-06c and 1996-08a (left and right, respectively). A comparison of the position of M31N 1930-06c (Hubble's nova \#92) with that of 1996-08a shows that the novae lie on opposite sides of the nucleus of M31, and are clearly distinct objects. North is up and East to the left, with a scale of $\sim 8^{\prime}$ on a side.
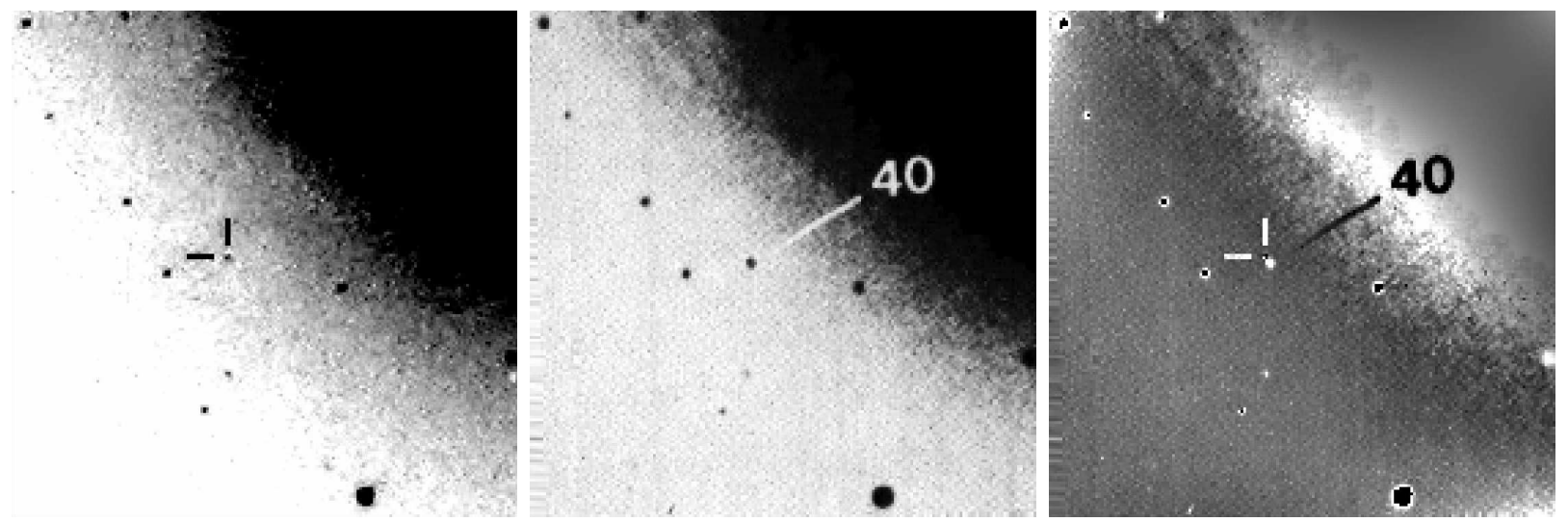

Fig. 27.- Images of M31N 1953-11a, 1962-11b and their comparison (left, center, and right, respectively). A careful comparison afforded by the comparison image shows that M31N 1962-11b (in white) is located slightly SW of the position of 1953-11a, establishing that that the former nova is not a recurrence of the latter. The image of M31N 1953-11a is a scan of the plate S1137A from Arp (1956), while that of 1962-11b is from Rosino (1973). North is up and East to the left, with a scale of $\sim 3.5^{\prime}$ on a side. 


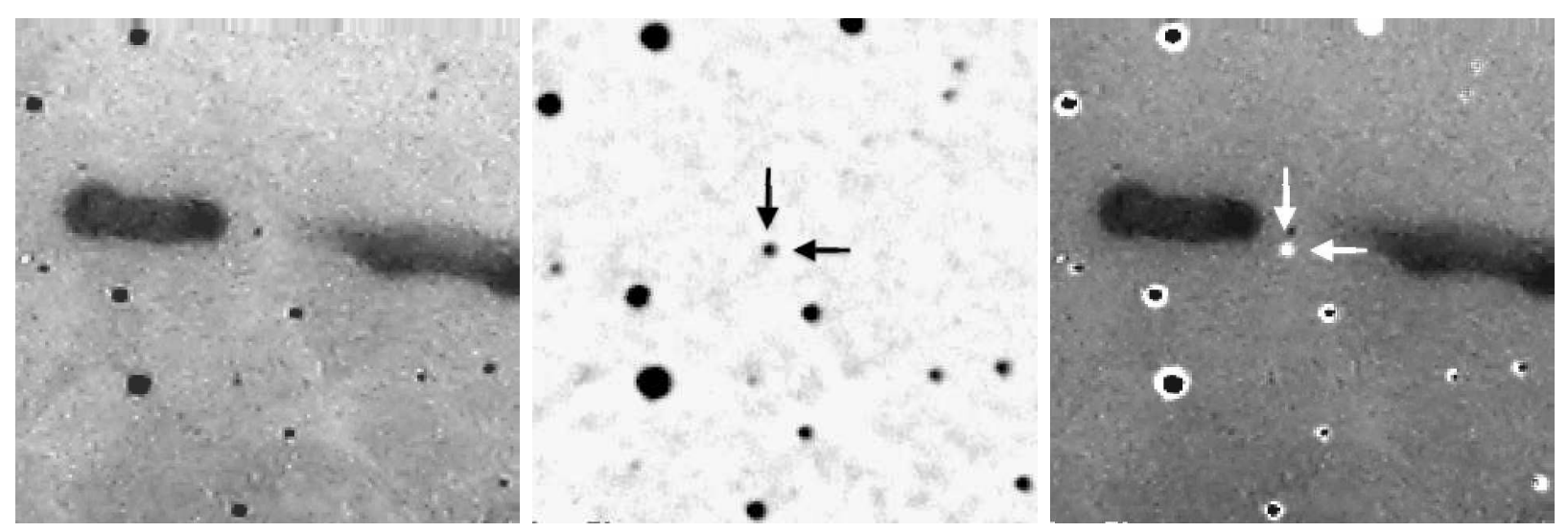

Fig. 28.- Images of M31N 1954-06c, 2010-01b and their comparison (left, center, and right, respectively). M31N 2010-01b (in white) is located several arcsec to the SSE of 1954-06c and is clearly not spatially coincident with it. The image of M31N 1954-06c is a scan of the plate S1335A from Arp (1956), while that of 2010-01b is a previously unpublished finding chart based on data obtained by V. Burwitz using the TOU/OAM PIRATE Schmidt-Cassegrain telescope at Costitx, Mallorca. North is up and East to the left, with a scale of $\sim 3^{\prime}$ on a side.
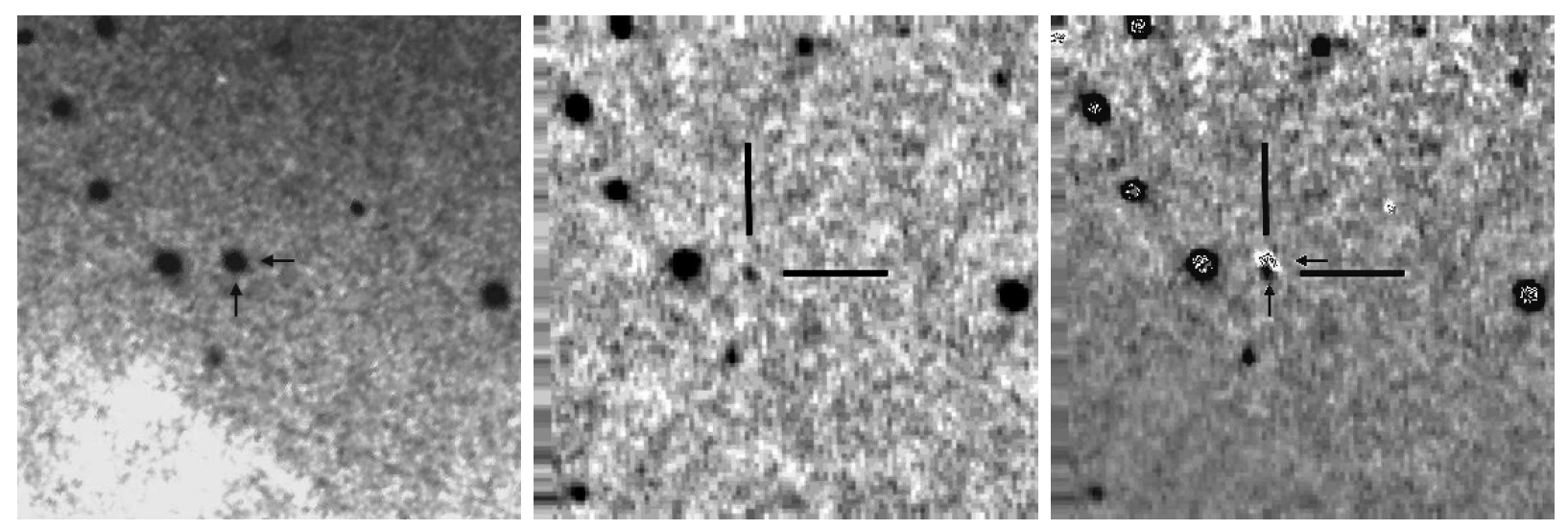

Fig. 29.- Images of M31N 1955-09b, M31N 2012-03b and their comparison (left, center, and right, respectively). As can be seen from the comparison image, M31N 2012-03b is clearly not coincident with 1955-09b (in white), and we conclude that the former nova is not a recurrence of the latter. The image of M31N 1955-09b is from Rosino (1964), while that of 2012-03b is from Hornoch \& Vrastil (2012b). North is up and East to the left, with a scale of $\sim 2^{\prime}$ on a side. 

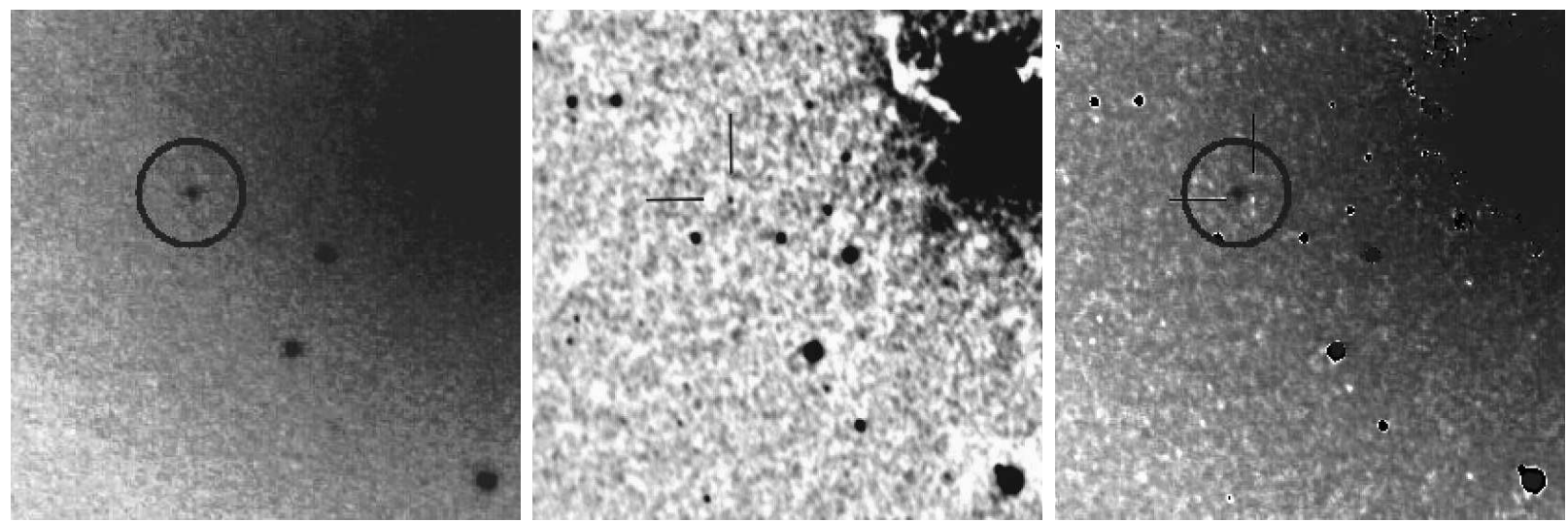

Fig. 30.- Images of M31N 1967-11a, 2006-02a, and their comparison (left, center, and right, respectively). A careful comparison of the images, 1967-11a from Henze et al. (2008a) and 2006-02a from Hornoch (unpublished), shows that the novae are not coincident, with M31N 2006-02a (in white) being 4" WSW of M31N 1967-11a. North is up and East to the left, with a scale of $\sim 2^{\prime}$ on a side.
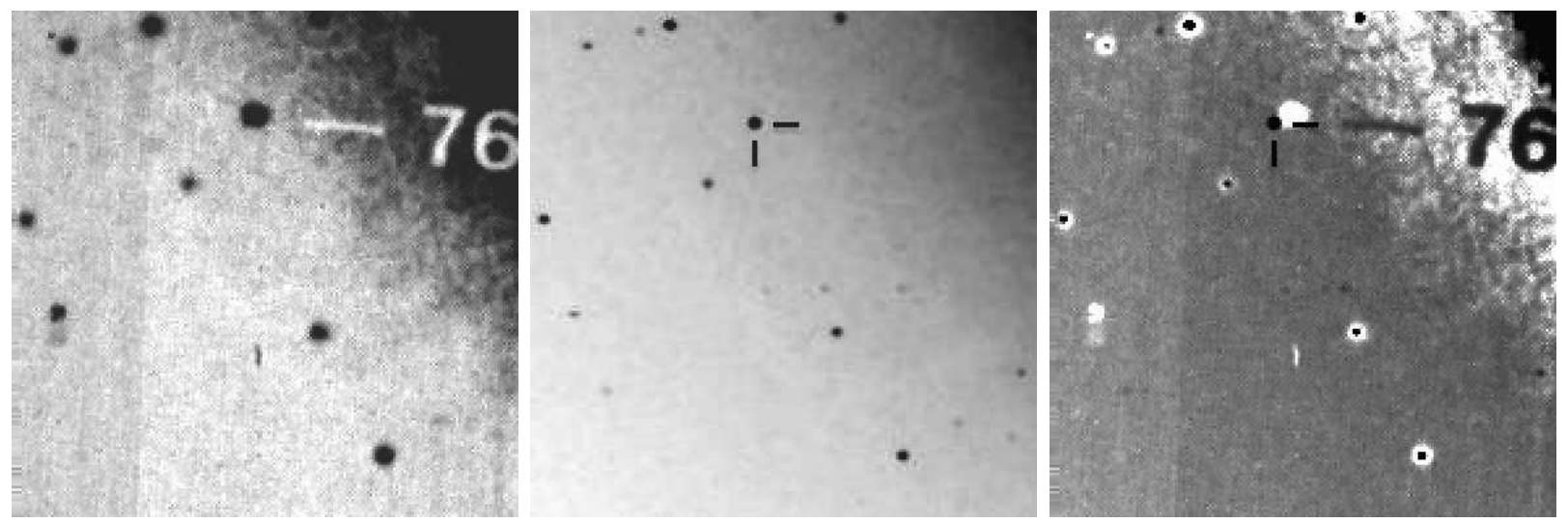

Fig. 31.- Images of M31N 1967-12b, 1999-06b, and their comparison (left, center, and right, respectively). These novae are clearly not coincident, with M31N 1999-06b (black) being located $\sim 6^{\prime \prime}$ ESE of the position of 1967-12b. The chart for 1967-12b is taken from Rosino (1973) and that for 1999-06b from the RBSE project on M31 novae (Rector et al. 1999). North is up and East to the left, with a scale of $\sim 2.2^{\prime}$ on a side. 

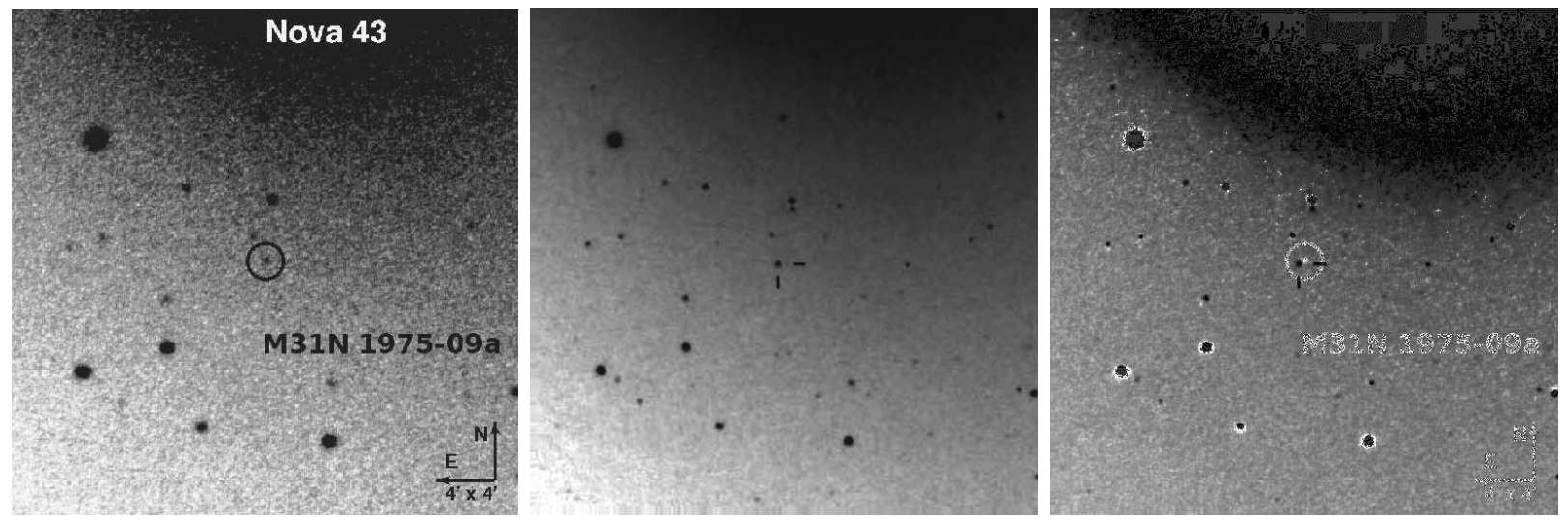

Fig. 32.- Images of M31N 1975-09a, M31N 1999-01a, and their comparison (left, center, and right, respectively). As revealed by the comparison image, although close, the novae are not spatially coincident with M31N 1999-01a (in white) being located $\sim 3.3^{\prime \prime}$ SE of the position of 1975-09a. The chart for 1975-09a is from Henze et al. (2008a), with that for 1999-01a taken from the RBSE project Rector et al. (1999). North is up and East to the left, with a scale of $\sim 4^{\prime}$ on a side.
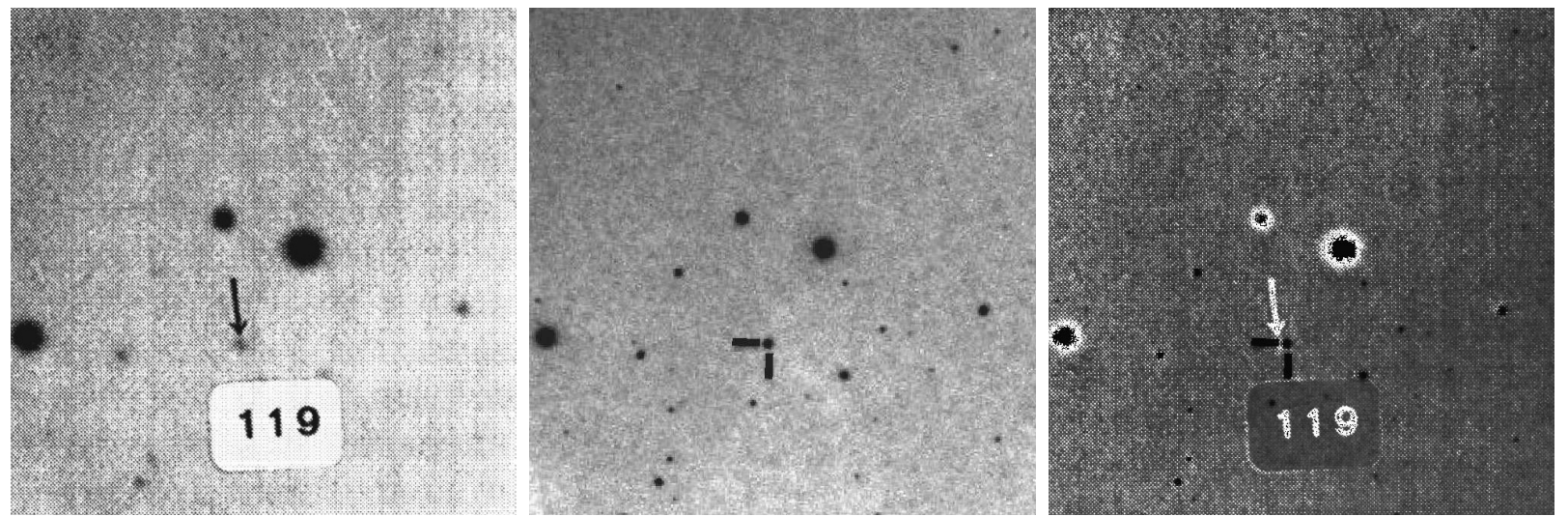

Fig. 33.- Images of M31N 1977-12a, 1998-08a and their comparison (left, center, and right, respectively). A careful inspection of the comparison image reveals that the novae are not coincident, with M31N 1977-12a (in white) being 2.9" ESE of the position of 1998-08a. The chart for M31N 1977-12a is from Rosino et al. (1989), with that for 1998-08a from the RBSE at KPNO (Rector et al. 1999). North is up and East to the left, with a scale of $\sim 2.8^{\prime}$ on a side. 

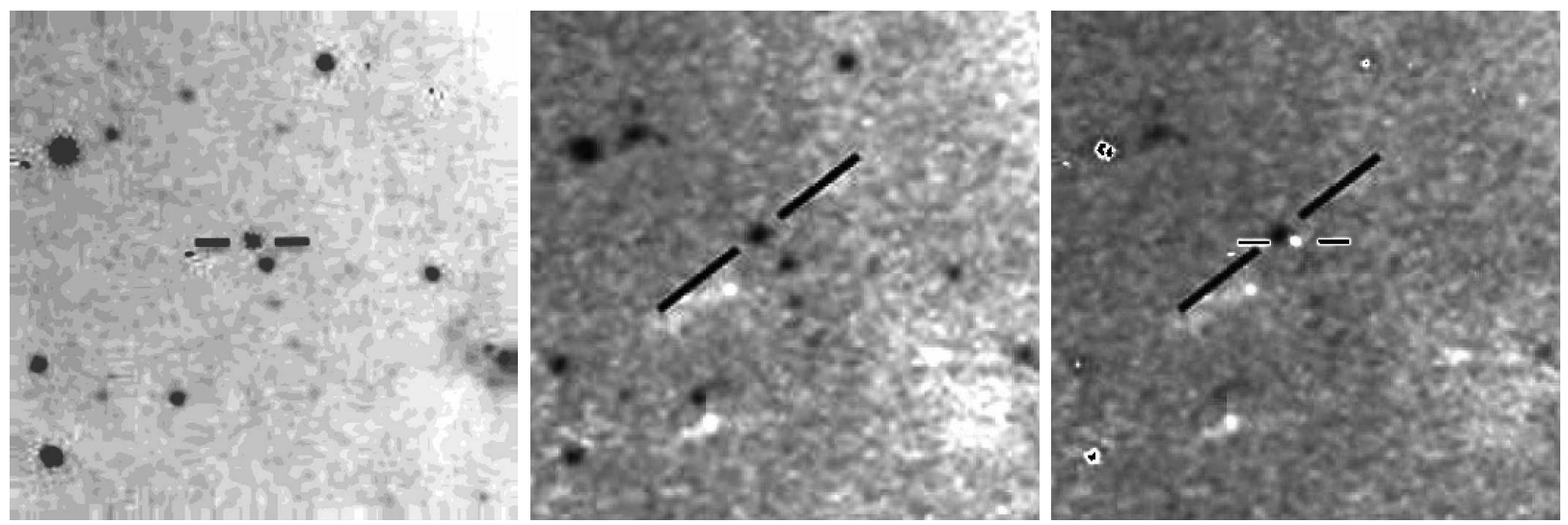

Fig. 34.- Images of M31N 1992-12b, M31N 2001-10f and their comparison (left, center, and right, respectively). As demonstrated by the comparison image, the novae are clearly not coincident, with M31N 2001-10f (black) being 5.3" ENE of the position of 1992-12b. The chart for 1992-12b is from the survey of Shafter \& Irby (2001), and that of 2001-10f from Alksnis et al. (2008). North is up and East to the left, with a scale of $\sim 2.5^{\prime}$ on a side.
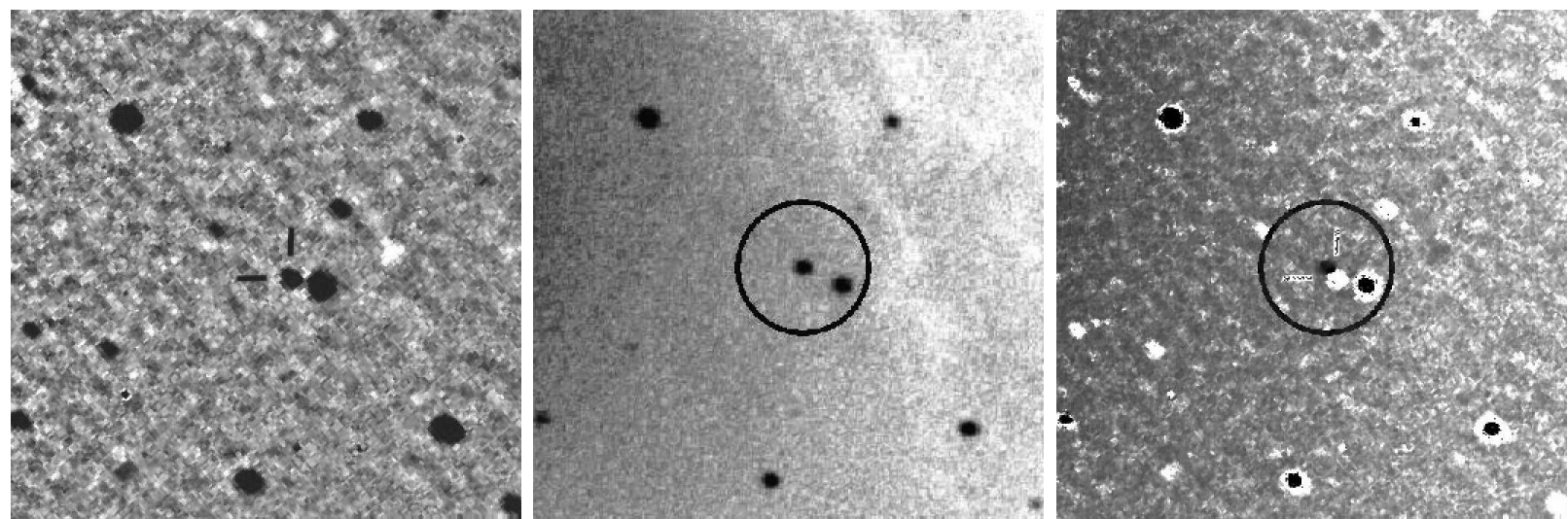

Fig. 35.- Images of M31N 1993-09b, M31N 1996-08g, and their comparison (left, center, and right, respectively). The novae are clearly not coincident, with M31N 1996-08g (in black) being $\sim 5.5^{\prime \prime} \mathrm{NE}$ of the position of 1993-09b. The chart for 1993-09b is taken from the survey of Shafter \& Irby (2001), with that for 1996-08g from Henze et al. (2008a). North is up and East to the left, with a scale of $\sim 2.6^{\prime}$ on a side. 

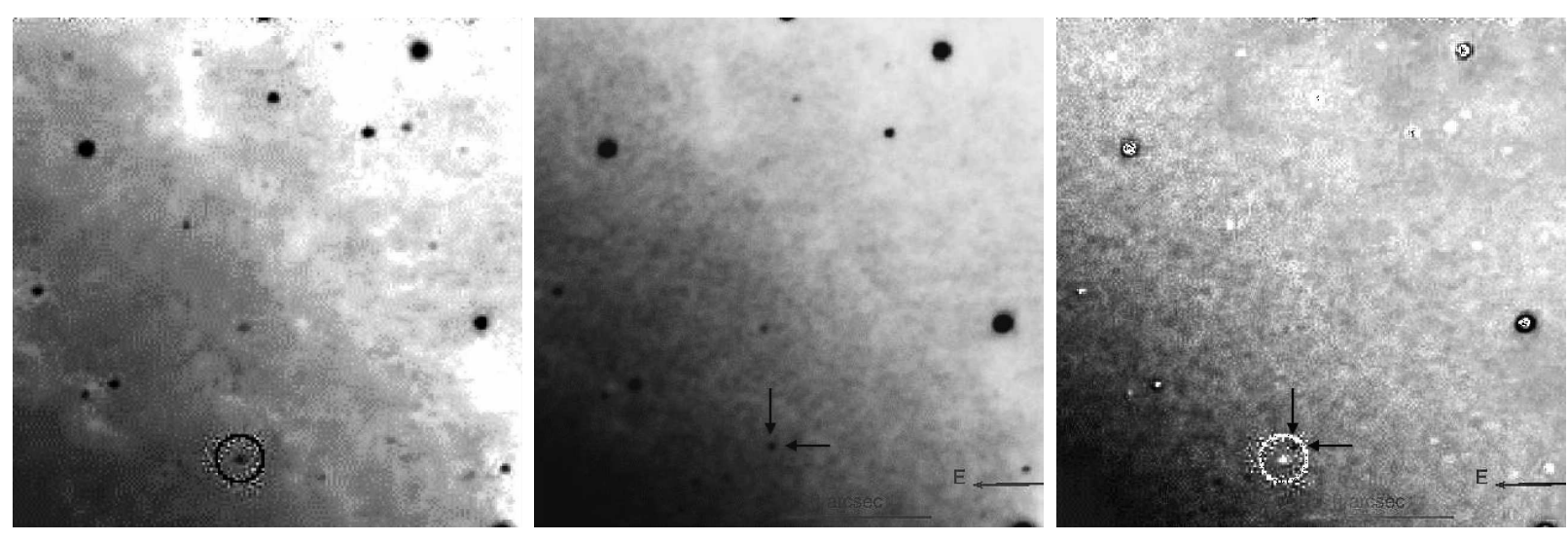

Fig. 36. - Images of M31N 2001-08d Massey et al. (2006), M31N 2008-07a Henze et al. (2008c), and their comparison (left, center, and right, respectively). A careful comparison shows that the novae are not coincident, with M31N 2008-07a (in black) being $~ 3.5^{\prime \prime} \mathrm{NW}$ of the position of 2001-08d. North is up and East to the left, with a scale of $\sim 1.6^{\prime}$ on a side. 

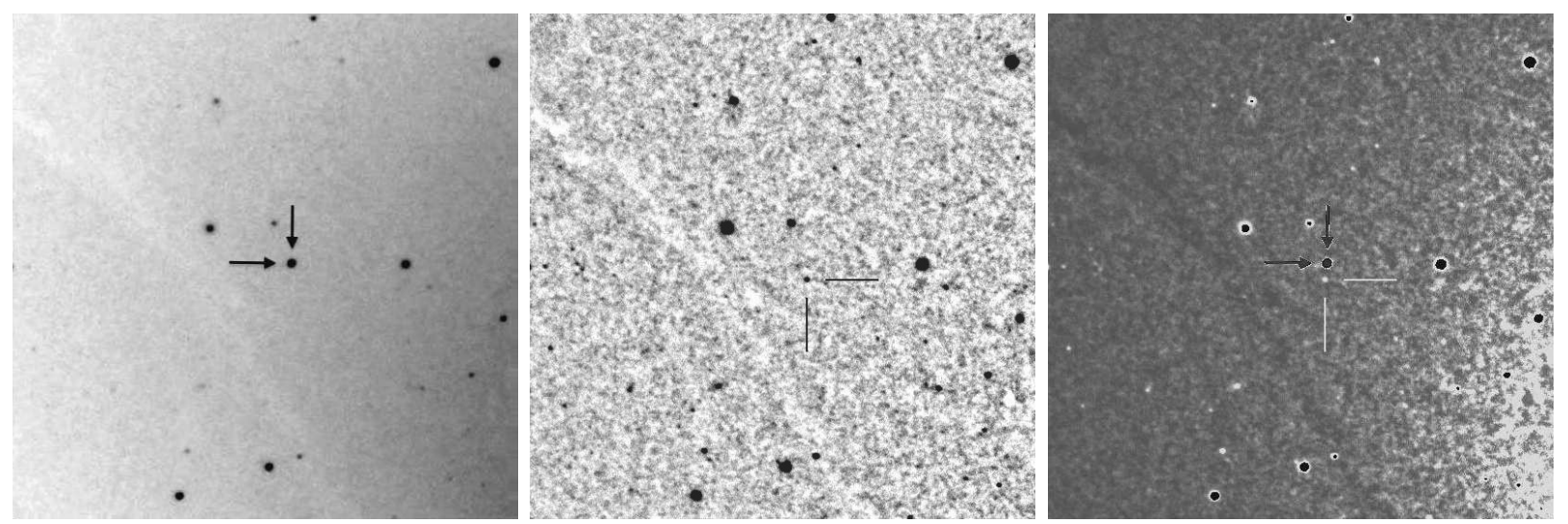

Fig. 37.- Images of M31N 2004-11b, M31N 2010-07b, and their comparison (left, center, and right, respectively). As shown by the comparison image, the novae are clearly not coincident, with M31N 2010-07b (in white) being $~ 5.9^{\prime \prime} \mathrm{S}$ of the position of 2004-11b. The images for 2004-11b and 2010-07b were taken by one of us (K.H.) as part of an ongoing patrol of M31 (Pietsch et al. 2007a; Hornoch et al. 2010c). North is up and East to the left, with a scale of $\sim 3^{\prime}$ on a side.
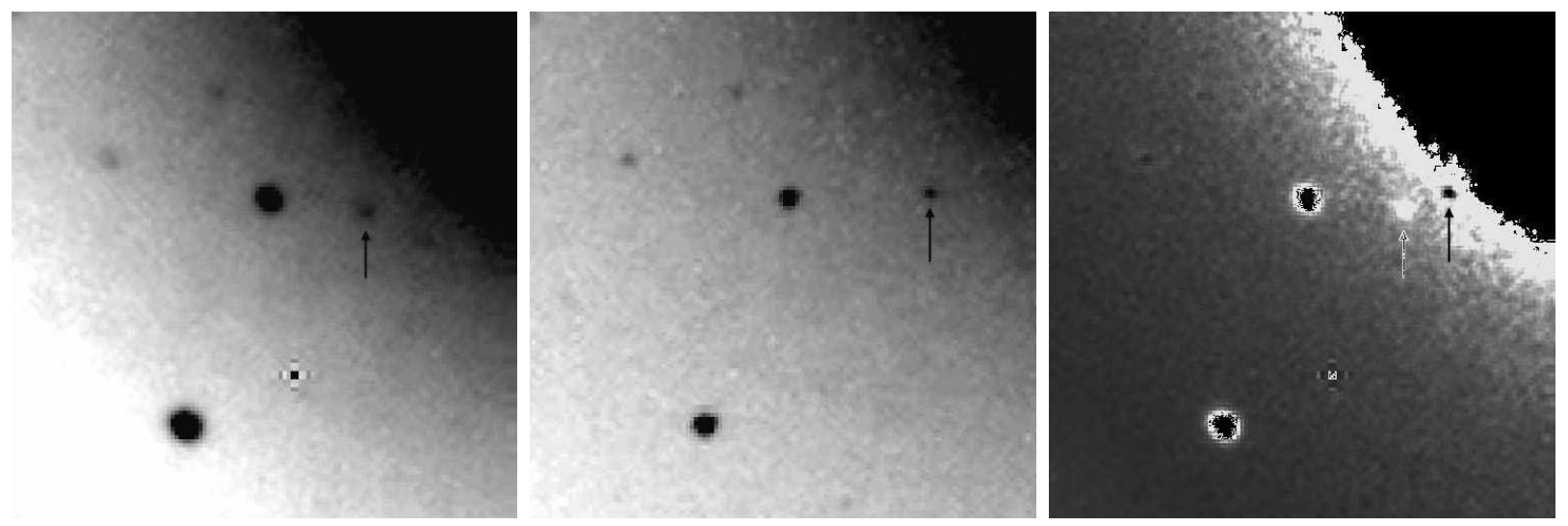

Fig. 38.- Images of M31N 2005-05b, M31N 2009-08d, and their comparison (left, center, and right, respectively). These novae are clearly not coincident, with M31N 2009-08d (in white) being $\sim 5^{\prime \prime}$ WNW of the position of 2005-05b. Images for both novae come from the RBSE M31 nova patrol. North is up and East to the left, with a scale of $\sim 0.8^{\prime}$ on a side. 

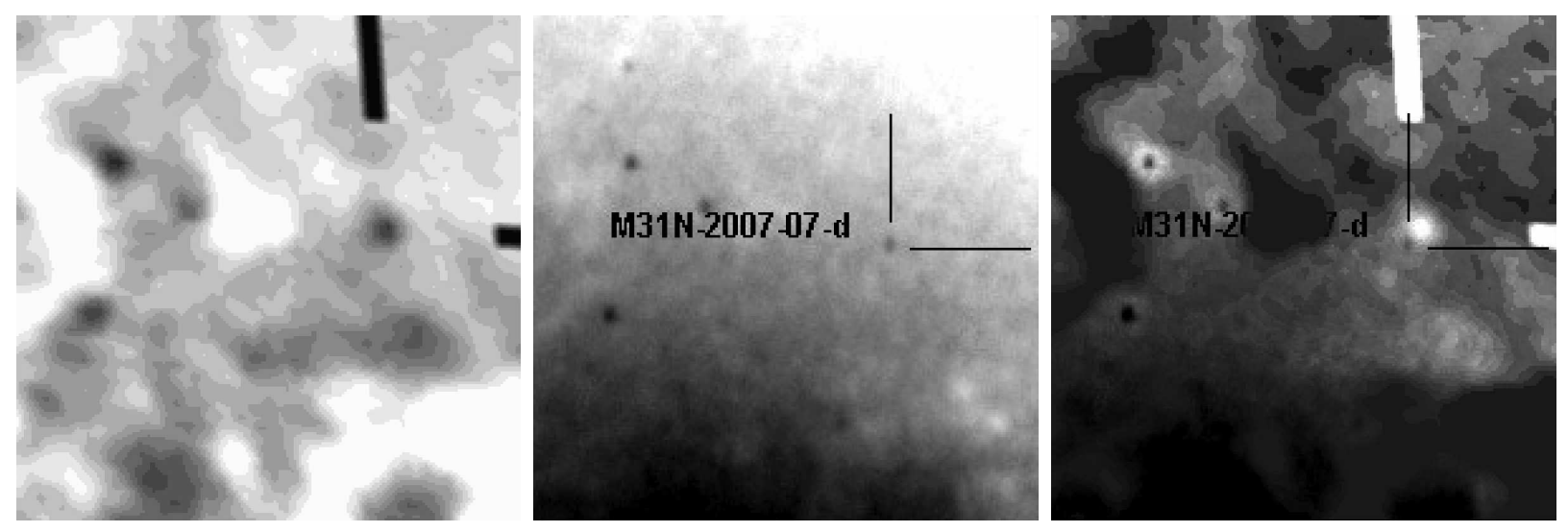

Fig. 39.- Images of M31N 2006-12c, M31N 2007-07e, and their comparison (left, center, and right, respectively). The novae are clearly not coincident, with M31N 2007-07e (in black) being located $\sim 4.3^{\prime \prime} \mathrm{SE}$ of the position of 2006-12c. The image for M31N 2006-12c was taken from the nova patrol of K.H., while that of M31N 2007-07e is from the survey of Hatzidimitriou et al. (2007b) (Note that the chart for 2007-07e is incorrectly labeled as 2007-07d). North is up and East to the left, with a scale of $\sim 1^{\prime}$ on a side.
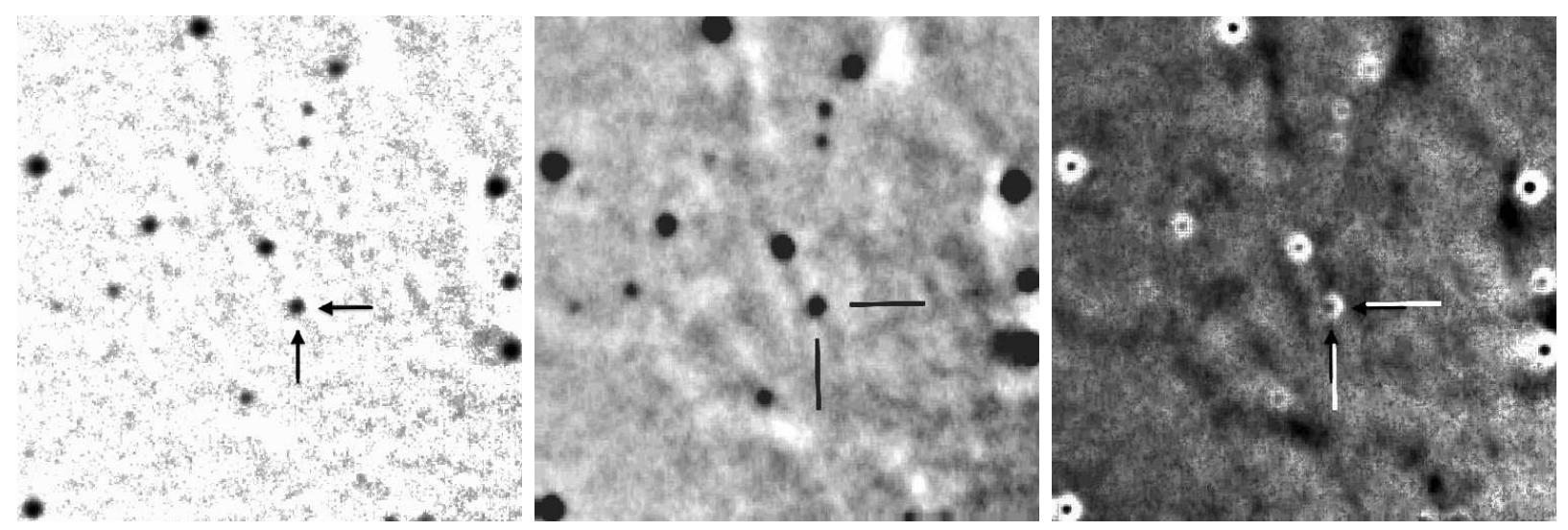

Fig. 40.- Images of M31N 2010-01a, M31N 2010-12c, and their comparison (left, center, and right, respectively). As can be seen from the comparison image, the positions of these novae differ very slightly with M31N 2010-12c (in white) lying just $\sim 0.7^{\prime \prime} \mathrm{NW}$ of the position of M31N 2010-01a. This result is in agreement with the findings of Hornoch et al. (2010a). The image for 2010-01a is from the SuperLOTIS project (Burwitz et al. 2010), while that of 2010-12c is from the nova patrol of K.H. North is up and East to the left, with a scale of $\sim 2.5^{\prime}$ on a side. 


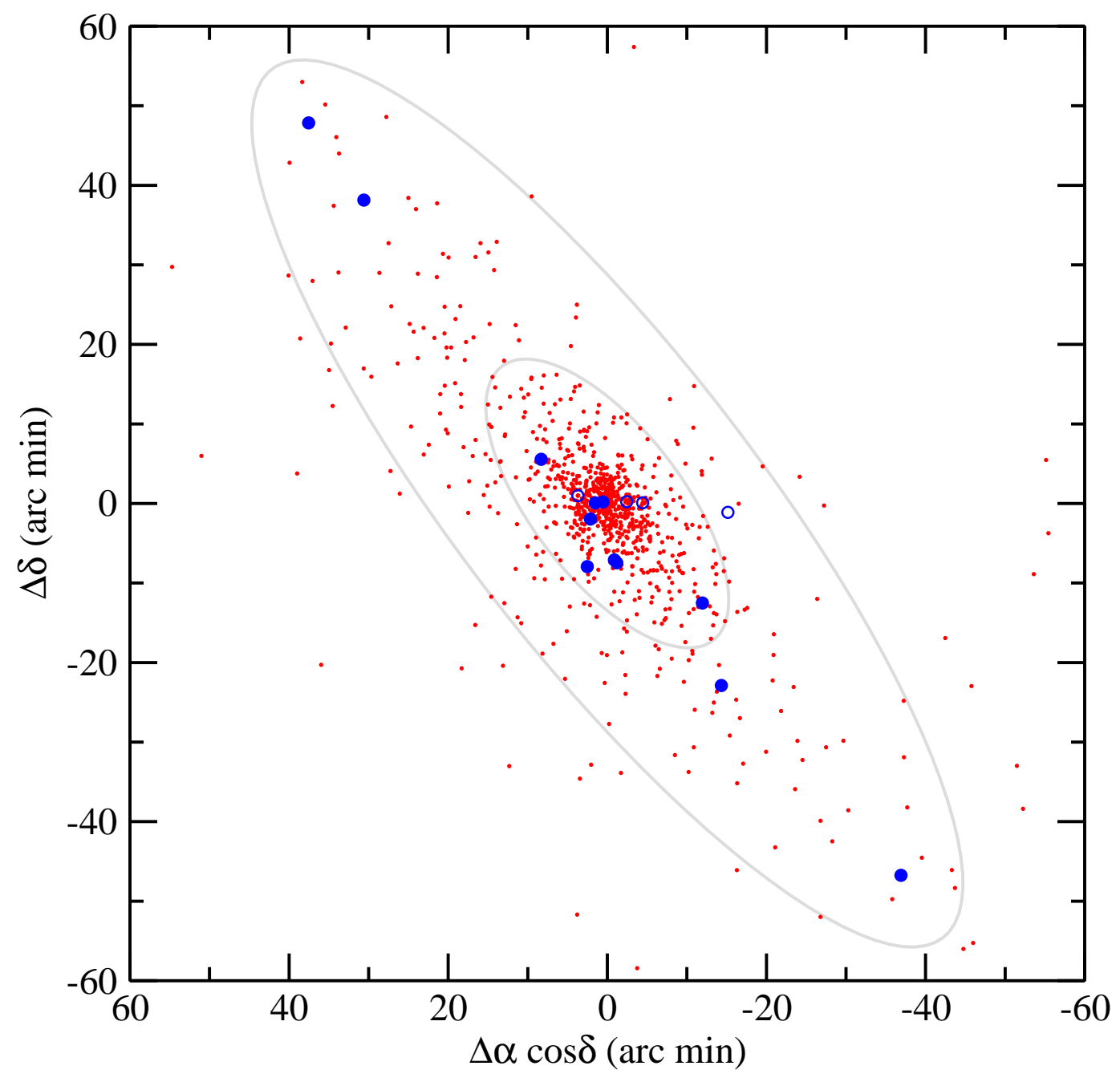

Fig. 41. - The spatial distribution of RNe compared with all novae in M31 (small red points). The filled blue circles represent the confirmed RNe, with the blue open circles showing the positions of the possible RNe. Two $B$-band isophotes are shown to illustrate the orientation of M31 on the sky. 


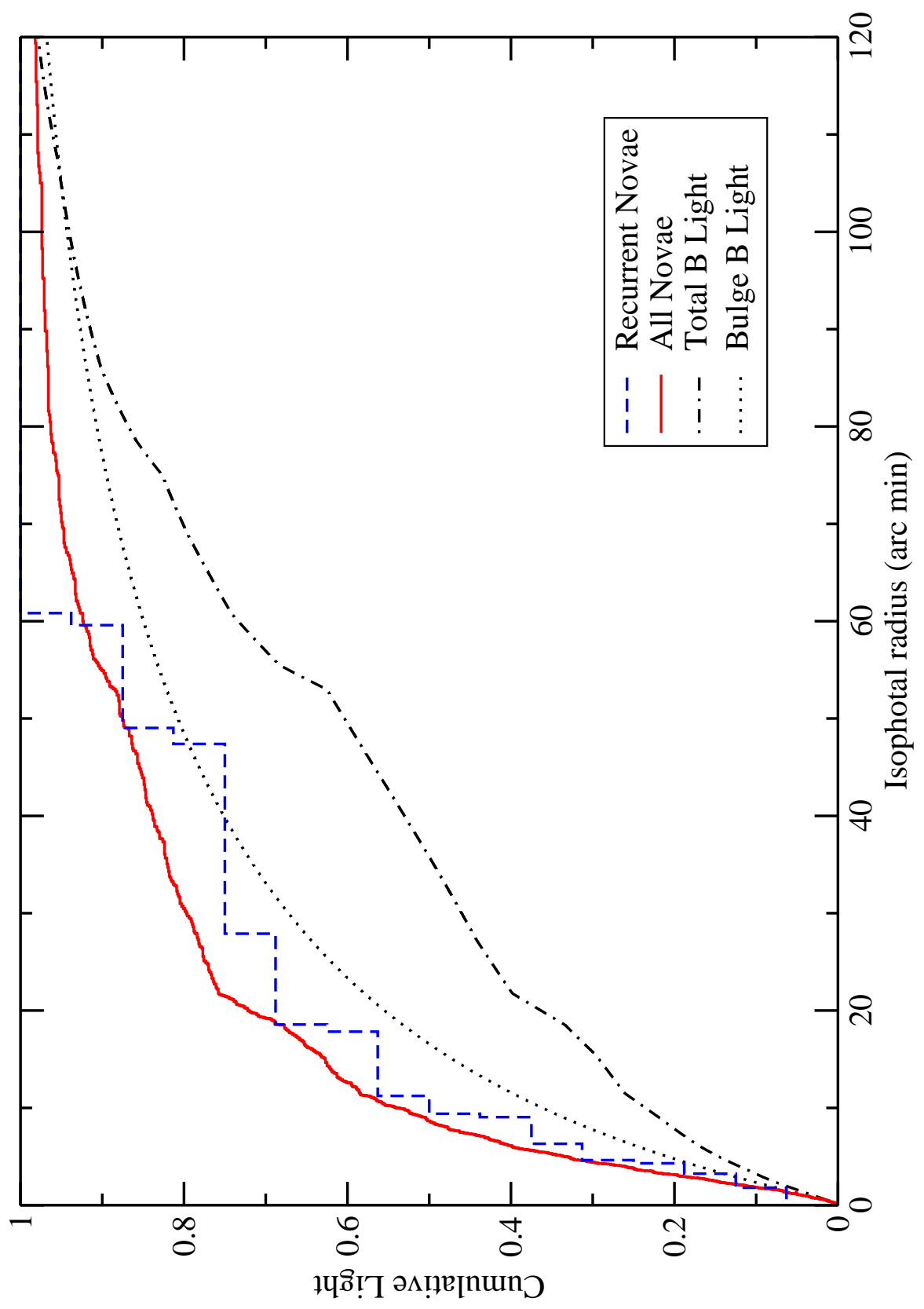

Fig. 42.- The cumulative $\mathrm{RN}$ distribution (including the 4 possible $\mathrm{RNe}$ ) compared with the cumulative distribution for all novae and with the background $B$-band bulge and total light. There is no significant difference between the cumulative distributions for RNe and novae in general $(\mathrm{KS}=0.95)$. The nova distributions fall off faster then the background light, as expected given that the nova surveys are not spatially complete, particularly in the outer regions of M31. 


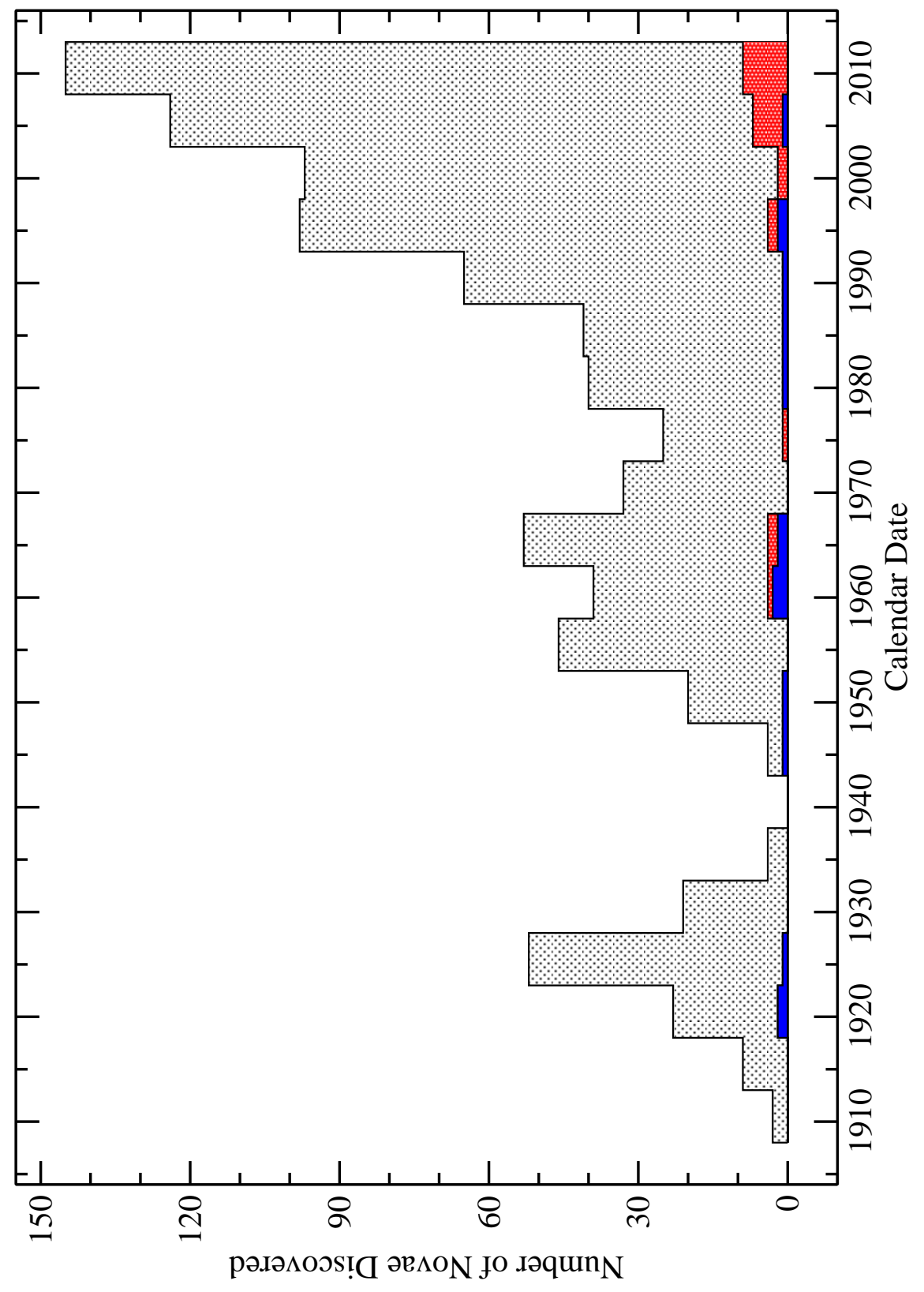

Fig. 43. - The history of nova discoveries in M31. The discovery dates of nova candidates in M31 have been divided into 5 year bins and plotted as a function of time (lightly shaded grey region). The shaded red area shows the overall number of RN outbursts observed in a given time interval, while the dark blue region shows the number of outbursts for newly discovered RNe as a function of time. 


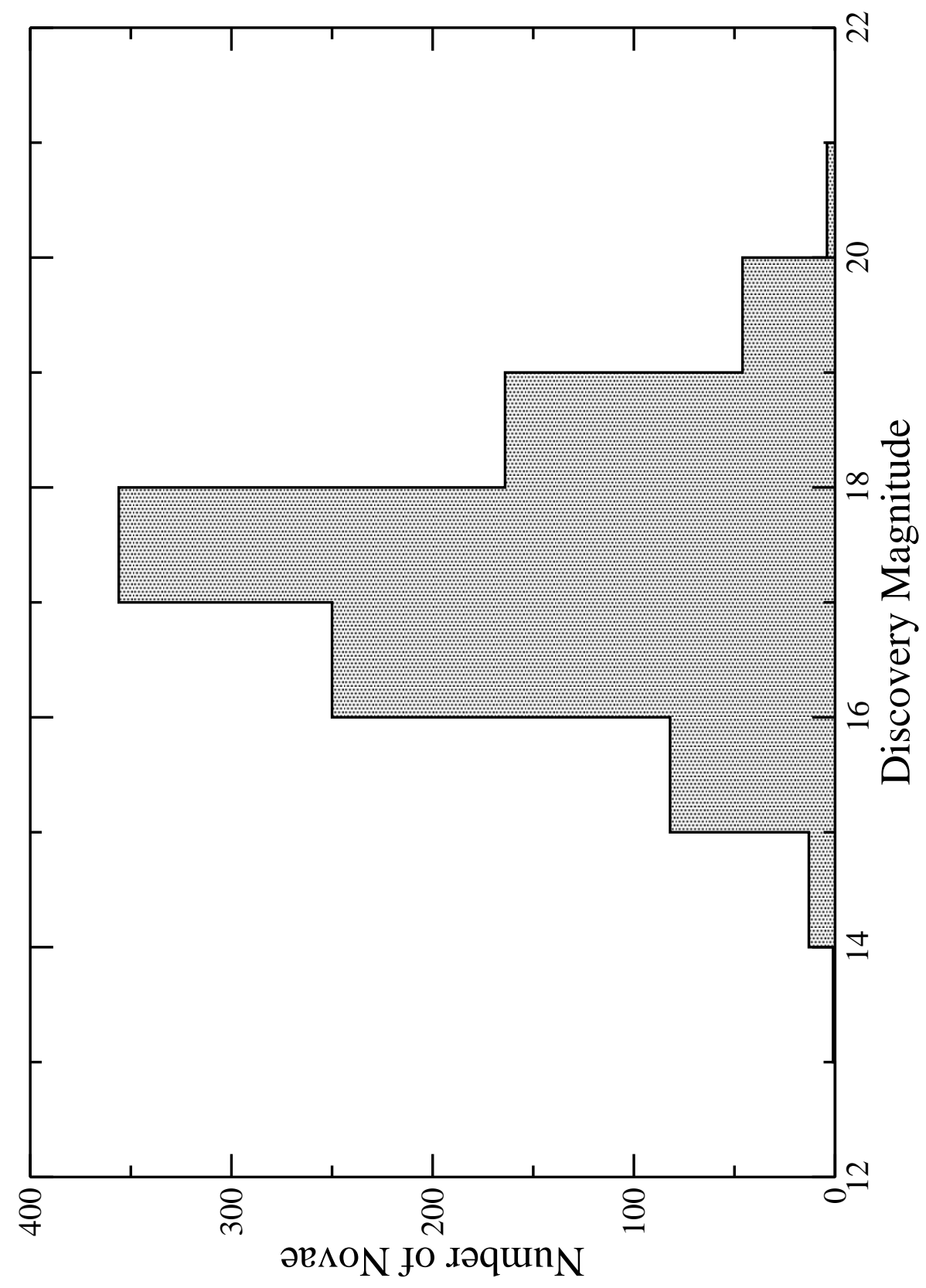

Fig. 44. - The distribution of discovery magnitudes from the online catalog of Pietsch et al. (2007a). The number of novae discovered starts to drop off precipitously for $m \gtrsim 18$. 


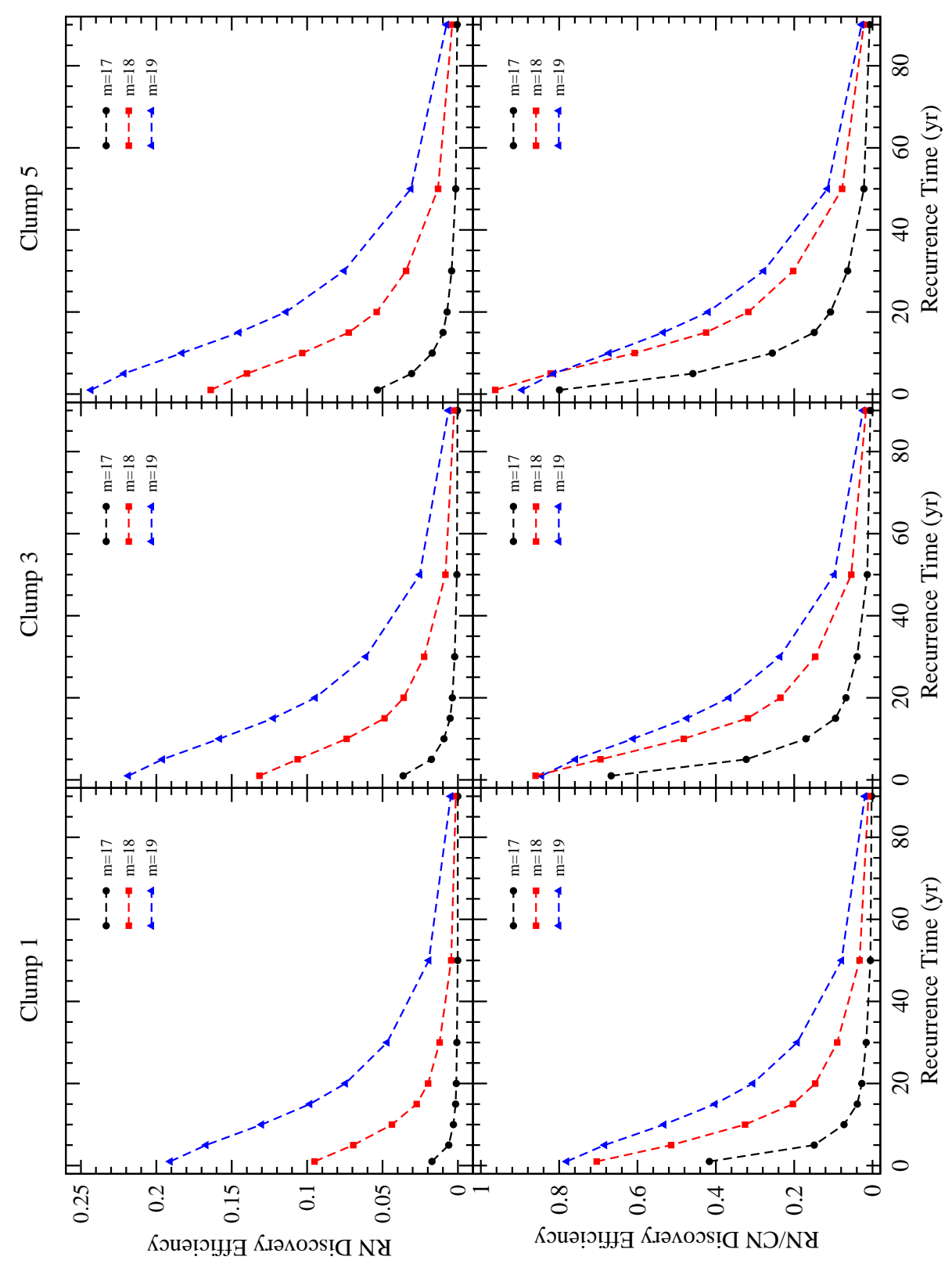

Fig. 45.- Discovery efficiencies for RNe, and for RNe relative to CNe, are plotted as a function of RN recurrence time for the three different assumed limiting magnitudes and temporal coverages $\left(m_{\lim }=17\right.$, black circles; $m_{\text {lim }}=18$, red squares; $m_{\text {lim }}=19$, blue triangles $)$. As expected, the discovery efficiency for RNe is strongly dependent on the recurrence time and on the limiting magnitude of the surveys. 


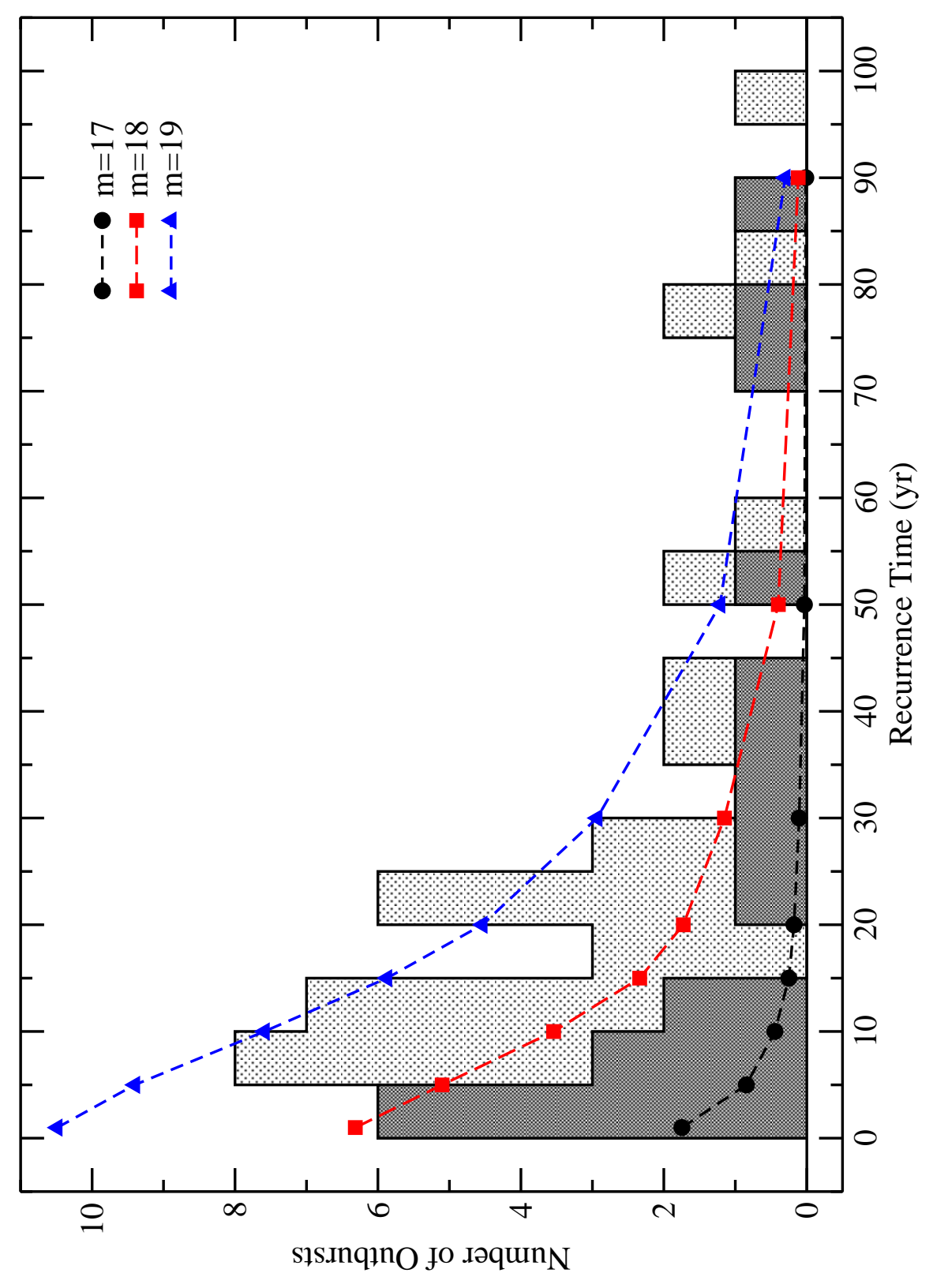

Fig. 46. - The observed RN recurrence time distribution for known RNe in the Galaxy (light shaded region) and M31 (dark shaded region) compared with our Clump 3 model prediction for how the RN discovery efficiencies should vary with recurrence time. 


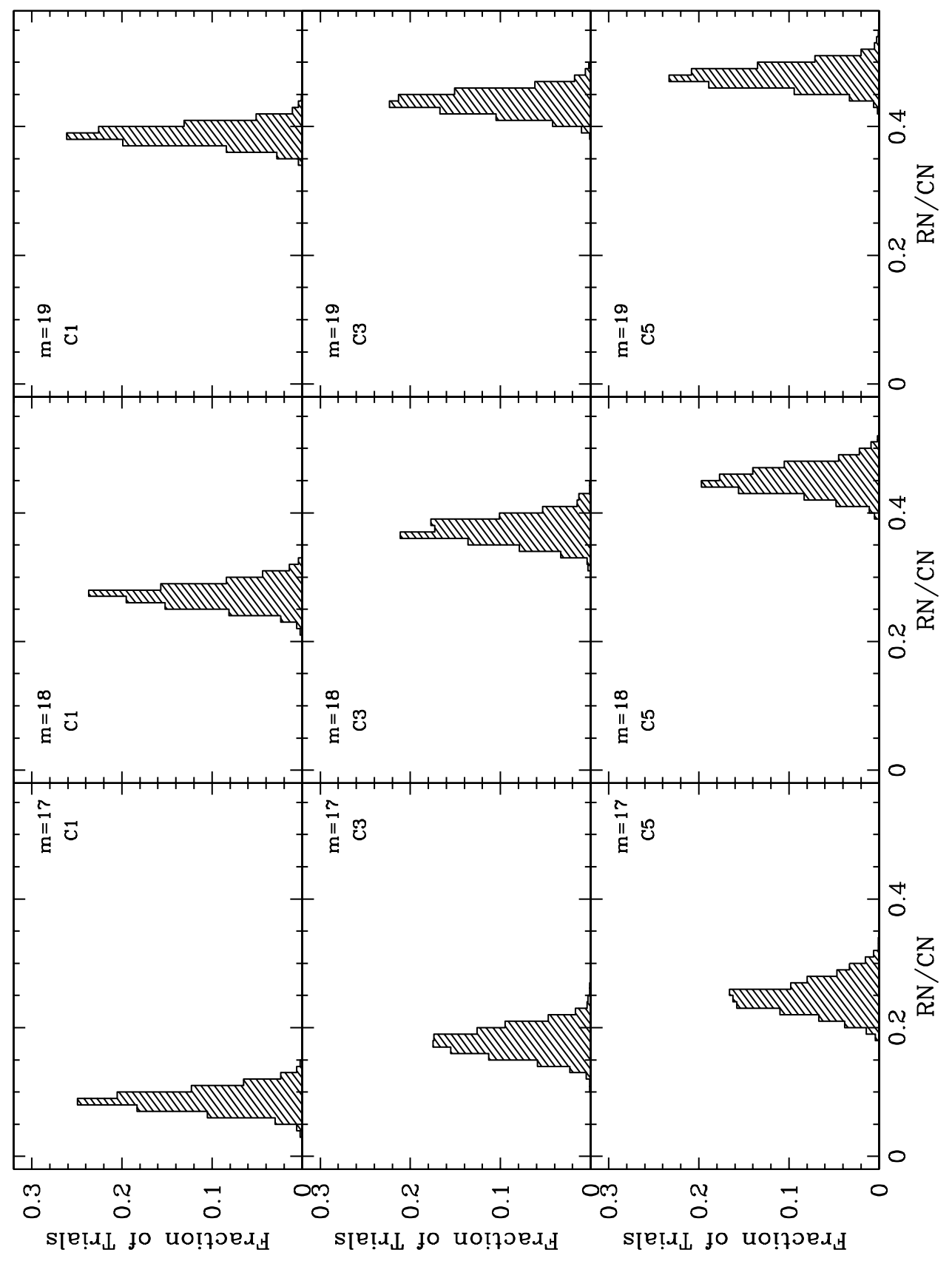

Fig. 47.- The ratio of $\mathrm{RN}$ to $\mathrm{CN}$ outbursts detected in our Monte Carlo simulation for $\mathrm{RN}$ recurrence times chosen at random from a range of 1 to 100 years. Results are shown for assumed limiting magnitudes $m=17, m=18$ and $m=19$ (columns left to right), and temporal coverages Clump 1, Clump 3, and Clump 5 (rows top to bottom). 


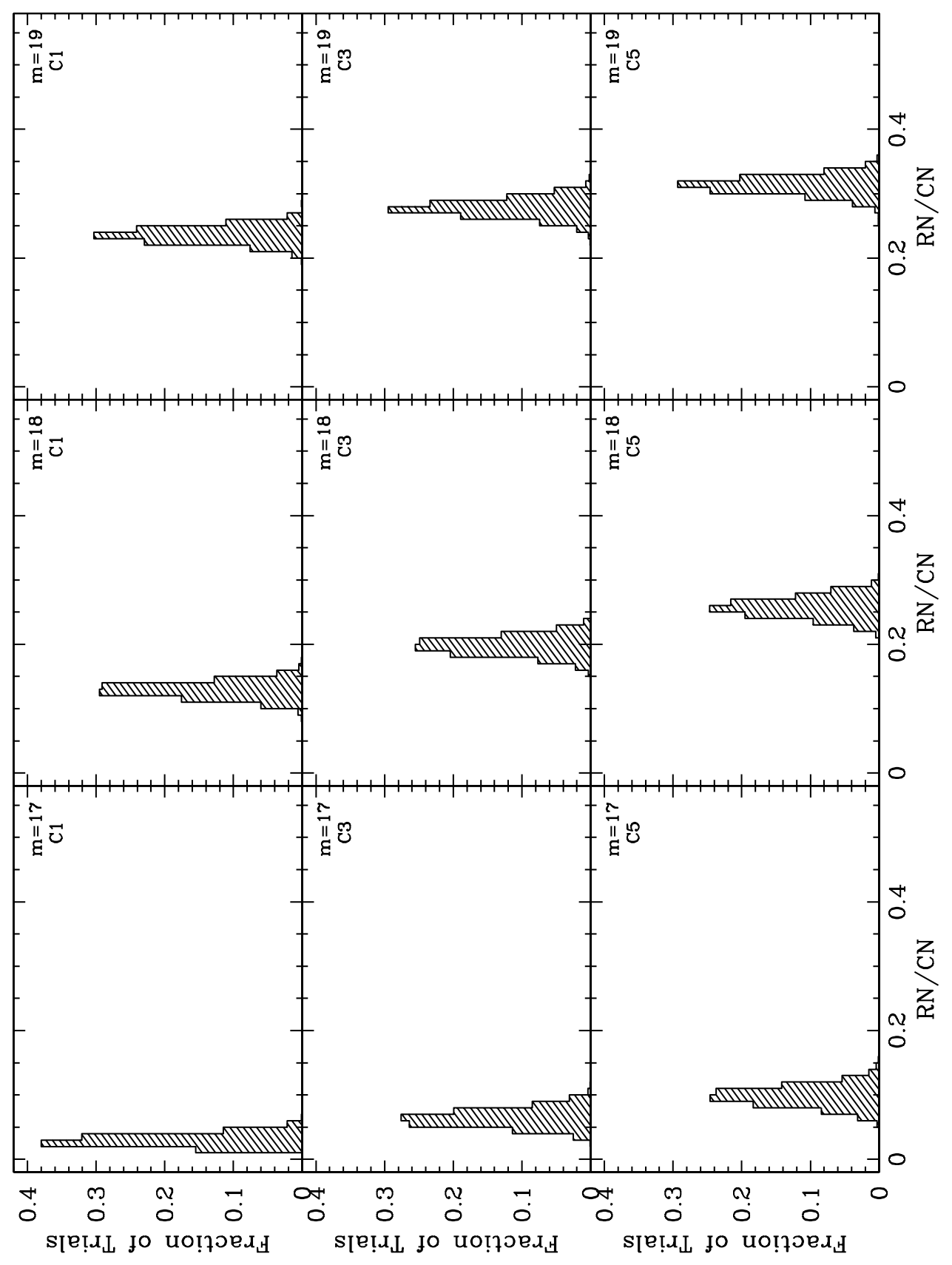

Fig. 48. - Same as Figure 47, except for RN recurrence times chosen randomly from a range of 5 to 100 years. 
Table 1. M31 Recurrent Nova Candidates

\begin{tabular}{|c|c|c|c|c|c|c|c|c|c|}
\hline Nova & Possible Recurrence & $\Delta t(\mathrm{yr})$ & $s\left({ }^{\prime \prime}\right)$ & $a\left({ }^{\prime}\right)$ & $N$ & $P_{C}$ & Type $^{\mathrm{a}}$ & Charts? & Comments \\
\hline M31N 1909-09b & M31N 2009-02b & 99.4 & 4.20 & 3.96 & 64 & 0.8552 & & $\mathrm{Y}$ & Not RN \\
\hline M31N 1918-02b & M31N 2013-10g & 95.7 & 12.18 & 9.54 & 17 & 0.4326 & Fe II & $\mathrm{Y}$ & Not RN \\
\hline M31N 1919-09a & M31N 1998-06a & 78.7 & 1.80 & 10.50 & 27 & 0.0296 & & $\mathrm{Y}$ & $\mathrm{RN}$ \\
\hline M31N 1923-02a & M31N 1967-12a & 44.8 & 6.55 & 1.74 & 69 & 0.9999 & & $\mathrm{Y}$ & Not RN \\
\hline$\ldots$ & M31N 1993-06a & 70.3 & 6.70 & 1.64 & 63 & 0.9999 & & $\mathrm{~N}$ & Not $\mathrm{RN}^{\mathrm{b}}$ \\
\hline$\ldots$ & M31N 1993-11c & 70.7 & 6.48 & 1.73 & 68 & 0.9999 & & $\mathrm{Y}$ & Not RN \\
\hline$\ldots$ & M31N 2013-08b & 90.5 & 6.64 & 1.73 & 68 & 0.9999 & & $\mathrm{Y}$ & Not RN \\
\hline M31N 1967-12a & M31N 1993-11c & 25.9 & 3.30 & 1.73 & 68 & 0.9326 & & $\mathrm{Y}$ & Not RN \\
\hline$\ldots$ & M31N 2013-08b & 45.6 & 1.41 & 1.73 & 68 & 0.3813 & & $\mathrm{Y}$ & Not RN \\
\hline M31N 1993-11c & M31N 2013-08b & 19.9 & 4.66 & 1.73 & 68 & 0.9960 & & $\mathrm{Y}$ & Not RN \\
\hline M31N 1923-12c & M31N 2012-01b & 88.1 & 0.83 & 10.99 & 36 & 0.0112 & $\mathrm{He} / \mathrm{N}$ & $\mathrm{Y}$ & $\mathrm{RN}$ \\
\hline M31N 1924-02b & M31N 1995-09d & 71.6 & 5.20 & 13.57 & 14 & 0.0512 & & $\mathrm{~N}$ & Not Nova \\
\hline M31N 1924-08a & M31N 1987-12a & 63.3 & 3.10 & 2.61 & 81 & 0.9008 & & $\mathrm{Y}$ & Not RN \\
\hline M31N 1925-07c & M31N 2011-12b & 86.4 & 7.55 & 20.30 & 19 & 0.1477 & Fe II & $\mathrm{Y}$ & Not RN \\
\hline M31N 1925-09a & M31N 1976-12a & 51.3 & 9.70 & 19.57 & 15 & 0.1548 & & $\mathrm{Y}$ & Not RN \\
\hline M31N 1926-06a & M31N 1962-11a & 36.5 & 6.27 & 17.45 & 24 & 0.1870 & & $\mathrm{Y}$ & $\mathrm{RN}$ \\
\hline M31N 1926-07c & M31N 1980-09d & 54.2 & 6.95 & 2.01 & 72 & 0.9999 & & $\mathrm{Y}$ & Not $\mathrm{RN}^{\mathrm{c}}$ \\
\hline M31N 1927-08a & M31N 2009-11e & 82.2 & 8.02 & 3.86 & 62 & 0.9990 & & $\mathrm{Y}$ & Not RN \\
\hline M31N 1930-06b & M31N 2008-08e & 78.2 & 6.29 & 12.95 & 29 & 0.3035 & & $\mathrm{~N}$ & Not Nova \\
\hline M31N 1930-06c & M31N 1996-08a & 66.1 & 7.23 & 2.67 & 82 & 0.9999 & & $\mathrm{Y}$ & Not RN \\
\hline M31N 1932-09d & M31N 2001-07d & 68.9 & 3.63 & 3.03 & 80 & 0.9390 & & $\mathrm{~N}$ & Unlikely RN \\
\hline M31N 1945-09c & M31N 1975-11a & 27: & 0.88 & 29.19 & 9 & 0.0004 & & $\mathrm{Y}$ & $\mathrm{RN}$ \\
\hline M31N 1953-09b & M31N 2004-08a & 50.8 & 4.80 & 5.77 & 48 & 0.6399 & & $\mathrm{Y}$ & RN: \\
\hline M31N 1953-11a & M31N 1960-12a & 7.1 & 7.85 & 4.40 & 70 & 0.9996 & & $\mathrm{Y}$ & Not RN \\
\hline$\ldots$ & M31N 1962-11b & 9.1 & 2.90 & 4.38 & 69 & 0.6215 & & $\mathrm{Y}$ & Not RN \\
\hline$\ldots$ & M31N 2013-05b & 59.6 & 6.67 & 4.40 & 70 & 0.9959 & & $\mathrm{Y}$ & Not RN \\
\hline M31N 1960-12a & M31N 1962-11b & 1.9 & 5.31 & 4.38 & 69 & 0.9645 & & $\mathrm{Y}$ & Not RN \\
\hline$\ldots$ & M31N 2013-05b & 52.4 & 1.20 & 4.40 & 70 & 0.1551 & & $\mathrm{Y}$ & $\mathrm{RN}$ \\
\hline M31N 1962-11b & M31N 2013-05b & 50.5 & 4.12 & 4.40 & 70 & 0.8684 & & $\mathrm{Y}$ & Not RN \\
\hline M31N 1954-06c & M31N 2010-01b & 55.6 & 8.78 & 12.39 & 28 & 0.4995 & & $\mathrm{Y}$ & Not RN \\
\hline M31N 1955-09b & M31N 2012-03b & 56.5 & 1.94 & 10.46 & 26 & 0.0319 & & $\mathrm{Y}$ & Not RN \\
\hline M31N 1957-10b & M31N 2010-12a & 53.1 & 1.10 & 51.43 & 5 & 0.0001 & & $\mathrm{~N}$ & Unlikely RN \\
\hline M31N 1961-11a & M31N 2005-06c & 43.6 & 2.37 & 3.03 & 80 & 0.6910 & & $\mathrm{Y}$ & $\mathrm{RN}:$ \\
\hline M31N 1963-09c & M31N 1968-09a & 5.0 & 0.89 & 17.60 & 24 & 0.0041 & & $\mathrm{Y}$ & $\mathrm{RN}$ \\
\hline$\ldots$ & M31N 2001-07b & 37.8 & 1.23 & 17.60 & 24 & 0.0079 & & $\mathrm{~N}$ & $\mathrm{RN}$ \\
\hline$\ldots$ & M31N 2010-10e & 47.1 & 1.32 & 17.60 & 24 & 0.0090 & $\mathrm{He} / \mathrm{N}$ & $\mathrm{Y}$ & $\mathrm{RN}$ \\
\hline M31N 1968-09a & M31N 2001-07b & 32.8 & 0.45 & 17.60 & 24 & 0.0011 & & $\mathrm{Y}$ & $\mathrm{RN}$ \\
\hline$\ldots$ & M31N 2010-10e & 42.1 & 0.49 & 17.60 & 24 & 0.0013 & $\mathrm{He} / \mathrm{N}$ & $\mathrm{Y}$ & $\mathrm{RN}$ \\
\hline M31N 2001-07b & M31N 2010-10e & 9.3 & 0.11 & 17.60 & 24 & 0.0001 & $\mathrm{He} / \mathrm{N}$ & $\mathrm{N}$ & $\mathrm{RN}$ \\
\hline M31N 1964-12b & M31N 1998-07bd & 33.6 & 4.51 & 0.71 & 51 & 0.9981 & & $\mathrm{~N}$ & Not RN \\
\hline M31N 1966-08a & M31N 1968-10c & 2.2 & 0.00 & 31.18 & 3 & 0.0000 & & $\mathrm{Y}$ & RN: \\
\hline M31N 1966-09e & M31N 2007-08d & 40.9 & 0.36 & 59.56 & 7 & 0.0000 & Fe II & $\mathrm{Y}$ & $\mathrm{RN}$ \\
\hline M31N 1967-11a & M31N 2006-02a & 38.2 & 4.18 & 1.46 & 62 & 0.9859 & & $\mathrm{Y}$ & Not RN \\
\hline M31N 1967-12b & M31N 1999-06b & 31.5 & 5.98 & 4.43 & 67 & 0.9811 & & $\mathrm{Y}$ & Not RN \\
\hline M31N 1969-08a & M31N 2007-12b & 38.3 & 4.86 & 14.68 & 11 & 0.0264 & $\mathrm{He} / \mathrm{N}$ & $\mathrm{N}$ & Not RN \\
\hline M31N 1975-09a & M31N 1999-01a & 23.3 & 3.34 & 6.51 & 46 & 0.3337 & & $\mathrm{Y}$ & Not RN \\
\hline M31N 1977-12a & M31N 1998-08a & 20.7 & 2.07 & 10.77 & 32 & 0.0536 & & $\mathrm{Y}$ & Not RN \\
\hline M31N 1982-08b & $\mathrm{M} 31 \mathrm{~N} 1996-08 \mathrm{c}^{\mathrm{e}}$ & 14.0 & 2.99 & 60.77 & 7 & 0.0014 & & $\mathrm{Y}$ & $\mathrm{RN}$ \\
\hline M31N 1982-09a & M31N 2011-02b & 28.5 & 5.46 & 1.08 & 50 & 0.9980 & & $\mathrm{~N}$ & Unlikely RN \\
\hline M31N 1983-09c & M31N 1997-11c & 14.0 & 5.99 & 8.03 & 35 & 0.4932 & & $\mathrm{~N}$ & Unlikely RN \\
\hline
\end{tabular}


Table 1-Continued

\begin{tabular}{|c|c|c|c|c|c|c|c|c|c|}
\hline Nova & Possible Recurrence & $\Delta t(\mathrm{yr})$ & $s\left({ }^{\prime \prime}\right)$ & $a\left({ }^{\prime}\right)$ & $N$ & $P_{C}$ & Type $^{\mathrm{a}}$ & Charts? & Comments \\
\hline M31N 1984-07a & M31N 2001-10c & 17.2 & 1.29 & 0.57 & 40 & 0.2981 & & $\mathrm{~N}$ & Not RN \\
\hline$\ldots$ & M31N 2004-02a & 19.5 & 1.61 & 0.60 & 45 & 0.4896 & & $\mathrm{~N}$ & Not RN \\
\hline$\ldots$ & M31N 2004-11f & 20.3 & 0.49 & 0.57 & 40 & 0.0496 & & $\mathrm{Y}$ & $\mathrm{RN}$ \\
\hline$\ldots$ & M31N 2012-09a & 28.1 & 0.51 & 0.58 & 40 & 0.0520 & $\mathrm{Fe}$ IIb & $\mathrm{Y}$ & $\mathrm{RN}$ \\
\hline M31N 2001-10c & M31N 2004-02a & 2.3 & 2.81 & 0.60 & 45 & 0.8764 & & $\mathrm{~N}$ & Not RN \\
\hline$\ldots$ & M31N 2004-11f & 3.1 & 1.19 & 0.57 & 40 & 0.2588 & & $\mathrm{~N}$ & Not RN \\
\hline$\ldots$ & M31N 2012-09a & 10.9 & 0.80 & 0.58 & 40 & 0.1260 & Fe II & $\mathrm{N}$ & Not RN \\
\hline M31N 2004-02a & M31N 2004-11f & 0.7 & 2.02 & 0.57 & 40 & 0.5827 & & $\mathrm{~N}$ & Not RN \\
\hline$\ldots$ & M31N 2012-09a & 8.6 & 2.10 & 0.58 & 40 & 0.6051 & $\mathrm{Fe} I \mathrm{II}$ & $\mathrm{N}$ & Not RN \\
\hline M31N 2004-11f & M31N 2012-09a & 7.9 & 0.45 & 0.58 & 40 & 0.0418 & $\mathrm{Fe} I \mathrm{II}$ & $\mathrm{Y}$ & $\mathrm{RN}$ \\
\hline M31N 1984-09b & M31N 2012-12a & 28.2 & 1.52 & 1.27 & 62 & 0.4498 & $\mathrm{Fe}$ II & $\mathrm{N}$ & Unlikely RN \\
\hline M31N 1985-09d & M31N 2009-08d & 23.9 & 4.12 & 0.81 & 53 & 0.9931 & & $\mathrm{~N}$ & Unlikely RN \\
\hline M31N 1985-10c & M31N 1995-12a & 10.2 & 2.40 & 0.31 & 29 & 0.7077 & & $\mathrm{~N}$ & Unlikely RN \\
\hline$\ldots$ & M31N 2003-10b & 18.0 & 1.60 & 0.30 & 29 & 0.4216 & & $\mathrm{~N}$ & Unlikely RN \\
\hline M31N 1995-12a & M31N 2003-10b & 7.9 & 3.66 & 0.30 & 29 & 0.9529 & & $\mathrm{~N}$ & Unlikely RN \\
\hline M31N 1986-09a & M31N 2006-09b & 20.0 & 4.80 & 1.63 & 61 & 0.9943 & & $\mathrm{~N}$ & Unlikely RN \\
\hline M31N 1990-10a & $M 31 N 1997-10 b^{f}$ & 7.0 & 2.26 & 4.24 & 73 & 0.4877 & & $\mathrm{~N}$ & Not RN \\
\hline$\ldots$ & M31N 2007-07a & 16.7 & 0.80 & 4.28 & $\ldots$ & 0.0726 & & $\mathrm{Y}$ & $\mathrm{RN}$ : \\
\hline M31N 1992-12b & M31N 2001-10f & 9.0 & 5.31 & 12.91 & 31 & 0.2563 & & $\mathrm{Y}$ & Not RN \\
\hline M31N 1993-09b & M31N 1996-08g & 2.9 & 5.47 & 5.33 & 55 & 0.8427 & & $\mathrm{Y}$ & Not RN \\
\hline M31N 1997-10f & M31N 2008-08b & 10.8 & 0.45 & 1.69 & 65 & 0.0452 & $\mathrm{He} / \mathrm{N} ?$ & $\mathrm{Y}$ & $\mathrm{RN}$ \\
\hline M31N 1997-11k & M31N 2001-12b & 4.2 & 0.00 & 10.57 & 30 & 0.0000 & & $\mathrm{Y}$ & $\mathrm{RN}$ \\
\hline$\ldots$ & M31N 2009-11b & 12.1 & 0.38 & 10.56 & $\ldots$ & 0.0016 & $\mathrm{Fe} I \mathrm{II}$ & $\mathrm{Y}$ & $\mathrm{RN}$ \\
\hline M31N 2001-12b & M31N 2009-11b & 7.9 & 0.38 & 10.56 & 30 & 0.0016 & & $\mathrm{Y}$ & $\mathrm{RN}$ \\
\hline M31N 2001-08d & M31N 2008-07a & 6.8 & 3.51 & 4.34 & 70 & 0.7739 & Fe II & $\mathrm{Y}$ & Not RN \\
\hline M31N 2004-11b & M31N 2010-07b & 5.6 & 5.91 & 4.97 & 63 & 0.9539 & & $\mathrm{Y}$ & Not RN \\
\hline M31N 2005-05b & M31N 2009-08d & 4.2 & 4.59 & 0.81 & 53 & 0.9981 & $\mathrm{Fe}$ II & $\mathrm{Y}$ & Not RN \\
\hline M31N 2006-11b & M31N 2006-12d & 0.1 & 0.34 & 1.27 & 62 & 0.0292 & & $\mathrm{Y}$ & Not RN \\
\hline M31N 2006-12c & M31N 2007-07e & 1 & 4.39 & 1.99 & 74 & 0.9928 & Fe II & $\mathrm{Y}$ & Not RN \\
\hline M31N 2008-12a & M31N 2009-12bg & 1.0 & $\ldots$ & 48.92 & $\ldots$ & $\ldots$ & & $\mathrm{Y}$ & $\mathrm{RN}$ \\
\hline$\ldots$ & M31N 2011-10e & 2.8 & 0.30 & 48.92 & 11 & 0.0000 & & $\mathrm{Y}$ & $\mathrm{RN}$ \\
\hline$\ldots$ & M31N 2012-10a & 3.8 & 0.74 & 48.92 & 11 & 0.0003 & $\mathrm{He} / \mathrm{N}$ & $\mathrm{Y}$ & $\mathrm{RN}$ \\
\hline$\ldots$ & M31N 2013-11f & 4.9 & 1.01 & 48.93 & 11 & 0.0005 & & $\mathrm{Y}$ & $\mathrm{RN}$ \\
\hline M31N 2011-10e & M31N 2012-10a & 1.0 & 0.46 & 48.92 & 11 & 0.0001 & $\mathrm{He} / \mathrm{N}$ & $\mathrm{Y}$ & $\mathrm{RN}$ \\
\hline$\ldots$ & M31N 2013-11f & 2.1 & 0.84 & 48.93 & 11 & 0.0004 & & $\mathrm{Y}$ & $\mathrm{RN}$ \\
\hline M31N 2012-10a & M31N 2013-11f & 1.1 & 0.89 & 48.93 & 11 & 0.0004 & & $\mathrm{Y}$ & $\mathrm{RN}$ \\
\hline M31N 2010-01a & M31N 2010-12c & 0.9 & 0.82 & 2.69 & 80 & 0.1410 & Fe II & $\mathrm{Y}$ & Not RN \\
\hline
\end{tabular}

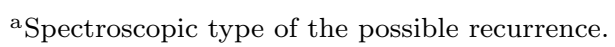

${ }^{\mathrm{b}}$ Although a chart for M31N 1993-06a does not exist, our revised coordinates for 1923-02a differ from those of 1993-06a by $11.6^{\prime \prime}$ making it extremely unlikely that the two novae are coincident.

${ }^{\mathrm{c}}$ Unexpectedly, M31N 1926-07c was found to be coincident with 1997-10f and 2008-08b.

d M31N 1998-07b is not a nova

e The nova was discovered on 1997 August 01; not 1996 August 12 as reported in Shafter \& Irby (2001).

${ }^{\mathrm{f}} \mathrm{M} 31 \mathrm{~N} 1997-10 \mathrm{~b}$ is not a nova.

g We have designated the outburst just announced in Tang et al. (2014) as M31N 2009-12b. 
Table 2. Revised Coordinates ${ }^{\mathrm{a}}$

\begin{tabular}{ccc}
\hline \hline & R.A. & $\begin{array}{c}\text { Decl. } \\
\text { Nova }\end{array}$ \\
\hline M31N 1909-09b & 004227.48 & 411332.4 \\
M31N 1918-02b & 004323.76 & 412134.5 \\
M31N 1919-09a & 004328.65 & 412142.1 \\
M31N 1923-02a & 004250.03 & 411720.9 \\
M31N 1923-12c & 004238.07 & 410841.4 \\
M31N 1924-08a & 004231.49 & 411525.4 \\
M31N 1925-07c & 004355.28 & 412128.4 \\
M31N 1925-09a & 004336.56 & 413156.2 \\
M31N 1926-06a & 004140.66 & 410333.7 \\
M31N 1926-07c & 004252.37 & 411612.8 \\
M31N 1927-08a & 004236.08 & 411306.4 \\
M31N 1930-06c & 004235.26 & 411137.7 \\
M31N 1945-09c & 004128.58 & 405313.7 \\
M31N 1953-09b & 004220.69 & 411607.9 \\
M31N 1953-11a & 004256.10 & 411418.4 \\
M31N 1954-06c & 004259.97 & 412524.2 \\
M31N 1960-12a & 004255.71 & 411412.5 \\
M31N 1961-11a & 004231.36 & 411621.0 \\
M31N 1962-11b & 004255.92 & 411416.6 \\
M31N 1966-09e & 003930.33 & 402914.0 \\
M31N 1977-12a & 004213.70 & 410744.4 \\
M31N 1982-08b & 004606.68 & 420349.3 \\
M31N 1984-07a & 004247.15 & 411619.6 \\
M31N 1987-12a & 004232.91 & 411514.2 \\
M31N 1990-10a & 004304.00 & 411708.1 \\
M31N 1992-12b & 004153.77 & 410721.9 \\
M31N 1993-09b & 004219.53 & 411404.2 \\
M31N 1993-11c & 004250.10 & 411729.4 \\
M31N 1996-08a & 004243.23 & 411814.8 \\
M31N 1996-08c & 004606.60 & 420349.4 \\
M31N 1997-10f & 004252.34 & 411612.8 \\
M31N 1998-08a & 004213.45 & 410744.9 \\
M31N 2010-01a & 004256.68 & 411721.2 \\
M31N 2010-12c & 004256.65 & 411722.3 \\
\hline
\end{tabular}

a Uncertainties estimated to be $0.1 \mathrm{~s}$ in R.A. and $1^{\prime \prime}$ in Decl. 
Table 3. M31 Recurrent Novae

\begin{tabular}{|c|c|c|c|c|c|c|c|}
\hline Nova & Recurrence & $\Delta t(\mathrm{yr})$ & $s\left({ }^{\prime \prime}\right)$ & $a\left({ }^{\prime}\right)$ & $P_{C}$ & Type & Comments \\
\hline M31N 1919-09a & M31N 1998-06a & 78.7 & 1.33 & 10.50 & 0.0163 & & $\mathrm{RN}$ \\
\hline $\mathrm{M} 31 \mathrm{~N} 1923-12 \mathrm{c}$ & M31N 2012-01b & 88.1 & 0.45 & 10.99 & 0.0033 & $\mathrm{He} / \mathrm{N}$ & $\mathrm{RN}$ \\
\hline M31N 1926-06a & M31N 1962-11a & 36.5 & 1.40 & 17.45 & 0.0102 & & $\mathrm{RN}$ \\
\hline \multirow[t]{2}{*}{ M31N 1926-07c } & M31N 1997-10f & 71.3 & 0.34 & 1.68 & 0.0240 & & $\mathrm{RN}$ \\
\hline & M31N 2008-08b & 10.8 & 0.15 & 1.69 & 0.0050 & $\mathrm{He} / \mathrm{N} ?$ & $\mathrm{RN}$ \\
\hline M31N 1945-09c & M31N 1975-11a & $27:$ & 0.41 & 29.19 & 0.0001 & & $\mathrm{RN}$ \\
\hline M31N 1953-09b & M31N 2004-08a & 50.8 & 1.79 & 5.77 & 0.1310 & & $\mathrm{RN}:$ \\
\hline M31N 1960-12a & M31N 2013-05b & 52.4 & 0.90 & 4.40 & 0.0909 & & $\mathrm{RN}$ \\
\hline M31N 1961-11a & M31N 2005-06c & 43.6 & 0.45 & 3.03 & 0.0417 & & $\mathrm{RN}$ : \\
\hline M31N 1963-09c & M31N 1968-09a & 5.0 & 0.89 & 17.61 & 0.0041 & & $\mathrm{RN}$ \\
\hline$\ldots$ & M31N 2001-07b & 32.8 & 1.23 & 17.60 & 0.0079 & & $\mathrm{RN}$ \\
\hline$\ldots$ & M31N 2010-10e & 9.3 & 1.32 & 17.60 & 0.0090 & $\mathrm{He} / \mathrm{N}$ & $\mathrm{RN}$ \\
\hline M31N 1966-08a & M31N 1968-10c & 2.2 & 0.00 & 31.18 & 0.0000 & & $\mathrm{RN}:$ \\
\hline M31N 1966-09e & M31N 2007-08d & 40.9 & 0.71 & 59.56 & 0.0001 & Fe II & RN \\
\hline M31N 1982-08b & $\mathrm{M} 31 \mathrm{~N} 1996-08 \mathrm{c}^{\mathrm{a}}$ & 14.0 & 0.90 & 60.77 & 0.0001 & & $\mathrm{RN}$ \\
\hline M31N 1984-07a & M31N 2004-11f & 20.3 & 0.31 & 0.57 & 0.0197 & & $\mathrm{RN}$ \\
\hline$\ldots$ & M31N 2012-09a & 7.8 & 0.57 & 0.58 & 0.0660 & Fe IIb & $\mathrm{RN}$ \\
\hline M31N 1990-10a & M31N 2007-07a & 16.7 & 0.59 & 4.28 & 0.0411 & & RN: \\
\hline M31N 1997-11k & M31N 2001-12b & 4.2 & 0.00 & 10.57 & 0.0000 & & $\mathrm{RN}$ \\
\hline$\ldots$ & M31N 2009-11b & 7.9 & 0.38 & 10.56 & 0.0016 & $\mathrm{Fe}$ II & $\mathrm{RN}$ \\
\hline M31N 2008-12a & M31N 2009-12b & 1 & $\ldots$ & 48.92 & $\ldots$ & & $\mathrm{RN}$ \\
\hline$\ldots$ & M31N 2011-10e & 2.8 & 0.30 & 48.92 & 0.0001 & & $\mathrm{RN}$ \\
\hline$\ldots$ & M31N 2012-10a & 1.0 & 0.74 & 48.92 & 0.0002 & $\mathrm{He} / \mathrm{N}$ & $\mathrm{RN}$ \\
\hline$\ldots$ & M31N 2013-11f & 1.1 & 1.01 & 48.93 & 0.0005 & $\mathrm{He} / \mathrm{N}$ & $\mathrm{RN}$ \\
\hline
\end{tabular}

${ }^{a}$ M31N 1996-08c was discovered on 1997 August 01; not 1996 August 12 as reported in Shafter \& Irby 2001.

${ }^{\mathrm{b}}$ Coordinates for 2009-12b were not provided by Tang et al. (2014). 


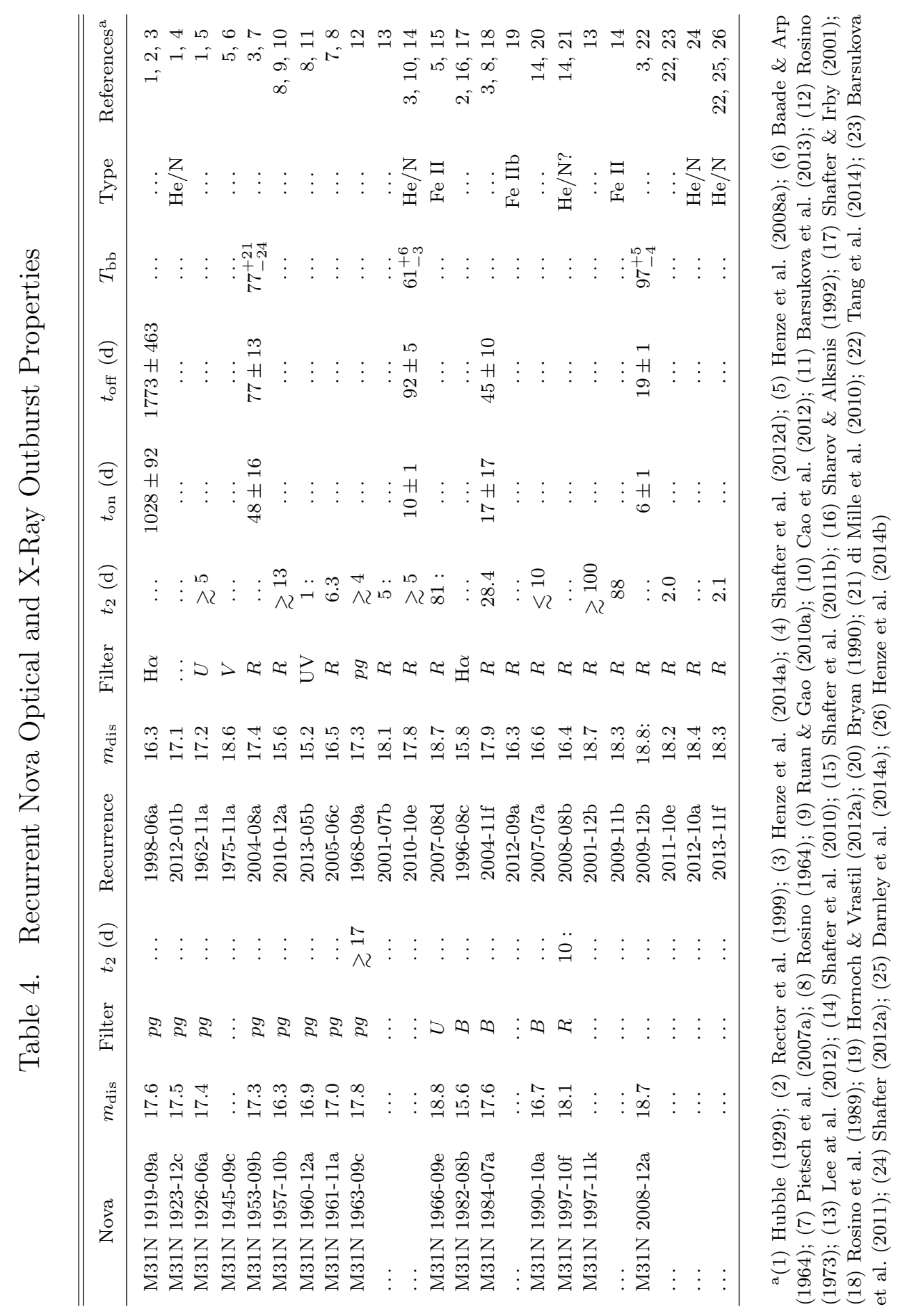


Table 5. Model Light Curve Properties

\begin{tabular}{|c|c|c|}
\hline Nova & $m_{B}(\max )$ & $\nu_{B}\left(\operatorname{mag~d}^{-1}\right)$ \\
\hline \multicolumn{3}{|c|}{ Classical Novae ${ }^{a}$} \\
\hline A04 & 18.2 & 0.200 \\
\hline A06 & 16.0 & 0.230 \\
\hline A07 & 15.9 & 0.150 \\
\hline $\mathrm{A} 12$ & 16.1 & 0.180 \\
\hline A13 & 17.0 & 0.077 \\
\hline A19 & 17.6 & 0.070 \\
\hline $\mathrm{A} 20$ & 17.2 & 0.060 \\
\hline A21 & 17.4 & 0.075 \\
\hline $\mathrm{A} 24$ & 17.8 & 0.059 \\
\hline $\mathrm{A} 25$ & 17.6 & 0.061 \\
\hline $\mathrm{A} 26$ & 18.0 & 0.043 \\
\hline A 29 & 18.0 & 0.017 \\
\hline R06 & 16.5 & 0.140 \\
\hline $\mathrm{R} 12$ & 17.6 & 0.050 \\
\hline $\mathrm{R} 18$ & 17.1 & 0.090 \\
\hline $\mathrm{R} 20$ & 16.9 & 0.090 \\
\hline $\mathrm{R} 28$ & 14.9 & 0.250 \\
\hline R30 & 16.2 & 0.170 \\
\hline R38 & 16.9 & 0.110 \\
\hline $\mathrm{R} 43$ & 16.3 & 0.220 \\
\hline $\mathrm{R} 52$ & 16.4 & 0.125 \\
\hline R53 & 17.0 & 0.040 \\
\hline $\mathrm{R} 57$ & 15.0 & 0.220 \\
\hline $\mathrm{R} 67$ & 16.2 & 0.130 \\
\hline $\mathrm{R} 77$ & 15.9 & 0.220 \\
\hline $\mathrm{R} 80$ & 17.4 & 0.034 \\
\hline $\mathrm{R} 85$ & 15.7 & 0.120 \\
\hline \multicolumn{3}{|c|}{ Recurrent Novae ${ }^{\mathrm{b}}$} \\
\hline T Pyx & 17.6 & 0.055 \\
\hline IM Nor & 17.9 & 0.039 \\
\hline CI Aql & 17.0 & 0.087 \\
\hline V2487 Oph & 17.3 & 0.340 \\
\hline U Sco & 16.2 & 1.410 \\
\hline V394 CrA & 15.8 & 0.705 \\
\hline $\mathrm{T} \mathrm{CrB}$ & 17.2 & 0.500 \\
\hline RS Oph & $16.4^{\mathrm{c}}$ & 0.254 \\
\hline V745 Sco & 16.7 & 0.328 \\
\hline V3890 Sgr & 15.9 & 0.260 \\
\hline \multicolumn{3}{|c|}{${ }^{a}$ From Capaccioli et al. (1989). } \\
\hline \multicolumn{3}{|c|}{$\begin{array}{l}{ }^{\mathrm{b}} \text { From Schaefer }(2010) . \text { Apparent magni- } \\
\text { tudes at the distance of M31 have been com- } \\
\text { puted assuming }(m-M)_{o}=24.38 \text { (Freed- } \\
\text { man et al. } 2001) \text { and a foreground extinction } \\
\text { of } A_{B}=0.25 \text { (Schlegel et al. 1998). }\end{array}$} \\
\hline \multicolumn{3}{|c|}{$\begin{array}{l}{ }^{\mathrm{c}} \text { Peak brightness for RS Oph is based on the } \\
\text { distance }(d=1.4 \mathrm{kpc}) \text { and reddening }(E(B- \\
V)=0.7) \text { given in Darnley et al. }(2012)\end{array}$} \\
\hline
\end{tabular}


Table 6. Monte Carlo Results

\begin{tabular}{|c|c|c|c|c|c|c|c|}
\hline $\begin{array}{c}(1) \\
\text { Coverage }\end{array}$ & $\begin{array}{c}(2) \\
m_{\lim }\end{array}$ & $\begin{array}{c}(3) \\
\text { CN Fraction }\end{array}$ & $\begin{array}{c}(4) \\
\text { RN Fraction }\end{array}$ & $\begin{array}{c}(5) \\
\text { RN/CN Dis. Efficiency }\end{array}$ & $\begin{array}{c}(6) \\
N_{\text {out }}(\mathrm{RNe}) / N_{\text {out }}(\mathrm{CNe})\end{array}$ & $\mathrm{CN}\left(\mathrm{yr}^{-1}\right)$ & $\begin{array}{c}(8) \\
\mathrm{RN}\left(\mathrm{yr}^{-1}\right)\end{array}$ \\
\hline \multicolumn{8}{|c|}{$t_{\mathrm{rec}}=1-100 \mathrm{yr}$} \\
\hline Clump1 & 17 & 0.0415 & 0.0034 & 0.0825 & 0.485 & 44 & 21 \\
\hline$\ldots$ & 18 & 0.1335 & 0.0360 & 0.2665 & 0.150 & 57 & 8 \\
\hline$\ldots$ & 19 & 0.2457 & 0.0936 & 0.3825 & 0.105 & 59 & 6 \\
\hline Clump3 & 17 & 0.0547 & 0.0095 & 0.1736 & 0.230 & 53 & 12 \\
\hline$\ldots$ & 18 & 0.1532 & 0.0563 & 0.3680 & 0.109 & 59 & 6 \\
\hline$\ldots$ & 19 & 0.2586 & 0.1119 & 0.4328 & 0.092 & 60 & 5 \\
\hline Clump5 & 17 & 0.0670 & 0.0162 & 0.2427 & 0.165 & 56 & 9 \\
\hline$\ldots$ & 18 & 0.1702 & 0.0758 & 0.4459 & 0.090 & 60 & 5 \\
\hline$\ldots$ & 19 & 0.2721 & 0.1287 & 0.4732 & 0.085 & 60 & 5 \\
\hline \multicolumn{8}{|c|}{$t_{\mathrm{rec}}=5-100 \mathrm{yr}$} \\
\hline Clump1 & 17 & $\ldots$ & 0.0010 & 0.0244 & 1.640 & 25 & 40 \\
\hline$\ldots$ & 18 & $\ldots$ & 0.0167 & 0.1237 & 0.323 & 49 & 16 \\
\hline$\ldots$ & 19 & $\ldots$ & 0.0564 & 0.2311 & 0.173 & 55 & 10 \\
\hline Clump3 & 17 & $\ldots$ & 0.0032 & 0.0592 & 0.676 & 39 & 26 \\
\hline$\ldots$ & 18 & $\ldots$ & 0.0295 & 0.1926 & 0.208 & 54 & 11 \\
\hline$\ldots$ & 19 & $\ldots$ & 0.0704 & 0.2726 & 0.147 & 57 & 8 \\
\hline Clump5 & 17 & $\ldots$ & 0.0062 & 0.0931 & 0.430 & 45 & 20 \\
\hline$\ldots$ & 18 & $\ldots$ & 0.0428 & 0.2518 & 0.159 & 56 & 9 \\
\hline$\ldots$ & 19 & $\ldots$ & 0.0839 & 0.3083 & 0.130 & 58 & 7 \\
\hline
\end{tabular}

